\title{
SPHERICAL SCATTERING OF SUPERPOSITIONS OF LOCALIZED WAVES
}

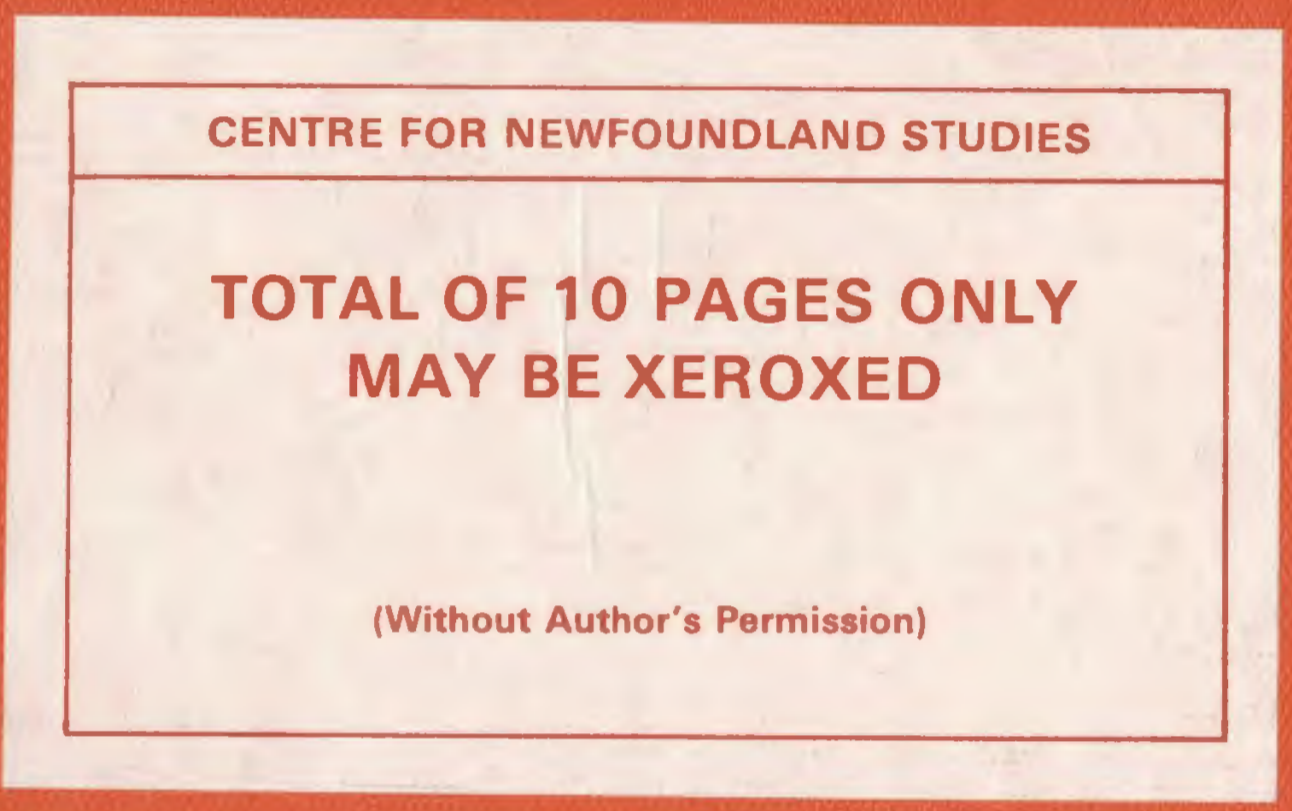

DESMOND POWER 


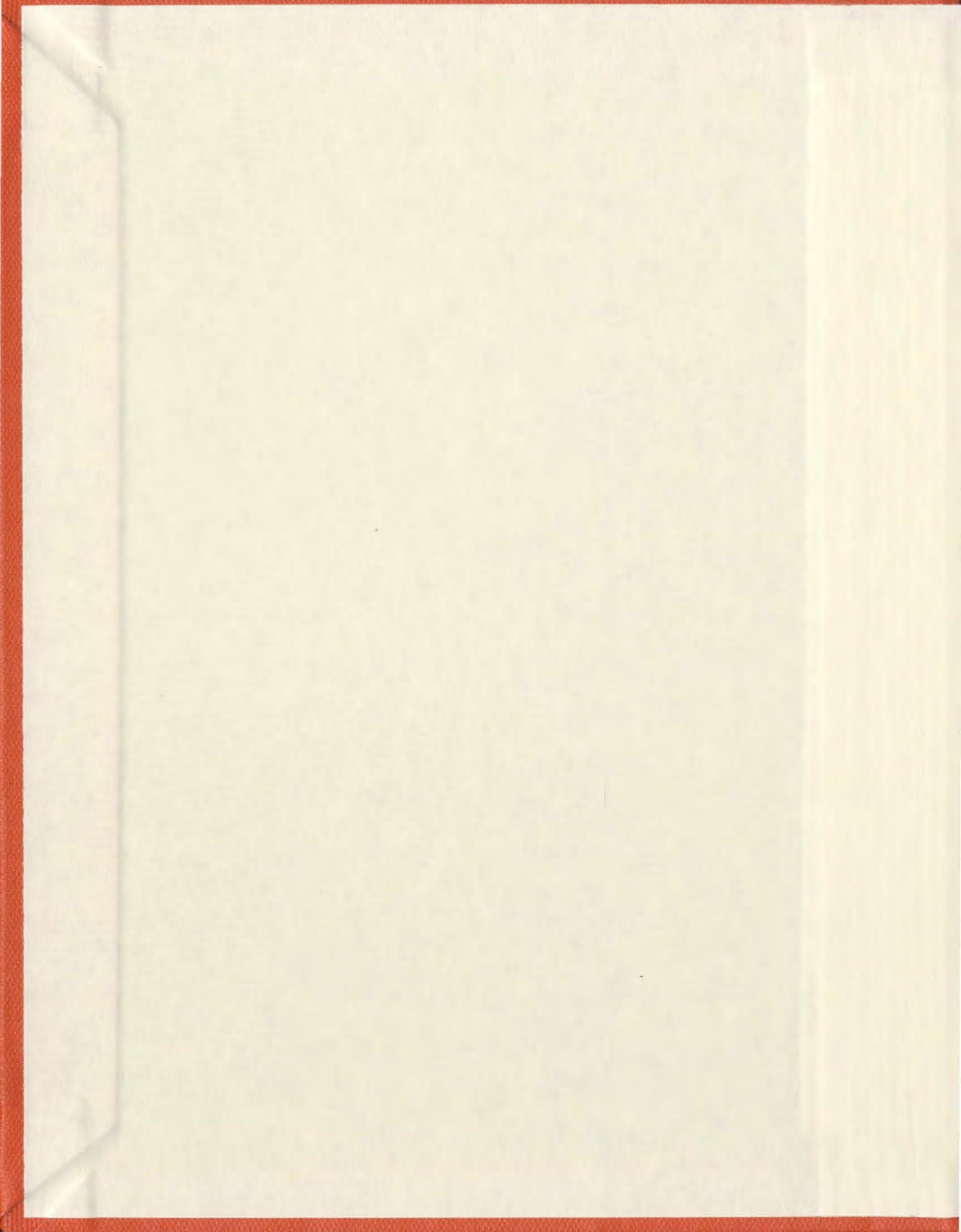




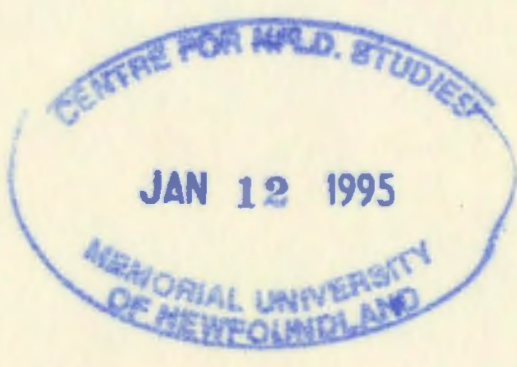




\title{
SPHERICAL SCATTERING OF SUPERPOSITIONS OF LOCALIZED WaVes
}

\author{
BY \\ - D Desmond Power, B.Eng.
}

A THESIS SUBMITTED TO THE SCHOOL OF GRADUATE STUDIES IN PARTIAL FULFILMENT OF THE REQUIREMENTS FOR THE DEGREE OF MASTER OF ENGINEERING

FACULTY OF ENGINEERING AND APPLIED SCIENCE MEMORIAL UNIVERSITY OF NEWFOUNDLAND ST. JOHN's, NEWFOUNDLAND, CANADA 
The author has granted an irrevocable non-exclusive licence allowing the National Library of Canada to reproduce, loan, distribute or sell copies of his/her thesis by any means and in any form or format, making this thesis available to interested persons.
L'auteur a accordé une licence irrévocable et non exclusive permettant à la Bibliothèque nationale du Canada de reproduire, prêter, distribuer ou vendre des copies de sa thèse de quelque manière et sous quelque forme que ce soit pour mettre des exemplaires de cette thèse à la disposition des personnes intéressées.

L'auteur conserve la propriété du droit d'auteur qui protège sa thèse. Ni la thèse ni des extraits substantiels de celle-ci ne doivent être imprimés ou autrement reproduits sans son autorisation. 


\section{Table of Contents}

List of Figures $\ldots \ldots \ldots \ldots \ldots \ldots \ldots \ldots \ldots \ldots \ldots$ ii

List of Tables ........................... . . . . . . .

Nomenclature . . . . . . . . . . . . . . . . . . . . iv

Abstract .............................. vii

Acknowledgements $\ldots \ldots \ldots \ldots \ldots \ldots \ldots \ldots \ldots \ldots \ldots \ldots \ldots \ldots$ viii

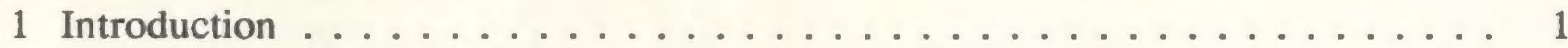

1.1 Preliminary Theory ... . . . . . . . . . . . . . 3

1.3.1 Hydrodynamics .................. 3

1.3.2 Wave Motion In A Compressible Fluid . . . . . . . . 4

2 Relevant Background ....................... 6

2.1 Focus Wave Modes ... . . . . . . . . . . . . . . 6

2.1 Designing Localized Waves ................. 7

2.2 Obtaining Finite Energy Solutions . . . . . . . . . . . 12

2.3 Launching a Localized Wave . . . . . . . . . . . . . . 16

2.4 Localized Wave Performance Bounds ... . . . . . . . . . . . 19

2.4.1 Radiated Field Efficiency . . . . . . . . . . . 22

2.4.2 Measured Field Efficiency . . . . . . . . . . . 26

2.4 .3 Beam Divergence . . . . . . . . . . . . . 27

2.5 Experimental Results . . . . . . . . . . . . . . . . . . . 29

2.5 .1 Simple Array . . . . . . . . . . . . . . . . . 29

2.5 .2 Folded Array . . . . . . . . . . . . . . 33

3 Predicted Backscatter Spectrum . . . . . . . . . . . . . . . . . . 36

3.1 Backscatter Spectrum Calculation . . . . . . . . . . . . . 36

3.2 Backscatter Spectrum Analysis ... . . . . . . . . . . 41

4 Experimental Methodology . . . . . . . . . . . . . . . . . . 45

4.1 Transmitter System . . . . . . . . . . . . . . . . . 45

4.2 Receiver System ..................... . . . 48

4.3 System Transfer Function . . . . . . . . . . . . . . . . . 49

4.4 Signal Reconstruction ... . . . . . . . . . . . . . . 50

4.5 Direct Signal Cancellation ................. 51

4.6 Background Noise . . . . . . . . . . . . . . . . 52

5 Experimental Results $\ldots \ldots \ldots \ldots \ldots \ldots \ldots \ldots \ldots \ldots$

6 Qualitative Analysis of Two Ball Scattering ... . . . . . . . . . . . 59

Conclusions . . . . . . . . . . . . . . . . . . . . . . . 62

References ... . . . . . . . . . . . . . . . . . . . . . 63 


\section{List of Figures}

Figure 2.1: The surface $\kappa^{2}+k_{x}^{2}-(\omega / c)^{2}$.

Figure 2.2: Projection of the support line of $\Psi_{\mathrm{FWM}}(\mathbf{k}, \omega)$, as given in (2.8) onto the $k_{z}, \omega / c$ plane (projection shown as solid line segment).

Figure 2.3: Projection of the support line of $\Psi_{\text {SUPER }}(k, \omega)$, as given in (2.13) onto the $k_{z}, \omega / c$ plane (projection shown as solid line segment). . . . . . . 11

Figure 2.4: MPS pulse, centre at $0 \mathrm{~m} \ldots \ldots \ldots \ldots \ldots \ldots \ldots$

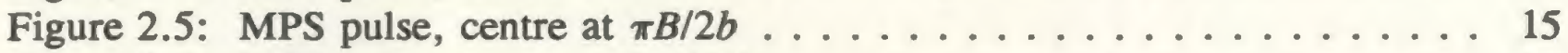

Figure 2.6: MPS pulse, centre at $3 \pi B / 4 b \ldots \ldots \ldots \ldots \ldots \ldots \ldots . \ldots \ldots$

Figure 2.7: MPS pulse, centre at $301 \pi B / 4 b . \ldots \ldots \ldots \ldots \ldots \ldots$

Figure 2.8: Hemisphere of radius $r_{1}$ on the $z=0$ plane projecting into $z>0$

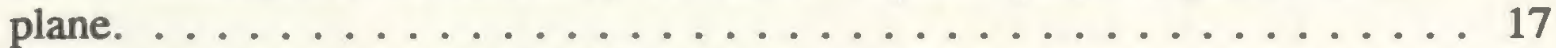

Figure 2.9: Transducer array with disk type elements. . . . . . . . . . 20

Figure 2.10: Disk transducer system response for $k_{\max } l \leq 1$. . . . . . . 20

Figure 2.11: Disk transducer system response for $k_{\max } l>1 \ldots \ldots \ldots \ldots$

Figure 2.12: (a) Piston and (b) Gaussian beam. . . . . . . . . . . . . . . . 29

Figure 2.13: Elements are folded onto the aperture (left) to produce a folded array (right). . . . . . . . . . . . . . . 33

Figure 3.1: Symmetry relationship for scattering expression in (3.3). . . . . 36

Figure 3.2: Projection of the support line of $\Psi_{\mathrm{FWM}}(\mathbf{k}, \omega, \beta)$, as given in (3.7) onto the $k_{z}, \omega / c$ plane (projection shown as solid line segment). . . . . . . 38

Figure 3.3: Surface plot of predicted backscattered spectra for varying sphere radius.

Figure 3.4: $\left|\Lambda_{\mathrm{MPS}}(r, \omega)\right|$ for $r=150 \mathrm{~mm}, c_{e}=3000 \mathrm{~m} / \mathrm{s}$, and $\varrho_{e}=7.8 \times 10^{3} \mathrm{~kg} / \mathrm{m}^{3}$ : $a=$ [50 (solid), 40 (dashed), and 30 (dotted)] $\mathrm{mm}$.

Figure 3.5: Surface plot of predicted backscattered spectra for varying sphere density.

Figure 3.6: Surface plot of predicted backscattered spectra for varying $c_{e} \ldots \ldots 43$

Figure 3.7: Surface plot of predicted backscattered spectra at $50 \mathrm{kHz}$ for varying

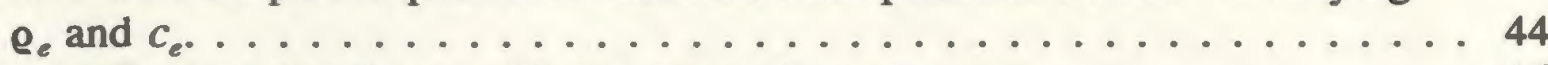

Figure 4.1: Backscatter Spectra Measurement System. . . . . . . . . . . 46

Figure 4.2: Sphere placed along the $z$-axis of the aperture. . . . . . . . 47

Figure 4.3: Frequency response of B\&K 8103 measured in water $1 \mathrm{~m}$ away from the hydrophone $[$ Brüel and Kjar, 1986] . . . . . . . . . . . . 48

Figure 4.4: Frequency response of Reson TC-4104 [Reson System APS, 1988]

Figure 4.5: Magnitude Plot of the double hydrophone system transfer function, $H(\omega)$ (solid), and a least squares fitted second order polynomial, $K \omega^{2}$ (dotted).

Figure 4.6: Hydrophone system response. . . . . . . . . . . . . . . . . 50

Figure 4.7: Typical residual signal (dotted) superimposed over the residual + spherically reflected signal. Notice that the difference between the two signals is noticable (barely) only after approximately $0.06 \mathrm{~ms}$. 
Figure 4.8: Spherically reflected signal obtained after the residual direct signal has been subtracted out. ..................... 52

Figure 4.9: FFT of received background after 10 averages (dotted) and after 50 averages (solid) . . . . . . . . . . . . . . . . . 53

Figure 5.1: Comparison of $\left|\Upsilon_{\text {MPS }}\right|$ (dotted) with $\left|H \Lambda_{\text {MPs }}\right|$ (solid) for a $30 \mathrm{~mm}$ radius steel sphere at $r=150 \mathrm{~mm}$ from the array. . . . . . . . . . . 54

Figure 5.2: Comparison of $\left|\Upsilon_{\text {MPs }}\right|$ (dotted) with $\left|H \Lambda_{\text {MPs }}\right|$ (solid) for a $40 \mathrm{~mm}$ radius steel sphere at $r=150 \mathrm{~mm}$ from the array. . . . . . . . . . . 54

Figure 5.3: Comparison of $\left|\Upsilon_{\text {MPS }}\right|$ (dotted) with $\left|H \Lambda_{\text {MPS }}\right|$ (solid) for a $60 \mathrm{~mm}$ radius steel sphere at $r=150 \mathrm{~mm}$ from the array.

Figure 5.4: Comparison of $\left|\Upsilon_{\text {MPs }}\right|$ (dotted) with $\left|H \Lambda_{\text {MPS }}\right|$ (solid) for a $40 \mathrm{~mm}$ radius aluminum sphere at $r=150 \mathrm{~mm}$ from the array. . . . . . . . .

Figure 5.5: Average spectral spacing plot for steel spheres. Graph shows both extracted (solid) and modelled (dashed) data. . . . . . . . . . . . . 56

Figure 5.6: Average spectral spacing plot for aluminum spheres. Graph shows both extracted (solid) and modelled (dashed) data. . . . . . . . . . . . 57

Figure 6.1: Placement of two spheres in from of acoustic array. . . . . . . . . 59

Figure 6.2: Two spheres, whose centres are separated by distance $d$, are placed at distance $r$ from the array. . . . . . . . . . . . . 59

Figure 6.3: Two ball backscatter spectrum for $r=25 \mathrm{~cm}$ and $d=4 \mathrm{~cm}$. . . . . 60

Figure 6.4: Two ball backscatter spectrum for $r=25 \mathrm{~cm}$ and $d=8 \mathrm{~cm} . \ldots \ldots 60$

Figure 6.5: Two ball backscatter spectrum for $r=25 \mathrm{~cm}$ and $d=14 \mathrm{~cm} . \ldots \ldots 60$

Figure 6.6: Two ball backscatter spectrum for $r=25 \mathrm{~cm}$ and $d=20 \mathrm{~cm}$. . . . . 60

Figure C.1: $\left|\Upsilon_{\text {MPS }}\right|$ for a $60 \mathrm{~mm}$ radius steel sphere at $r=250 \mathrm{~mm}$ from the array. ................................. 82

Figure C.2: $\left|\Upsilon_{\text {MPS }}\right|$ for a $40 \mathrm{~mm}$ radius steel sphere at $r=250 \mathrm{~mm}$ from the

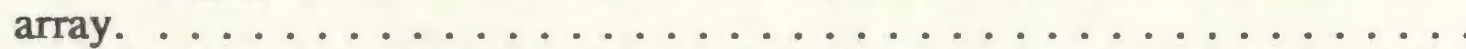

Figure C.3: $\left|\mathrm{T}_{\text {MPS }}\right|$ for a $30 \mathrm{~mm}$ radius steel sphere at $r=250 \mathrm{~mm}$ from the

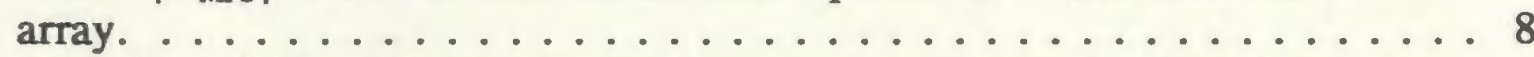

Figure C.4: $\left|\Upsilon_{\mathrm{MPS}}\right|$ for a $40 \mathrm{~mm}$ radius aluminum sphere at $r=250 \mathrm{~mm}$ from the

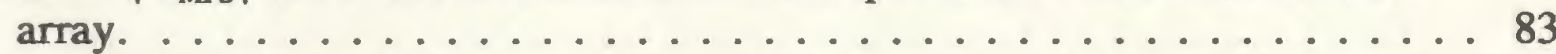

Figure C.5: $\left|\Upsilon_{\text {MPS }}\right|$ for a $60 \mathrm{~mm}$ radius steel sphere at $r=350 \mathrm{~mm}$ from the

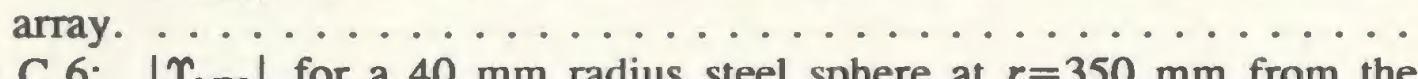

Figure C.6: $\left|\Upsilon_{\text {MPS }}\right|$ for a $40 \mathrm{~mm}$ radius steel sphere at $r=350 \mathrm{~mm}$ from the

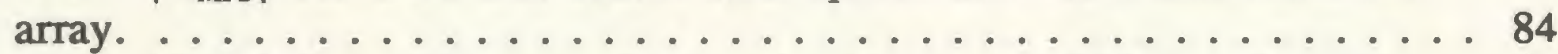

Figure C.7: $\left|\Upsilon_{\text {MPs }}\right|$ for a $30 \mathrm{~mm}$ radius steel sphere at $r=350 \mathrm{~mm}$ from the array. ................................ 85

Figure C.8: $\left|\Upsilon_{\text {MPS }}\right|$ for a $40 \mathrm{~mm}$ radius aluminum sphere at $r=350 \mathrm{~mm}$ from the array. . . . . . . . . . . . . . . . . . . 85

Figure C.9: Two ball backscatter spectrum for $r=35 \mathrm{~cm}$ and $d=4 \mathrm{~cm} . \ldots \ldots 86$

Figure C.10: Two ball backscatter spectrum for $r=35 \mathrm{~cm}$ and $d=8 \mathrm{~cm} . \ldots . .86$

Figure C.11: Two ball backscatter spectrum for $r=35 \mathrm{~cm}$ and $d=14 \mathrm{~cm}$. . . . 87

Figure C.12: Two ball backscatter spectrum for $r=35 \mathrm{~cm}$ and $d=20 \mathrm{~cm}$. . . . 87 


\section{List of Tables}

Table I: Speed of Sound in Various Rigid Materials . . . . . . . . . . . . . 44

Table II: Actual Vs. Extracted Sphere Radius . . . . . . . . . . . . . 58

\section{Nomenclature}

The following list of terms appears throughout the body of this document. They are defined here approximately in the order in which they appear in the text:

\begin{tabular}{||l||l||}
\hline $\begin{array}{c}\text { Term, } \\
\text { Variable }\end{array}$ & \\
\hline \hline LW & Localized Wave \\
\hline MPS & Modified Power Spectrum Pulse \\
\hline FWM & Focus Wave Mode \\
\hline HWE & Homogeneous Wave Equation \\
\hline$L$ & Rayleigh distance, diffraction length \\
\hline$f$ & frequency in Hz \\
\hline$\lambda$ & wavelength, (also used as a free parameter) \\
\hline$R_{a}$ & aperture radius \\
\hline$A$ & aperture area \\
\hline$p$ & pressure \\
\hline$m$ & mass \\
\hline F & force vector \\
\hline$V$ & volume \\
\hline e & density \\
\hline $\mathbf{V}$ & velocity vector \\
\hline a & acceleration vector \\
\hline$S$ & closed surface \\
\hline
\end{tabular}




\begin{tabular}{|c|c|}
\hline $\begin{array}{l}\text { Term, } \\
\text { Variable }\end{array}$ & Definition \\
\hline $\mathbf{S}$ & vector normal to surface $S$ \\
\hline$c$ & speed of sound \\
\hline$\psi(\mathbf{r}, t)$ & space-time waveform that satisfies the HWE \\
\hline$\Psi(k, \omega)$ & three dimensional spatial and temporal Fourier transform of $\psi$ \\
\hline $\mathbf{r}$ & $\begin{array}{l}\text { spatial observation coordinate, } \mathbf{r}=(x, y, z) \text { (cartesian), } \mathbf{r}=(\rho, \phi, z) \\
\text { (cylindrical), } \mathbf{r}=(r, \theta, \phi) \text { (spherical) }\end{array}$ \\
\hline$r$ & magnitude of $\mathbf{r}, r=\sqrt{x^{2}+y^{2}+z^{2}}$ \\
\hline $\mathbf{r}^{\prime}$ & spatial source coordinate \\
\hline$r^{\prime}$ & magnitude of $\mathbf{r}^{\prime}$ \\
\hline$t$ & time \\
\hline k & $\begin{array}{l}\text { transform variable corresponding to the spatial vector } \mathbf{r} \\
\mathbf{k}=\left(k_{x}, k_{y}, k_{z}\right) \text { (cartesian), } \mathbf{k}=\left(\kappa, \varphi, k_{z}\right) \text { (cylindrical), } \mathbf{k}=(r, \vartheta, \varphi) \\
\text { (spherical) }\end{array}$ \\
\hline$k$ & wavenumber, magnitude of $\mathrm{k}, k=\sqrt{k_{x}^{2}+k_{y}^{2}+k_{z}^{2}}$ \\
\hline$\omega$ & $\begin{array}{l}\text { frequency in radians/second, spatial transform variable } \\
\text { corresponding to } t\end{array}$ \\
\hline$\Xi$ & a weighting function \\
\hline $\operatorname{sgn}(x)$ & signum function, $\operatorname{sgn}(x)= \begin{cases}1 & x<0 \\
0 & x=0 \\
1 & x>0\end{cases}$ \\
\hline $\begin{array}{l}\beta, z_{0}, \gamma, B \\
b, \tau, T\end{array}$ & free parameters \\
\hline$J$ & Bessel function \\
\hline $\boldsymbol{H}$ & Hankel function \\
\hline$P$ & Legrendre polynomial \\
\hline$\underline{j}$ & spherical Bessel function \\
\hline$h$ & spherical Hankel function \\
\hline
\end{tabular}




\begin{tabular}{|c|c|}
\hline $\begin{array}{l}\text { Term, } \\
\text { Variable }\end{array}$ & Definition \\
\hline$g\left(\mathbf{r}, \mathbf{r}^{\prime}\right)$ & Propagator function, $1 / 4 \pi R$ \\
\hline $\boldsymbol{I}$ & field intensity \\
\hline$\Im$ & field fluence \\
\hline $\mathscr{E}$ & total energy, field energy \\
\hline$\underset{\rho}{\mathrm{HWHMM}}$ & half waist at half maximum \\
\hline$w$ & pulse waist \\
\hline$W$ & window function \\
\hline$\Gamma$ & gamma function, array efficiency \\
\hline $\begin{array}{l}\omega_{\text {rad }}, \Omega_{\text {rad }}, \\
\omega_{\text {m-as }}, \Omega_{\text {meas }}, \\
f_{\text {eff }}\end{array}$ & defined effective frequencies \\
\hline$\theta$ & beam divergence (also used in spherical coordinate system) \\
\hline$\alpha_{n}, \gamma_{n}$ & variables defined to simplify plane wave scattering expression \\
\hline$\delta$ & delta function \\
\hline$\Lambda$ & $\begin{array}{l}\text { spherical backscatter spectrum (temporal Fourier transform of signal } \\
\text { backscattered from a sphere) }\end{array}$ \\
\hline$u$ & unit step function \\
\hline$\kappa$ & compressibility, cylindrical spatial Fourier transform coordinate \\
\hline$v$ & reconstructed backscatter signal \\
\hline$\Upsilon$ & reconstructed backscatter spectrum, spatial Fourier transform of $v$ \\
\hline$\varsigma_{n}$ & backscatter signal from $n^{\text {th }}$ transmit element \\
\hline
\end{tabular}




\section{Abstract}

Localized wave (LW) solutions to the homogeneous wave equation represent broadband focused interference patterns whose focusing properties have been demonstrated to be superior to those of equivalent monochromatic (i.e. CW) solutions, in the context of multi-time-derivative transmit-receive systems. The focused, broadband nature of these pulses make them ideal candidates for use in remote sensing. The broadband nature of these LWs allows for enhanced target parameter extraction while the superior focusing properties ensure that a relatively large amount of energy reaches the target.

The spherically backscattered spectra of acoustic realizations of LWs are investigated for purposes of remote sensing. The LWs are acoustically launched with a synthetic hydrophone array. The backscattered spectra of several different sized steel and aluminum spheres have been obtained. Analysis indicates that the sphere radius can be readily extracted from these spectra. The backscattered spectra of steel sphere pairs have also been obtained. Preliminary analysis indicates that the single-sphere and double-sphere backscattered spectra have similar characteristics. However, it is questionable whether or not analysis of multi-sphere backscattered spectra will produce useful experimental data for a practical LW remote sensing system. 


\section{Acknowledgements}

I wish to thank sincerely my thesis supervisor, Dr. Rod Donnelly, for his important contribution to this work. It is through his patience, understanding and advice that this work stands finished. Thanks are also due to Dr. Richard Ziolkowski of the University of Arizona. His original research and experiments paved the way to the success of this project. I also thank him for his patience in replying to my endless Email questions.

I am deeply indebted to the Canadian Centre for Marine Communications (CCMC) for the extended use of their acoustic tank and equipment at the Marine Institute, St. John's, Newfoundland. I also thank Bob MacIsaac, who was the initial contact person with CCMC. Bob also provided very helpful advice in the use of CCMC's hydrophones and equipment. I also thank Brian McGraw of CCMC for his technical assistance, and Leo Spurrell and Humphrey Dye of Memorial University's Technical Services for the machined spheres.

Finally, I would like to thank my wife Brenda for her patience and understanding throughout the duration of my graduate studies. Brenda put up with my many late nights and weekends at the Marine Institute and the University, and I thank her for her kindness and love. 


\section{Introduction}

It is inherently true that all classical aperture launched space-time waveforms diffract as they propagate. This diffraction causes the maximum amplitude of the waveform to decay, generally with a $1 / r^{2}$ decrease in the field's energy as the energy spreads out over an increasing volume. Certain spatially localized energy beams have the ability to retain their localization before attaining the $1 / r^{2}$ energy decay. This phenomenon may be given in terms of a diffraction length or Rayleigh distance, $L_{R}$, which describes the distance before which a spatially localized beam attains a $1 / r^{2}$ decay. For example, a CW piston beam consisting of circular aperture uniformly driven with a continuous wave (CW) signal of frequency $f=c / \lambda$, will have natural focussing properties. For the CW piston with aperture radius $R_{a}$ and area $A=\pi R_{a}^{2}$, the diffraction length may be given by the classical Rayleigh distance [Ziolkowski, 1991]

$$
L_{\text {Pistou }}=\frac{A}{\lambda}=\frac{\pi R_{a}^{2}}{\lambda}
$$

Over the past few years, there has been great interest in space-time waveforms which have extended regions of localization, i.e. they have associated diffraction lengths which exceed classical Rayleigh distances. These waveforms, called Localized Waves (LWs), were first introduced by Brittingham [1983] and were studied in detail later by Ziolkowski $[1985,1988,1989,1990,1991]$. These waveforms do not defy the laws of physics since their physical realizations eventually attain a $1 / r^{2}$ energy decay. However, LWs have interesting propagation characteristics and their regions of extended localization have been demonstrated using acoustical transmit arrays in water. 
This work addresses possible uses of the LWs for remote sensing purposes. The broadband nature of the FWMs allow for the ability to extract target parameters. In order to test this possible application, backscatter experiments were performed on a number of steel and aluminum spheres using an acoustic LW realization.

In chapter two, the background theory of localized waves is discussed in a literature review. Since the fundamental $\mathrm{LW}$ is an infinite energy pulse, this chapter includes discussion on designing finite energy LWs from the fundamental infinite energy pulse. A particular finite energy LW called the Modified Power Spectrum Pulse (MPS) is described in detail. The results from Ziolkowski's [1989, 1990] localized wave experiments are also given. Using these results, Ziolkowski verified the superior propagation characteristics of LWs.

In chapter three, the predicted MPS spherical backscatter spectrum is calculated using techniques described in chapter two. The predicted backscattered spectrum is further analyzed to show a method of extracting the radius of the sphere. In chapter four, the methodology of obtaining the experimental backscattered spectrum is detailed. The acoustical launching array, along with the data acquisition system, is described in detail. In chapter five, the obtained spherical backscattered spectrum is analyzed. A computer algorithm is used to extract the radius of the sphere from the spectrum. In chapter six, the two ball experiments are described. In the next few sections, some relevant preliminary theory is presented for the purpose of completeness. 


\subsection{Preliminary Theory}

Since the main body of this work centres around acoustic scattering in a compressible fluid, it is necessary to discuss some fluid dynamics theory. The material which is presented may be found in a variety of texts including [Morse \& Ingard, 1968; Bowman, Senior \& Uslenghi, 1969; Wyld, 1976]. Ultimately, the discussion will lead to the derivation of the wave equation for a pressure field. The fact that a pressure field satisfies the wave equation has important repercussions for the research presented here. If one wishes to launch a particular pressure field, $p(r, t)$, then the equation describing the pressure field should be a solution to the wave equation.

\subsubsection{Hydrodynamics}

Consider first a small volume element of fluid, $d V$, to which Newton's second law may be applied:

$$
\mathbf{F}=m \mathbf{a}=\rho d V \frac{d v}{d t}
$$

In (1.2), $m=\varrho d V$ is the mass of the fluid element, $\varrho$ is its density, $a=d v / d t$ is its acceleration, and $\mathbf{v}$ is the velocity. In general, the density and velocity are functions of both space and time, $\varrho=\varrho(r, t), \mathbf{v}=\mathbf{v}(\mathbf{r}, t)$, thus the time derivative of the velocity may be written as

$$
\frac{d \mathbf{v}}{d t}=\frac{\partial \mathbf{v}}{\partial t}+\frac{\partial x}{\partial t} \frac{\partial \mathbf{v}}{\partial x}+\frac{\partial y}{\partial t} \frac{\partial \mathbf{v}}{\partial y}+\frac{\partial z}{\partial t} \frac{\partial \mathbf{v}}{\partial z}=\frac{\partial \mathbf{v}}{\partial t}+(\mathbf{v} \cdot \nabla) \mathbf{v}
$$

where $\nabla$ is the operator $(\partial / \partial x, \partial / \partial y, \partial / \partial z)$ in cartesian coordinates. The force, $\mathbf{F}$, and 
fluid pressure, $p$, may also be related through

$$
\mathbf{F}=-\nabla p d V
$$

Hence Newton's second law may be re-written as

$$
\varrho\left[\frac{\partial v}{\partial t}+(\mathbf{v} \cdot \nabla) \mathbf{v}\right]=-\nabla p
$$

In (1.5) it is assumed that the fluid is non-viscous and hence there is no energy loss by the forces of friction between molecules of the fluid.

We will also need the continuity equation for fluids:

$$
-\oint_{S} d \mathrm{~S} \cdot \mathrm{ev}=-\int_{V} d V \nabla \cdot\{\mathrm{ev}\}=\int_{V} d V \frac{\partial \mathrm{e}}{\partial t}
$$

where the volume integral on the RHS of (1.6) determines the rate of change of matter in volume $V$, while the surface integral on the LHS of (1.6) determines the flow of matter across the surface $S$ enclosing $V$. This can also be written in the form

$$
\frac{\partial \varrho}{\partial t}+\nabla \cdot\{Q v\}=0
$$

\subsubsection{Wave Motion In A Compressible Fluid}

In this section we will consider small oscillations (e.g. sound vibrations) in a compressible fluid of uniform average pressure and density, $p_{0} \& \varrho_{0}$. As such, the pressure and density may be denoted as $p=p_{0}+p^{\prime} \& \varrho=\varrho_{0}+\varrho^{\prime}$, where $p^{\prime} \& \varrho^{\prime}$ are the small oscillations in the medium pressure and density. If it is assumed that the pressure and density oscillations are small compared to their respective average values, then 
Newton's second law (1.5) can be approximated by

$$
\mathbf{e}_{0} \frac{\partial \mathbf{v}}{\partial t}=-\nabla p^{\prime}
$$

where the nonlinear terms $(v \cdot \nabla) v$ and $e^{\prime} v$ are assumed negligible. The continuity equation becomes

$$
\frac{\partial p^{\prime}}{\partial t}=\mathbf{e}_{0} \nabla \cdot \mathbf{v}
$$

If the pressure is assumed to be a function of density only, then the pressure and density fluctuations are related by the square of the speed of sound in the medium,

$$
p^{\prime}=c^{2} Q^{\prime}
$$

Hence, the homogenous wave equation may now be derived for the pressure fluctuations by taking the divergence of (1.8) and using (1.9) and (1.10) to eliminate $v$ and $\mathbf{e}^{\prime}$.

$$
\nabla^{2} p^{\prime}-\frac{1}{c^{2}} \frac{\partial^{2} p^{\prime}}{\partial t^{2}}=0
$$

In the next chapter, interesting localized wave solutions to the HWE will be discussed, along with their methods of design. Given an initial pressure field, $p$, one can easily determine the scattered field from objects of simple shapes. The research presented in chapters 3-6 focuses on the backscattered signal from spheres of arbitrary size and acoustical impedance. 


\section{Relevant Background}

Although this chapter is devoted to a literature review, it will be seen that a great portion of other authors' research is given here, rather than brief summaries of their work. This is deliberately done so that the reader may get a full picture of the objective of the new research presented later. It will be seen how naturally the previous research flows into the context of that presented in the chapters following.

\subsection{Focus Wave Modes}

Focus Wave Modes (FWMs) were first introduced by Brittingham in 1983. In his work, he described a family of three-dimensional, source free, non-diffracting pulses which propagate at the speed of light in free space. These FWMs are localized both along, and transverse to, the axis of propagation. Since the FWM is theoretically nondiffracting, the pulse envelope remains fixed as it propagates.

Brittingham's original work spurred on an number of papers related to the concept of transmitting focused energy beams. Of particular note is the work of Ziolkowski, who has investigated in depth the possibility of launching focused waves from a finite aperture [Ziolkowski, 1985, 1988, 1989, 1990, 1991]. Recent work by Donnelly \& Ziolkowski [1992, 1993] has detailed a framework for constructing localized waves (LWs). This review will concentrate on the FWM pulses, and mainly on the research listed above. 


\subsection{Designing Localized Waves}

Of particular importance to this research is a systematic method of constructing LW solutions. Such a method could be used to construct the original FWM as well as other LWs with different propagation characteristics. Donnelly \& Ziolkowski [1992, 1993] introduced such a method of obtaining solutions to constant coefficient homogeneous partial differential equations, such as the wave equation, the damped wave equation, and the Klein-Gordon equation, for example. This method is particularly important in the context of this thesis, and will now be described.

Consider the free space homogeneous wave equation (HWE),

$$
\left[\nabla^{2}-\frac{1}{c^{2}} \frac{\partial^{2}}{\partial t^{2}}\right] \psi(\mathbf{r}, t)=0
$$

A three dimensional spatial and temporal Fourier transform may be performed on (2.1) using the transform relationship

$$
\Psi(\mathbf{k}, \omega) \equiv \mathscr{F}_{r, t}\{\psi\}(\mathbf{k}, \omega)=\int_{\mathbf{R}} d \mathbf{r} \int_{-\infty}^{\infty} d t \psi(\mathbf{r}, t) e^{-i \mathbf{k} \cdot \mathbf{r}} e^{i \omega t}
$$

where $\mathbf{k}=\left(k_{x}, k_{y}, k_{z}\right) \equiv k_{x} \hat{x}+k_{y} y+k_{z} \hat{z}$, to give

$$
\left(k^{2}-\omega^{2} / c^{2}\right) \Psi(\mathbf{k}, \omega)=0 .
$$

One may readily design solutions to (2.3) using the generalized function relationship

$$
f(\mathbf{v}) \delta\left(\mathbf{v}-\mathbf{v}_{0}\right)=f\left(\mathbf{v}_{0}\right) \delta\left(\mathbf{v}-\mathrm{v}_{0}\right) \text {. }
$$

In this case, $\Psi$ may be designed using appropriate delta functions that force the function $\left(k^{2}-\omega^{2} / c^{2}\right)$ to become identically zero. 
In describing LWs, it is most convenient to use a cylindrical coordinate system. As such, (2.3) may be re-written as

$$
\left(\kappa^{2}+k_{z}^{2}-\omega^{2} / c^{2}\right) \Psi(\mathbf{k}, \omega)=0,
$$

where $\kappa=\sqrt{k_{x}^{2}+k_{y}^{2}}$ is the transform variable corresponding to the transverse coordinate $\rho$. Consider now the function

$$
\Xi(\kappa, \beta) \delta\left[k_{z}-f(\kappa, \beta)\right] \delta[\omega+c g(\kappa, \beta)] .
$$

If the functions $f$ and $g$ are set such that $\kappa^{2}+f^{2}(\kappa, \beta)-g^{2}(\kappa, \beta)=0$, then the delta functions will force $\left(k^{2}-\omega^{2} / c^{2}\right)$ to be identically zero. As such, the function (2.6) will be a solution of (2.5). The choice of the weighting function in (2.6) determines the amount of transverse localization. If the weighting function is set to be

$$
\Xi(\kappa, \beta)=\frac{\pi^{2}}{i \beta} e^{-\alpha^{2} 2 / 4 \beta},
$$

and the functions $f$ and $g$ are set to $\left(\beta-\kappa^{2} / 4 \beta\right)$ and $-\left(\beta+\alpha^{2} / 4 \beta\right)$ respectively, the resultant function is

$$
\Psi_{\mathrm{FWM}}(\mathrm{k}, \omega, \beta)=\frac{\pi^{2}}{i \beta} e^{-\kappa^{2} z_{j} / 4 \beta} \delta\left[k_{z}-\left(\beta-\kappa^{2} / 4 \beta\right)\right] \delta\left[\omega+c\left(\beta+\kappa^{2} / 4 \beta\right)\right] .
$$

The above equation is the three dimensional spatial and temporal Fourier transform of the FWM solution originally given by Ziolkowski [1985],

$$
\psi_{\mathrm{FWM}}(\mathbf{r}, t, \beta)=\mathscr{F}_{k, \omega}^{-1}\left\{\Psi_{\mathrm{FWM}}\right\}(\mathbf{r}, t)=e^{i \beta(z+c t)} \frac{e^{-\rho^{2} \beta /\left[z_{0}+i(z-c)\right]}}{4 \pi i\left[z_{0}+i(z-c t)\right]} .
$$

In $(2.7),(2.8)$ and $(2.9), \beta, z_{0}>0$ are free parameters. 
The method described above provides a means of controlling both the nonseparable nature and the localization of the designed solutions, $\psi$. The non-separability is determined by the delta function constraints, while the localization is determined through the weighting function, $\Xi$. The question which remains is how does one effectively manipulate the delta functions to produce solutions with desirable propagation characteristics.

Consider again the effect of the delta functions on equation (2.5). For example, the delta functions in equation (2.8) have the effect of forcing any of the transform variables $\left(\kappa, k_{z}, \omega\right)$ to lie on the surface

$$
\kappa^{2}+k_{z}^{2}-(\omega / c)^{2}=0 .
$$

This surface is illustrated in figure 2.1. The delta functions of (2.8) force the transform variables to lie on the line described by the two equations, $k_{2}=\beta$ $\kappa^{2} / 4 \beta$ and $\omega / c=-\left(\beta+\kappa^{2} / 4 \beta\right)$. The projection of this line onto the $k_{z}, \omega / c$ plane is given in figure 2.2 .

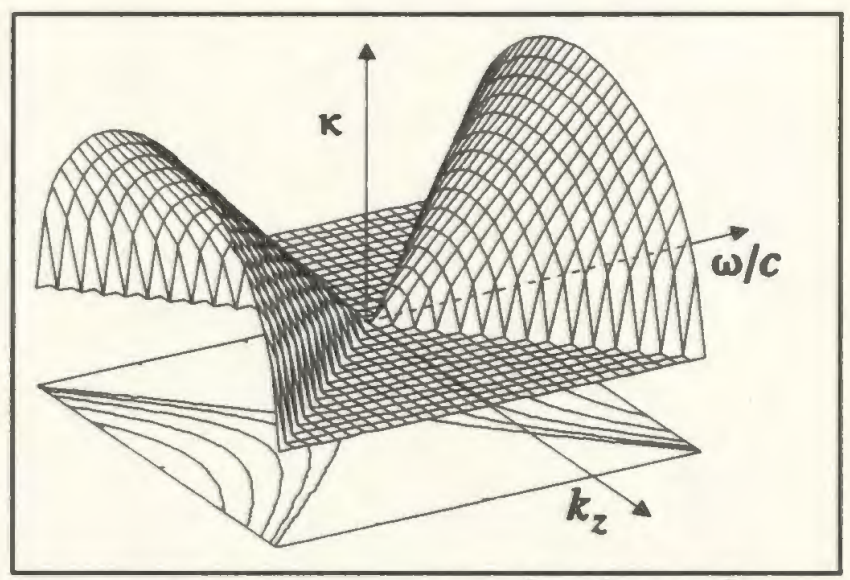

Figure 2.1: The surface $\kappa^{2}+k_{x}^{2}-(\omega / c)^{2}$.

When the free parameter, $\beta$, is set to 0 , the projection line of figure 2.2 coincides with the solution line given now by the two equations $\omega / c=k, k=0$. In this case there is no transverse localization and the $\psi_{\mathrm{FWM}}$ solution becomes a superposition of plane waves, each with frequency $\omega$ and speed $c$, travelling the $k_{z}$ direction. With this interpretation, Donnelly and Ziolkowski [1993] state that the FWMs represent the next 
generalization of HWE solutions from plane waves; the plane wave superposition support line is $\omega / c=k_{z}$, $x=0$, while the FWM support line is simply the parallel translation of the plane wave support line onto the surface of figure (2.1).

As shown further by Donnelly \& Ziolkowski [1993], the form of the LW

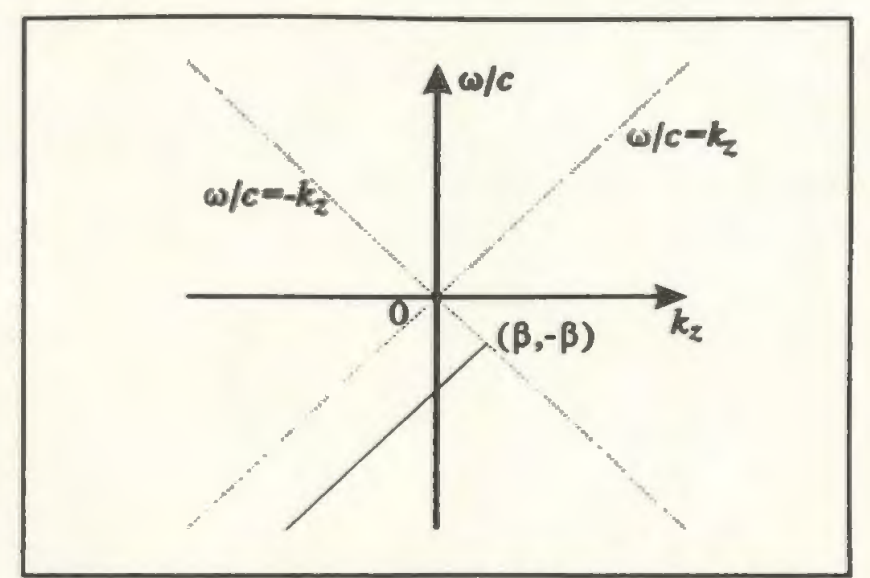

Figure 2.2: Projection of the support line of $\Psi_{\text {FWM }}(k, \omega)$, as given in (2.8) onto the $k_{z}$, $\omega / c$ plane (projection shown as solid line segment).

solutions is due not only to the support line, but also to the weighting function $\Xi$ along the line. Combinations of different support lines and weighting functions lead to LWs with enhanced localization properties and different propagation characteristics. For instance, if a different weighting function is chosen, such as

$$
\Xi(\kappa, \lambda, \beta)=\frac{z_{0} 4 \pi^{2}}{\beta} I_{0}\left(\frac{\lambda z_{0} \kappa}{2 \beta}\right) e^{-\lambda^{2} z_{0} / 4 \beta} e^{-2 \alpha_{0} \alpha^{2} / 4 \beta},
$$

with the same delta function support as the FWM solution, the solution becomes the zeroth order "Bessel-Gauss" pulse [Overfelt, 1991; Donnelly \& Ziolkowski, 1992; 1993],

$$
\begin{array}{r}
\psi_{\mathrm{BO}, 0}(\mathbf{r}, t, \beta, \lambda)=\frac{z_{0}}{z_{0}+i(z-c t)} J_{0}\left(\frac{\lambda z_{0} \rho}{z_{0}+i(z-c t)}\right) e^{i \beta(z+c n) e^{-\beta \rho^{2} /\left[z_{0}+i(z-c t)\right]}} \\
x e^{-i \lambda^{2} z_{0}(z-c n) / 4 \beta\left[z_{0}+i(z-c)\right]} .
\end{array}
$$

In (2.12) and (2.13) $\beta, z_{0} \& \gamma>0$ are free parameters. 
Now, if the solution support line on surface $(2.10)$ is changed such that the projection line on the $k_{2}$, $\omega / c$ plane becomes a straight line with slope $1 / \gamma$ emanating from the origin (figure 2.3), the Fourier transform of the solution is obtained, given by

$$
\Psi_{\text {SUPER }}(k, \omega, \gamma)=\Xi(\kappa, \gamma) \delta\left[k_{z}-\frac{\gamma \kappa}{\sqrt{1-\gamma^{2}}}\right] \delta\left(\omega-\frac{c \kappa}{\sqrt{1-\gamma^{2}}}\right) .
$$

Note that according to figure 2.3 the value of $\gamma$ should lie in the range $(-1,1)$ so that the projection line will correspond to a line on the surface of $(2.10)$. If the weighting function

$$
\Xi(\kappa, \gamma)=\frac{(2 \pi)^{3} \gamma^{2}}{1-\gamma^{2}} e^{-z_{0}|\gamma| \kappa(1-\gamma)^{2 n}}
$$

is now chosen, the resultant solution is

$$
\psi_{\text {SUPER }}(\mathbf{r}, t, \gamma)=\frac{\left[z_{0}-i \operatorname{sgn}(\gamma)(z-c t / \gamma)\right]}{\left\{\rho^{2}\left(1-\gamma^{2}\right) / \gamma^{2}+\left[z_{0}-i \operatorname{sgn}(\gamma)(z-c t / \gamma)\right]^{2}\right\}^{3 / 2}} .
$$

Since $\gamma$ lies in the range $(-1,1)$, it is easily seen that this solution represents a waveform travelling in the either the positive or negative $z$ direction with speed $|c / \gamma|>c$. In other words, the pulse is superluminal with speed depending on $\gamma$.

As illustrated by Donnelly \& Ziolkowski [1993], many other choices of the solution support and the weighting function can be made to form other interesting solutions to the HWE. The authors also extend the HWE methodology described above 
to the damped wave and the Klein-Gordon equations. In this review, emphasis will be placed on the FWM solution (2.8).

\subsection{Obtaining Finite Energy Solutions}

As shown by Ziolkowski [1985], the FWM solution given by (2.9) has finite energy density but infinite total energy. If the LW solutions are to be physically launched, it is desirable that they have finite energy. Finite energy LW solutions may be formed from the infinite energy FWM solutions using superposition. Consider that if a function $\psi(\beta)$ is a solution to the HWE, where $\beta$ is an arbitrary parameter, then a weighted superposition of the function $\psi(\beta)$, over the parameter $\beta$, will also be a solution the HWE. Using this reasoning, the superposition

$$
\int_{0}^{\infty} d \beta F(\beta) \psi_{\mathrm{FWM}}(\mathbf{r}, t, \beta)
$$

is also a solution to the HWE [Ziolkowski, 1985; Donnelly \& Ziolkowski 1992; 1993], where $F$ is an arbitrary weighting function. Ziolkowski [1985] first showed that the superposition in (2.16) will lead to a finite energy solution provided that

$$
\int_{0}^{\infty} d \beta \frac{|F(\beta)|}{\beta}<\infty .
$$

This constraint on $F$ will change for different LW solutions depending the choice of the delta function support and the weighting function $\Xi$ used to extract a LW solution. Donnelly \& Ziolkowski [1993] outline a method for determining these constraints. If the 
superposition of an arbitrary HWE solution $\psi$ is denoted by

$$
\Theta(\mathbf{r}, t)=\int_{0}^{\infty} d \beta F(\beta) \psi(\mathbf{r}, t, \beta)
$$

then the total energy of this solution may be given by

$$
\mathscr{E}=\int_{\mathbf{R}^{s}} d \mathbf{r}|\Theta(\mathbf{r}, t)|^{2}
$$

This expression can be shown [Donnelly \& Ziolkowski, 1992] to be re-written as

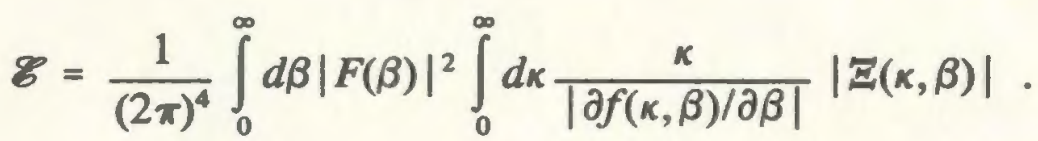

where the function $f$ was given in (2.6). If the integrals in (2.20) converge, then the superposition has produced a function $\theta$ with finite energy. In the case of the LW pulses, $f(\kappa, \beta)=\beta-\kappa^{2} / 4 \beta$ and $|\Xi(\kappa, \beta)|=e^{-\kappa^{2} z_{0} / 2 \beta}$, hence the total energy of the superposed $\mathrm{LW}$ is given by

$$
\mathscr{E}_{\mathrm{LW}}=\frac{1}{16} \int_{0}^{\infty} d \beta|F(\beta)| \int_{0}^{\infty} d \kappa \frac{\kappa}{\kappa^{2}+4 \beta^{2}} e^{-\alpha^{2} 2 / 2 \beta} .
$$

As shown in [Donnelly \& Ziolkowski, 1993], this integral will lead to the result previously given in (2.17) for finite total energy. 
For the general FWM superposition, a finite energy solution may be written as

$$
\psi_{\mathrm{LW}}^{\mathrm{FNTE}}=\frac{1}{4 \pi i\left[z_{0}+i(z-c t)\right]} \int_{0}^{\infty} d \beta F(\beta) e^{-\beta s},
$$

where $s=\rho^{2} /\left[z_{0}+i(z-c t)\right]-i(z+c t)$, assuming the energy criterion of (2.17) is met. Examination of (2.22) reveals that the integral is in the form of a Laplace transform [Ziolkowski, 1985], i.e.

$$
\psi_{\mathrm{LW}}^{\mathrm{FINITE}}=\frac{1}{4 \pi i\left[z_{0}+i(z-c t)\right]} \mathscr{L}_{\beta}\{F\}(s)
$$

Hence interesting finite energy LW solutions may be obtained by simply consulting a table of Laplace transforms. One such solution introduced by Ziolkowski [1985] is called the Modified Power Spectrum (MPS) pulse. Its name is derived from the power spectrum $F(\beta)=\beta^{(T-1)} e^{-n \beta}$ appropriately scaled and truncated to give the spectral weighting

$$
F(\beta)=\left\{\begin{array}{lr}
4 \pi i B(B \beta-b)^{T-1} \frac{e^{-r(B \beta-b)},}{\Gamma(T)}, & \beta>\frac{b}{B} \\
0, & 0 \leq \beta<\frac{b}{B}
\end{array}\right.
$$

The parameters, $\tau, b, T, B$ in the above expression are free parameters. Performing the superposition leads to the space-time expression for the MPS pulse,

$$
\psi_{\mathrm{MPS}}(\mathbf{r}, t)=\frac{1}{z_{0}+i(z-c t)} \frac{1}{(s / B+\tau)^{T}} e^{-b s / B}
$$

The MPS has some interesting propagation properties, as outlined in [Ziolkowski, 1985]. For instance, if $B \gg 1$ and $T=1$ in (2.25), the real part of $\Psi_{\text {MPs }}$ is given by 


$$
\begin{aligned}
& \psi_{\mathrm{MPS}}(\rho=0, z=c t)=\frac{\cos (2 b z / B)-(2 z / B \tau) \sin (2 b z / B)}{1+(2 z / B \tau)^{2}} \frac{1}{\tau z_{0}} \\
& \approx \begin{array}{ll}
1 / \tau z_{0} & \text { when } 2 z / B \tau<1 \\
\cos (2 b z / B) / \tau z_{0} & \text { and } 2 b z / B<1 \\
{\left[\sin (2 b z / B) /\left(2 z_{0} / B\right)\right](1 / z)} & \text { when } 2 z / B \tau<1
\end{array} \\
&
\end{aligned}
$$

From (2.26) it can be seen that the MPS pulse centre retains a contant amplitude whe $2 b z / B<1$ and $2 z / B \tau<1$. The pulse centre then begins to oscillate with period $\pi B / b$ in the region $B / 2 b<z<B \tau / 2$, and begins to decay in the region $z>B \tau / 2$. Figures 2.4-2.7 show plots of $\operatorname{Re}\left\{\psi_{\mathrm{MPs}}\right\}$ for the various regions given above, using the parameters $z_{0}=0.004$ $\mathrm{m}, T=1, \tau=1.0 \mathrm{~m}, B=300$ and $b=750 \mathrm{~m}^{-1}$.

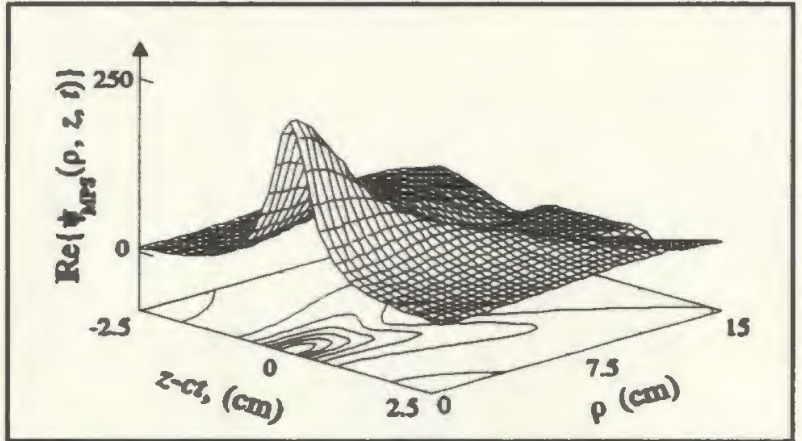

Figure 2.4: MPS pulse, centre at $0 \mathrm{~m}$

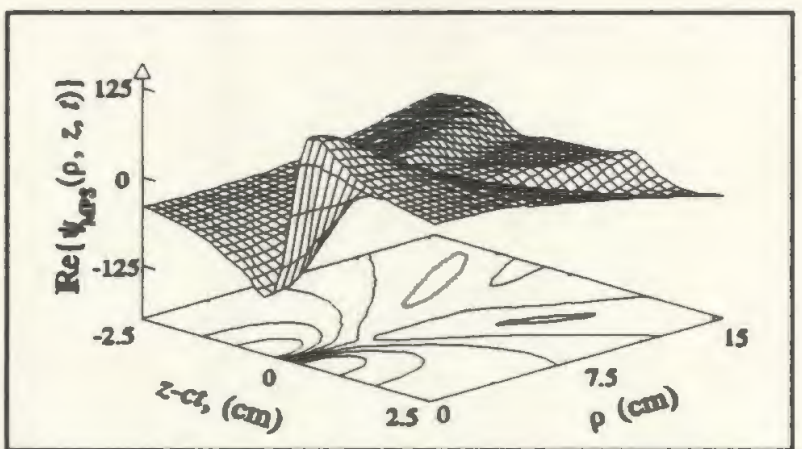

Figure 2.6: MPS pulse, centre at $3 \pi B / 4 b$.

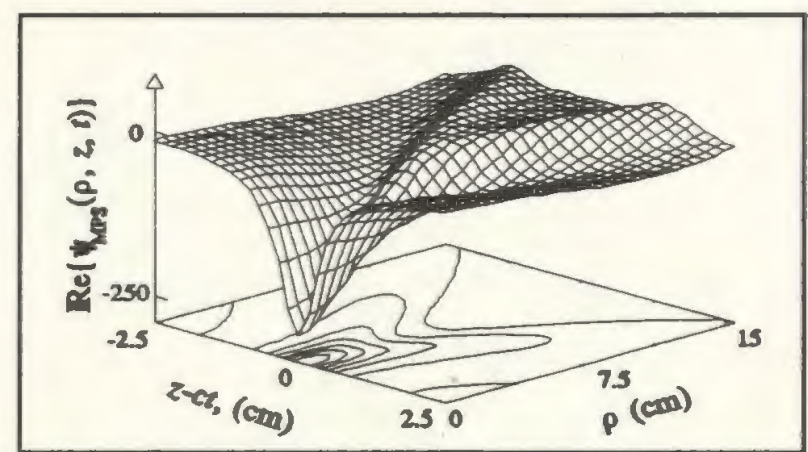

Figure 2.5: MPS pulse, centre at $\pi B / 2 b$

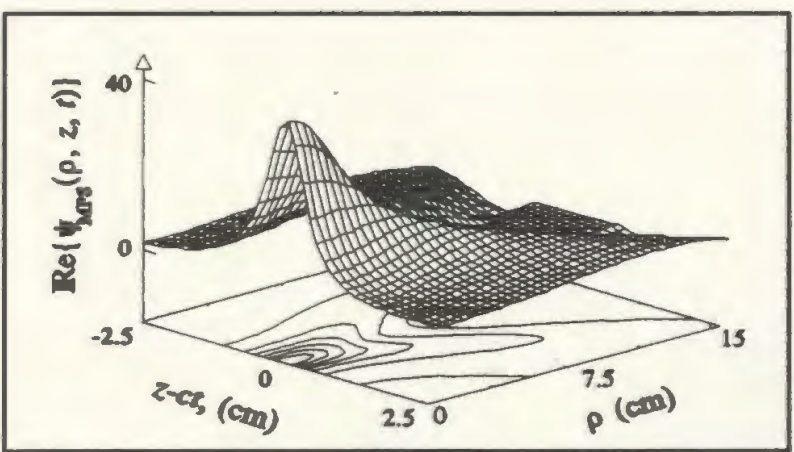

Figure 2.7: MPS pulse, centre at $301 \pi B / 4 b$. 


\subsection{Launching a Localized Wave}

Several successful attempts have been made to launch approximations to LW pulses [Ziolkowski, 1985; 1991; Ziolkowski \& Lewis, 1989; 1990; Donnelly et al, 1994], both in numerical simulations and in acoustic realizations. The realization of these pulses is made possible through the use of Huygens' representation [Jones 1964],

$$
\begin{gathered}
\psi(\mathbf{r}, t)=\oint_{S}\left\{g\left(\mathbf{r}, \mathbf{r}^{\prime}\right) \partial_{n^{\prime}}\left[\psi\left(\mathbf{r}^{\prime}, t-R / c\right)\right]-\psi\left(\mathbf{r}^{\prime}, t-R / c\right)\left[\partial_{n^{\prime}} g\left(\mathbf{r}, \mathbf{r}^{\prime}\right)\right]\right. \\
\left.+g\left(\mathbf{r}, \mathbf{r}^{\prime}\right) \partial_{c l}\left[\psi\left(\mathbf{r}^{\prime}, t-R / c\right)\right] \partial_{n^{\prime}} R\right\} d S^{\prime}
\end{gathered}
$$

where $\psi$ is a scalar field satisfying the HWE, $S$ is a closed surface ( $\mathrm{r}$ lies inside $S$ ), $n^{\prime}$ is the inward pointing normal to $S, R=\sqrt{\left(x-x^{\prime}\right)^{2}+\left(y-y^{\prime}\right)^{2}+\left(z-z^{\prime}\right)^{2}}$ is the distance between the source coordinates $\left(x^{\prime}, y^{\prime}, z^{\prime}\right)$ on $S$ and the observation coordinates $(x, y, z)$ contained within $S$, and $g\left(r, r^{\prime}\right)=1 / 4 \pi R$. This equation can be interpreted as follows: the HWE solution scalar field $\psi$ inside any closed volume $S$ can be reconstructed by simply knowing the normal derivative of $\psi$, the time derivative of $\psi$ and the value of $\psi$ on the surface $S$. Hence if the surface $S$ is excited with the function given inside the integrand of (2.27), $\psi$ will be exactly reconstructed at the point $\mathbf{r}$ for all time. Using this interpretation of (2.27), it is possible, with a few approximations, to launch a facsimile of a scalar field LW into a region of space.

Consider first $S$ being the surface consisting of a circle on the plane $z=0$ and the top half of a spherical shell, the half pointing towards positive $z$ (figure 2.8). Both the sphere and the circle are centred at the origin and both have radius $r_{1}$. If $r_{1} \rightarrow \infty$, the surface $S$ encloses the region $z>0$ and the reconstruction point $\mathbf{r}$ is contained in the region $z>0$. Since the LW pulses have most of their energy contained within a small 
region about the axis of propagation, an approximation to the reconstruction of $\psi$ at $\mathbf{r}$ can be made by exciting a small aperture on the surface $S$ centred at the origin. This is essentially Kirchhoff's approximation [Jones, 1964]. As such,

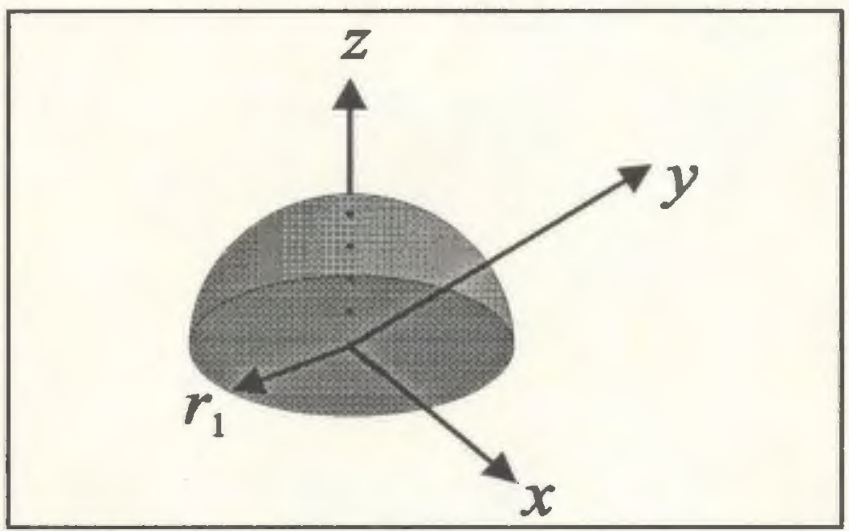

Figure 2.8: Hemisphere of radius $r_{1}$ on the $z=0$ plane projecting into $z>0$ plane.

(2.27) may be re-written as

$$
\psi(\mathbf{r}, t)=-\int_{A} d S^{\prime} \Phi\left(\mathbf{r}^{\prime}, t-R / c\right) \frac{1}{4 \pi R}
$$

where $\boldsymbol{A}$ is the area of the aperture on the plane $z^{\prime}=0$, and the driving function $\Phi$ is given by

$$
\Phi\left(\mathbf{r}^{\prime}, t-R / c\right)=\left[\partial_{z^{\prime}} \psi\right]-\left[\partial_{c t} \psi\right] \frac{Z}{R}-[\psi] \frac{z}{R^{2}}
$$

The square brackets in (2.29) indicate that the function inside is to be evaluated at the retarded time, $t-R / c$. Since the driving function $\Phi$ depends on the observation coordinate $\mathbf{r}$, it seems that it may be possible to reconstruct the $\mathrm{LW}$ only at a single point. However, if it is assumed that the reconstruction point is sufficiently far away from the aperture and close enough to the $z$-axis to assume that $R \approx z$, the driving function may be approximated by $\Phi \approx 2 \partial_{c s} \psi$. Hence a LW pulse may now be reconstructed by approximating $(2.28)$ by 


$$
\psi(\mathbf{r}, t) \approx \sum_{n=1}^{N} \Phi\left(\mathbf{r}_{\mathrm{n}}, t-R / c\right) \frac{A_{n}}{4 \pi R},
$$

where $N$ is the number of transducers placed uniformly on the aperture $A, \mathbf{r}_{n}$ is the discrete location of each transducer on the aperture, and $A_{n}$ is an area weighting for each element, replacing $d S^{\prime}$ in (2.28). For a uniform array of equally spaced elements, $A_{n}$ is constant for all $n$. The function $\Phi$ now becomes the driving function for point source transducers whose impulse response is modelled by

$$
h\left(\mathbf{r}, \mathbf{r}_{n}, t\right)=\delta(t-R / c) / 4 \pi R
$$

Equation (2.31) gives the impression that the radiation pattern of the point sources should be spherically symmetric. However, it is assumed that the observation point is far from the array and close to the $z$-axis. This means that the angle between the transducer's axis and the observation point will be small. This allows the use of certain transducers with directive radiation patterns. For instance, Ziolkowski [1991] uses disk transducers having radiation pattern described by $2 J_{1}(k a \sin \theta) /(k a \sin \theta)$ where $a$ is the radius of the disk and $k$ is the wavenumber of the sinusoid driving the disk. Since $\theta$ is assumed to be small when reconstructing near the $z$-axis and far from the array, the radiation pattern term may be set to unity. In using such an approximation, it is assumed that the sidelobe levels of the array are not adversely affected (i.e. they do not increase). 


\subsection{Localized Wave Performance Bounds}

In the previous sections, a method of designing and launching localized wave solutions, which are non-separable in space and time, has been discussed. It will be shown in this section that this non-separability provides for extended regions of localization over their CW counterparts.

In order to launch a LW, a finite array of point sources may be driven with the broadband LW signals given in (2.30). Conventionally, a focused pulse is produced by driving an array of point sources with a gated $\mathrm{CW}$ signal. The inherent differences between the signals launched make it difficult to compare their propagation characteristics and beam qualities. For instance, traditional performance criteria such as Rayleigh distance, beam spread, beam intensity and beam energy are based on narrowband concepts and are not readily applicable to the broadband case. However, in [Ziolkowski, 1991], performance bounds are derived based on the measurement of an effective frequency for the LW arrays. All of the theoretical analysis presented in this section is taken from [Ziolkowski, 1991]. Experimental data which supports this theoretical analysis can be found in [Ziolkowski, 1991; Ziolkowski \& Lewis, 1989; 1990]

Consider an array of $N$ independently addressable radiating elements placed on an aperture as illustrated in figure 2.9. Each element has radiating area $A_{n}$ and is excited with the driving function $\Phi_{n}(t)=\Phi\left(\mathrm{r}_{n}, t\right)$. The total area the array is $A=\sum_{n=1}^{N} A_{n}$. The experiments discussed in [Ziolkowski \& Lewis, 1989; 1990] employed small disk transducers which have the approximate transmit/receive characteristics illustrated in figures 2.10 and 2.11. Further analysis of these transducers may be found in many texts, 
including Ristic [1983].

In figure 2.10 , it is assumed that the characteristic length, $l$, of each element is not large in comparison with the shortest significant wavelength, $\lambda_{\min }$, driven into each element, or $2 \pi l / \lambda_{\min }=k_{\max } l \leq 1 . \quad$ A voltage signal driven into a transmitting transducer will experience a single time derivative from the electro-mechanical energy conversion. The signal then experiences a second time derivative from the radiation process, which converts energy near the transducer's face to energy far from the transducer. A third time derivative occurs from electro-mechanical energy conversion at the receiving transducer. In this case, the radiated field is defined as the field near the transducer face and is indicated in figure 2.10.

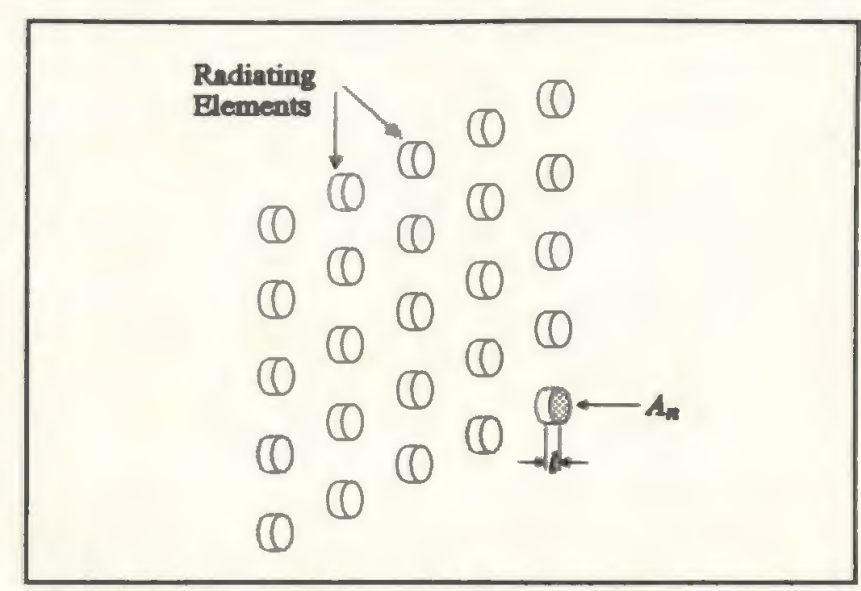

Figure 2.9: Transducer array with disk type elements.

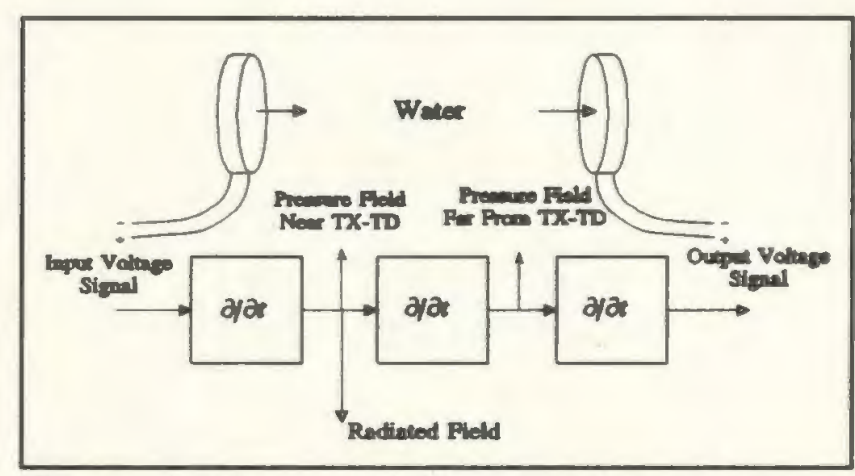

Figure 2.10: Disk transducer system response for $k_{\max } l \leqslant 1$.

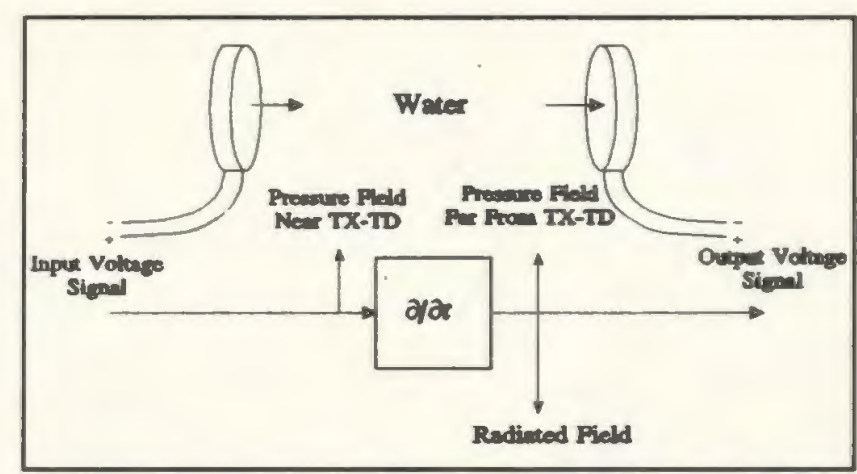

Figure 2.11: Disk transducer system response for $k_{\max } l \gg 1$.

Next, consider the case in which the array elements are large in comparison with the shortest significant wavelength, i.e. $k_{\max } l \gg 1$. In this case, the transducer's electro- 
mechanical conversion process does not have any effect on the signal; the system will impart a single time derivative on the signal due to the radiation process. In this case, the radiated field is defined as the field far from the transducer face and is indicated in figure 2.11.

For both the small and large transducer models, the input driving functions experience a single time derivative when being transmitted from the input to the radiated field. Then, according to the previous section, an approximate LW may be launched as the radiated field if the transducers are instead driven with the new set of functions, $\psi_{n}=$ $\psi\left(\mathbf{r}_{n}, t\right)$ (Note that the factor of two has been dropped). The driving fuctions will consitute the input field. The units of an input signal are taken to be $\left(\mathrm{W} / \mathrm{m}^{2}\right)^{1 / 2}$. The expression for the total radiated field may be approximated using (2.30) in conjunction with the radiated field model in figures 2.10 and 2.11 ,

$$
\psi_{\mathrm{rad}}(\mathbf{r}, t) \approx \sum_{n=1}^{N} \frac{A_{n}}{2 \pi c\left|\mathbf{r}-\mathbf{r}_{n}\right|} \partial_{\imath} \psi_{n}\left(\mathbf{r}_{n}, t-\left|\mathbf{r}-\mathbf{r}_{n}\right| / c\right)
$$

When the transducers are small, i.e. when $k_{\max } l \leq 1$, the radiated field is not the same as measured field, the field given at the output of the receiving transducers. The time derivatives caused by the radiation and measurement process should be taken into account (c.f. figure 2.10), hence

$$
\psi_{\text {meas }}(\mathbf{r}, t) \approx C \sum_{n=1}^{N} \frac{A_{n}}{2 \pi c\left|\mathbf{r}-\mathbf{r}_{n}\right|} \partial_{\varepsilon}^{3} \psi_{n}\left(\mathbf{r}_{n}, t-\left|\mathbf{r}-\mathbf{r}_{n}\right| / c\right)
$$

The constant $C$ is introduced by the conversion from radiated to measured fields as detailed in [Ziolkowski, 1991]. 
In order to evaluate the beam quality of the LWs, the quantities of input field energy, $\mathscr{E}$, beam intensity, $l$, and fluence, $\Im$, will now be defined:

$$
\begin{aligned}
& \mathscr{Z}_{\text {field }}=\int_{A_{\infty}} d S^{\prime} \int_{-\infty}^{\infty} d t\left|\psi_{\text {field }}(\mathbf{r}, t)\right|^{2}, \quad[\mathrm{~J}] \\
& I_{\text {field }}(\mathbf{r}, t)=\left|\psi_{\text {field }}(\mathbf{r}, t)\right|^{2}, \quad\left[\mathrm{~W} / \mathrm{m}^{2}\right] \\
& \Im_{\text {field }}(\mathbf{r})=\int_{-\infty}^{\infty} d t I_{\text {ficeld }}(\mathbf{r}, t) . \quad\left[\mathrm{J} / \mathrm{m}^{2}\right]
\end{aligned}
$$

These quantities are readily derivable for each of the array driven field quantities: input, radiated and measured.

\subsubsection{Radiated Field Efficiency}

In this section, the efficiency of the radiated fields of the LW and CW driven arrays will be compared. In this case, the efficiency of the array will be defined as the ratio of radiated to input field fluence,

$$
\Gamma_{\mathrm{rad}}=\frac{\Im_{\mathrm{rad}}}{\Im_{\mathrm{ind}}}
$$

The input and radiated field fluence can be calculated for the array of transducers

as, 


$$
\begin{gathered}
\Im_{\text {in }}=\frac{\mathscr{E}_{\text {in }}}{A}=\frac{1}{A}\left[\sum_{n=1}^{N} A_{n} \int_{-\infty}^{\infty} d t\left|\psi_{n}\left(\mathbf{r}_{n}, t\right)\right|^{2}\right) \\
\Im_{\mathrm{rad}}(\mathbf{r}) \leq\left(\frac{A}{2 \pi c z}\right)^{2} \frac{U_{\mathrm{rad}}}{A}
\end{gathered}
$$

where

$$
U_{\mathrm{rad}}=\sum_{n=1}^{N} U_{n}=\sum_{n=1}^{N} A_{n} \int_{-\infty}^{\infty} d t\left|\partial_{t} \psi_{n}\left(\mathbf{r}_{n}, t\right)\right|^{2} .
$$

First, consider the CW driven array. The array is uniformly spaced with each of the sources having the same area, i.e $A_{n}=A_{0}$ and $A=N A_{0}$. Each element is driven with the same CW signal having angular frequency $\omega_{\mathrm{Cw}}$ so that $\int d t\left|\partial_{\imath} \psi\right|^{2}=\omega_{\mathrm{CW}}^{2} \int d t|\psi|^{2}$, and the input energy is the same for each element, $\mathscr{E}_{n}^{i m}=\mathscr{Z}_{0}^{i n}$. The input fluence, radiated fluence and far field efficiency are given by

$$
\begin{gathered}
\Im_{\text {in }}^{C W}=\frac{\mathscr{E}_{0}^{\text {in }}}{A_{0}}, \\
\Im_{\text {red }}^{\mathrm{CW}} \leq\left(\frac{A}{2 \pi c z}\right)^{2} \frac{U_{0}}{A_{0}}, \\
\Gamma_{\mathrm{red}}^{\mathrm{CW}} \leq\left(\frac{L_{\mathrm{CW}}}{z}\right),
\end{gathered}
$$

where the diffraction length $L_{\mathrm{Cw}}=A / \lambda_{\mathrm{Cw}}$ has been introduced.

Next, consider the far field efficiency of the same array driven with a set of LW functions. Here one has 


$$
\Gamma_{\text {nd }}^{L W} \leq\left(\frac{A}{2 \pi c z}\right)^{2} \frac{U_{\text {rad }}^{L W}}{\mathscr{E}_{\text {in }}^{L W}}
$$

Note that the CW array efficiency is conveniently given in (2.43) in terms of the CW diffraction length. However, for the LW driven array, the concept of a diffraction length is not readily applied since the LW consists of broadband frequency components. However, one may define an effective frequency that provides a quantitative measure of the frequency content of the LW beam. This effective frequency may then be used to determine the diffraction length, $L_{\mathrm{Lw}}=A f_{\text {eff }} / c=A / \lambda_{\text {eff }}$, where $\lambda_{\text {eff }}$ is the effective wavelength. Consider the effective frequency of the radiated field $\omega_{\text {rad, }}$ which is defined as

$$
\omega_{\text {nad }}^{2}=\frac{U_{\text {rad }}^{\mathrm{LW}}(\text { far field })}{\mathscr{E}_{\mathrm{in}}^{\mathrm{LW}}}=\frac{\sum_{n=1}^{N} A_{n} \int_{-\infty}^{\infty} d t\left|\partial_{t} \psi_{n}\left(\mathbf{r}_{n}, t\right)\right|^{2}}{\sum_{n=1}^{N} A_{n} \int_{-\infty}^{\infty} d t\left|\Psi_{n}\left(\mathbf{r}_{n}, t\right)\right|^{2}}=\frac{\sum_{n=1}^{N} A_{n} \int_{-\infty}^{\infty} d \omega \omega^{2}\left|\Psi_{n}\left(\mathbf{r}_{n}, \omega\right)\right|^{2}}{\sum_{n=1}^{N} A_{n} \int_{-\infty}^{\infty} d \omega\left|\Psi_{n}\left(\mathbf{r}_{n}, \omega\right)\right|^{2}} .
$$

In (2.45), the term far field refers to the situation where the approximation $R \approx z$ is valid. Because the measurement process alters the frequency content of the beam, an effective frequency will also have to be described for the measured field as well. This will be discussed later.

Now that the concept of effective frequencies has been defined for the LW radiated field, the efficiency of the $\mathrm{LW}$ radiated field may be given in terms of the diffraction length. 


$$
\Gamma_{\text {rad }}^{\text {LW }} \leq\left(\frac{L_{\text {red }}}{z}\right) \text {, }
$$

where the wavelength $\lambda_{\text {rad }}=2 \pi c / \omega_{\text {rad }}$ and the Rayleigh distance is defined as $L_{\mathrm{rad}}=A / \lambda_{\mathrm{rad}}$. Hence, the radiated field efficiency of the LW pulse can be measured in relation to that of the $\mathrm{CW}$ pulse by taking the ratio of two efficiencies

$$
\frac{\Gamma_{\mathrm{rad}}^{\mathrm{Lw}}}{\Gamma_{\mathrm{rad}}^{\mathrm{Cw}}} \approx\left(\frac{\omega_{\mathrm{rdd}}}{\omega_{\mathrm{cw}}}\right)^{2}
$$

The question is whether or not this ratio is greater than one. In order to determine this, the value of $\omega_{\mathrm{CW}}$ that makes a fair comparison of the $\mathrm{CW}$ and $\mathrm{LW}$ pulses should be quantified. For instance, the effective frequency radiated field very close to array, $\boldsymbol{\Omega}_{\text {rad }}$, may also be defined in the same way that $\omega_{\text {rad }}$ was,

$$
\Omega_{\text {rad }}^{2}=\frac{U_{\text {rad }}^{\text {LW }}(\text { near field })}{\mathscr{E}_{\text {in }}^{\text {LW }}}=\frac{\sum_{n=1}^{N} A_{n} \int_{-\infty}^{\infty} d t\left|\Phi_{n}\left(\mathbf{r}_{n}, t\right)\right|^{2}}{\sum_{n=1}^{N} A_{n} \int_{-\infty}^{\infty} d t\left|\int_{-\infty}^{t} d \tau \Phi_{n}\left(\mathbf{r}_{n}, \tau\right)\right|^{2}} .
$$

In (2.45), it was assumed that the observation point is located spatially far away from the array (i.e. $R \approx z$ ) and that the transducer driving functions may be given by $\psi_{n}$, where $\Phi_{n} \approx 2 \partial_{a} \psi_{n}$. In this case, the observation point is located spatially near the array (i.e. is located in the array's near field), where $\Phi$ must be given by (2.29). In this case, the driving functions are given by $\int_{\infty}^{\ell} d \tau \Phi\left(\mathbf{r}_{n}, \tau\right) / 2$. The value $\Omega_{\text {rad }}$ is an array weighted accumulation of the input LW signal spectra and is thus a measure of the input field 
spectra. Hence $\Omega_{\text {rad }}$ may be considered a fair substitute for $\omega_{\mathrm{CW}}$ in (2.47). Note that it can be shown that $\Omega_{\mathrm{rad}}<\omega_{\text {rad }}$. The standard engineering criterion of the $3 \mathrm{~dB}$ frequency, $\omega_{3 \mathrm{~dB}}$, may also be used in place of $\omega_{\mathrm{CW}}$, in which case $\omega_{3 \mathrm{~dB}}<\omega_{\text {rad }}$. Other substitutions for $\omega_{\mathrm{cw}}$ may be made, however, as a demonstration, Ziolkowski [1991] chose the value of $\Omega_{\mathrm{rad}}$ for comparison, in which case

$$
\frac{\Gamma_{\mathrm{rad}}^{\mathrm{LW}}}{\Gamma_{\mathrm{rad}}^{\mathrm{CW}}} \approx\left[\frac{\omega_{\mathrm{red}}}{\omega_{\mathrm{cW}}=\Omega_{\mathrm{rad}}}\right)^{2} \geq 1 .
$$

Hence, one can claim that the LW beam is more efficient in transmitting its energy than the corresponding CW one. In an optimal design, one would try and maximize the value of the ratio given in $(2.49)$.

\subsubsection{Measured Field Efficiency}

The above radiated field analysis can also be performed on the measured field. As was discussed earlier, the measured field will differ from the radiated field by two time derivatives and a constant (equations (2.32) and (2.33)). In the same sense as described above, a measured field effective frequency can be quantified

$$
\omega_{\text {meas }}^{4}=\frac{\sum_{n=1}^{N} A_{n} \int_{-\infty}^{\infty} d t\left|\partial_{t}^{3} \psi_{n}\left(\mathbf{r}_{n}, t\right)\right|^{2}}{\sum_{n=1}^{N} A_{n} \int_{-\infty}^{\infty} d t\left|\partial_{\imath} \psi_{n}\left(\mathbf{r}_{n}, t\right)\right|^{2}} .
$$

If the array were driven with the same $\mathrm{CW}$ signal, $\omega_{\text {meas }}$ would be simply equal to $\omega_{\mathrm{CW}}$. 
The ratio of the $\mathrm{LW}$ to $\mathrm{CW}$ measured efficiencies is

$$
\frac{\Gamma_{\text {meas }}^{\mathrm{LW}}}{\Gamma_{\text {meas }}^{\mathrm{CW}}} \approx\left(\frac{\omega_{\text {rad }}}{\omega_{\mathrm{cW}}}\right)^{2}\left(\frac{\omega_{\text {mena }}}{\omega_{\mathrm{cW}}}\right)^{4} .
$$

Hence the condition for the LW array to be more efficient than the CW array is given by

$$
\omega_{\text {meas }}^{2}>\frac{\omega_{\mathrm{CW}}^{3}}{\omega_{\text {rad }}} .
$$

If the $\mathrm{CW}$ frequency is conservatively set to $\omega_{\mathrm{rad}}$ then $(2.52)$ becomes

$$
\omega_{\text {meas }}>\omega_{\text {rad }} \text {. }
$$

Ziolkowski has shown that the above criterion is readily achieved with a variety of LW solutions, as well as other broadband driving signals. As will be discussed here, Ziolkowski's experiments [Ziolkowski \& Lewis, 1989; 1990] showed that more than an order of magnitude increase in the measured field energies can be achieved.

\subsubsection{Beam Divergence}

Another performance measure which can quantify the beam quality of a LW is the rate of beam divergence or spread. This can be quantified by examining the fluence profile of the beam. In this case, when the centre of the beam has propagated a distance, $z$, the radius at which the fluence profile has decreased to half its maximum is measured. This radius is called the half width at half maximum, $\rho^{\text {HWHM }}(z)$, and is a good measure of beam's transverse localization. The HWHM radius can be calculated from the appropriate array fluence expression. For instance, the HWHM radius of the far field measured energy profile occurs at 


$$
\rho_{\text {meas }}^{\text {HWHM }} \approx\left(\frac{\lambda_{\text {meas }}}{4 d_{\max }}\right) z,
$$

where $d_{\max }$ is the radius of the aperture. Hence the rate of beam divergence is given by $\theta_{\text {meas }}=\partial_{z} \rho_{\text {meas }}^{\text {HWHM }} \approx \lambda_{\text {meas }} / 4 d_{\max }$. Using the same analysis, the radiated field HWHM radius occurs at,

$$
\rho_{\text {rad }}^{\text {HWHM }} \approx\left(\frac{\lambda_{\text {rad }}}{4 d_{\max }}\right) z .
$$

Thus the beam generated by a LW array will have beam divergence given by $\theta_{\mathrm{rad}}^{\mathrm{LW}} \approx \lambda_{\mathrm{rad}} / 4 d_{\max }$, while the beam generated by a CW driven array is given by $\theta_{\text {rad }}^{\mathrm{CW}}=\theta_{\mathrm{mess}}^{\mathrm{CW}} \approx \lambda_{\mathrm{CW}} / 4 d_{\max }$. The ratio of the $\mathrm{LW}$ and $\mathrm{CW}$ beam divergence is given by

$$
\frac{\theta_{\text {meas }}^{\mathrm{LW}}}{\theta_{\text {meas }}^{\mathrm{CW}}} \approx \frac{\omega_{\text {meas }}^{\mathrm{CW}}}{\omega_{\text {mess }}^{\mathrm{LW}}} .
$$

As was discussed in the previous section, the ratio given (2.56) can be made much less than one by properly designing the correlation properties of the LW time signals. Hence, the beam generated by a LW array can be made more localized than a beam generated by a corresponding $\mathrm{CW}$ array. 


\subsection{Experimental Results}

\subsubsection{Simple Array}

Ziolkowski [1991] first considered the case of driving a circular aperture (radius $R_{a}$ ) with the real part of the MPS solution, $\psi_{\text {MPs }}$ previously given in (2.25). His experiments used the parameters $z_{0}=4.5 \times 10^{-4} \mathrm{~m}, T=1, \tau=1.0 \mathrm{~m}, B=300$ and $b=600$ $\mathrm{m}^{-1}$.

In order to write compactly the diffraction lengths of the various beams, the waist, $w$, of the beam is introduced. The waist is usually defined as the beam radius at half maximum of the beam's intensity profile, $\left|\psi_{\text {MPS }}\right|^{2}$. The waist should not be confused with $\rho^{\text {HWHM }}$, which evaluates the transverse localization of the radiated and measured fields using the fluence profile. The initial waist of the MPS is given by $w=\left(B z_{0} / b\right)^{1 / 2}=1.5 \mathrm{~cm}$. The maximum effective frequency, $f_{\max }$, of the MPS is also introduced and is defined as the $1 / e$ fall off point in the Fourier amplitude of the MPS. This frequency is derived by examining the temporal Fourier transform of the MPS at $z=0$,

$$
\Psi_{M P S}(\rho, z=0, \omega) \approx \frac{1}{z_{0} T} \frac{2 \pi}{\omega_{\max }} u\left(\omega-\omega_{\min }\right) e^{-\left(\omega-\omega \omega^{\prime} / \omega\right.}-J_{0}\left(2(\rho / c)\left[\omega_{\min }\left(\omega-\omega_{\min }\right)\right]^{1 / 2}\right),
$$

where $u$ is the unit step function, $\omega_{\min }=b c / B$, and $\omega_{\max }=c / z_{0}$ is the $1 / e$ fall off point. For the MPS parameters listed above, $f_{\max } \approx 0.53 \mathrm{MHz}$. Again, $f_{\max }$ should not be confused with the radiated

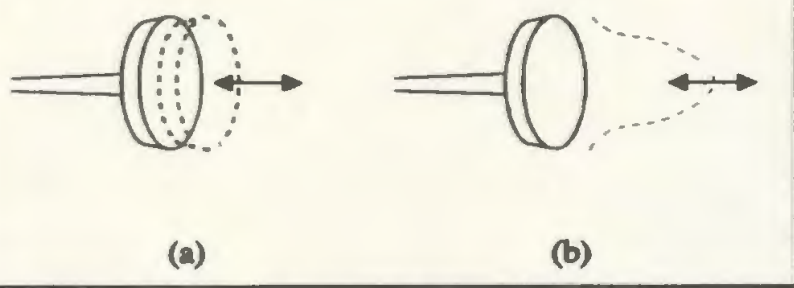

Figure 2.12: (a) Piston and (b) Gaussian beam. 
and measured effective frequencies, $f_{\text {rad }} \& f_{\text {meas }}$.

In these experiments, two different types of beams are compared with the MPS: a simple piston beam (aperture is uniformly illuminated with a $\mathrm{CW}$ signal) and a gaussian piston beam (aperture amplitude is tapered with a gaussian function in the transverse direction) as illustrated in figure 2.12. Using the expressions for the radiated field of an array from the previous section, the diffraction lengths of each of the particular beams can be derived,

$$
\begin{aligned}
& L_{\text {MPS }}=\frac{\pi w R_{a}}{\lambda_{\min }}, \\
& L_{\text {Giuss }}=\frac{\pi w^{2}}{\lambda_{\min }}, \\
& L_{\text {Pisao }}=\frac{\pi R_{a}^{2}}{\lambda_{\min }} .
\end{aligned}
$$

where $w \leq \boldsymbol{R}_{a}$. For the piston array, the entire aperture is illuminated with the CW signal having frequency, $f_{\max }$, while for the gaussian array, the aperture is driven with the transverse tapered CW signal, $f_{\max }$. From (2.58), it is seen that the MPS will have a longer diffraction length than the Gaussian piston if $w<\boldsymbol{R}_{a}$, while if this holds the regular piston will have the longest diffraction length.

As Ziolkowski [1991] points out, the diffraction length says nothing about the quality of the beam. While the $\mathrm{CW}$ piston has a longer diffraction length, its near field contains large regions of constructive and destructive interference, hence the beam quality is poor. In contrast, the Gaussian and MPS beam quality is pencil like, highly superior to that of the piston array. The theoretical waists of the piston and Gaussian 
beams are given by

$$
\begin{gathered}
w_{\text {Gauss }} \approx \frac{w z}{L_{\text {Gawse }}}=\frac{\lambda_{\min } z}{\pi w}, \\
w_{\text {Pison }} \approx \frac{\lambda_{\text {min }} z}{4 R_{a}} .
\end{gathered}
$$

Now consider the comparison of the MPS, Gaussian and piston beams using the radiated and measured diffraction lengths given in the previous section. The effective radiated and measured frequencies can be calculated, for the given MPS parameters, as

$$
\begin{gathered}
\omega_{\text {rad }}^{2} \approx \frac{3}{16} \omega_{\max }^{2}, \\
\omega_{\text {meas }}^{4} \approx\left[\frac{R_{a}^{2}}{2 w^{2}}\right]^{3} \omega_{\max }^{4} .
\end{gathered}
$$

Hence the radiated and measured field diffraction lengths are given by,

$$
\begin{gathered}
L_{\text {red }}^{\text {MPS }}=\frac{A}{\lambda_{\text {rad }}}=\frac{\pi R_{a}^{2}}{\lambda_{\text {rad }}} \approx \sqrt{3 / 16} L_{\text {Piseon }}=\sqrt{3 / 16}\left(\frac{R_{a}}{w}\right)^{2} L_{\text {Giuses }}, \\
L_{\text {mess }}^{\text {MPS }}=\left(\frac{\omega_{\text {meas }}}{\omega_{\text {rad }}}\right)^{2} L_{\text {red }} \approx \sqrt{16 / 3}\left[\frac{R_{a}^{2}}{2 w^{2}}\right]^{3 / 2} L_{\text {Piseon }}=\sqrt{2 / 3}\left[\frac{R_{a}}{w}\right]^{5} L_{\text {Oasusa }} .
\end{gathered}
$$

These theoretical results show that if $w<\boldsymbol{R}_{a}$, the MPS radiated field diffraction length can be made larger than that of the Gaussian array and the MPS measured field diffraction length can be made longer than the corresponding piston and Gaussian diffraction lengths. Similarly, the theoretical waists of the MPS beams are given by 


$$
\begin{gathered}
w_{\text {rad }}^{\text {MPS }} \approx \frac{\lambda_{\text {rad }}}{4 R_{a}}=\frac{4}{\sqrt{3}} w_{\text {Piston }}(z)=\frac{1.81 w}{R_{a}} w_{\text {Guuss }}(z) \\
w_{\text {meas }}^{\text {MPS }} \approx \frac{\lambda_{\text {meas }}}{4 R_{a}}=\left[\frac{2 w^{2}}{R_{a}^{2}}\right]^{3 / 4} w_{\text {Pirrou }}(z)=1.32\left[\frac{w}{R_{a}}\right] w_{\text {Gauss }}(z)
\end{gathered}
$$

Again, these results show that if $w<R_{a}$ the waist of the MPS radiated field can be made smaller than that of the Gaussian array and the waist of the MPS measured field can be made smaller the corresponding piston and Gaussian waists.

The experiments were carried out on a $6 \mathrm{~cm}$ square array consisting of $21 \times 21$ elements. The array was synthetic; a single transmitting element was used to synthesize an entire array. A circular array was obtained by zeroing the signals of the elements outside $R_{a}=3 \mathrm{~cm}$. Hence, the effective radiated and measured frequency values are calculated as $f_{\text {rad }}=0.23 \mathrm{MHz}, f_{\text {meas }}=0.89 \mathrm{MHz}$. The frequency of the piston and Gaussian beams is set to $f_{\max }=0.53 \mathrm{MHz}$. The theoretical measured Rayleigh distances for the MPS, Piston, and Gaussian beams compare ${ }^{1}$ as $L_{\text {meas }}^{\text {MPS }}=\left(\omega_{\text {meas }} / \omega_{\text {rad }}\right)^{2} L_{\text {rad }}=6.54 L_{\text {Pincon }}=$ $26.14 L_{\text {Gaus }}=6.53 \mathrm{~m}$. The measured Rayleigh distances for the piston and Gaussian beams are equal to their radiated field values. The experimentally measured Rayleigh distance of the MPS array was actually $150 \mathrm{~cm}$. Although there is a large discrepancy between the predicted and measured values of $L_{\text {meas }}^{\text {MPS }}$, the measured MPS Rayleigh distance exceeds the piston Rayleigh distance by a factor of $1.5, L_{\text {meas }}^{\text {MPS }}=1.5 L_{\text {Pistom }}$. The folded array results presented in the next section produced experimental diffraction

${ }^{1}$ Ziolkowski $\{1991\}$ in his original paper mistakenly writes $L_{\text {meas }}^{\text {MPS }}=\left(\omega_{\text {meas }} / \omega_{\mathrm{CW}}\right)^{2} L_{\text {rad }}$. This has been corrected here. 
lengths that more closely resemble the predicted diffraction lengths.

The theoretical waists of the three beams compare as $w_{\text {meas }}^{\text {MPS }}=0.59 w_{\text {meas }}^{\text {Piston }}$

$=0.23 w_{\text {meas }}^{\text {Caus }}$. Note that the beam divergence can be calculated from the waists by differentiating the waist with respect to $z$ (or in this case dividing by $z$ ). Hence the ratio of the MPS beam divergence to Gaussian beam divergence is approximately 4.29. Experimentally this value was determined to be 6.29. The discrepancy between theoretical and experimental values was attributed to experimental error.

\subsubsection{Folded Array}

One of the most important aspects of Ziolkowski's LW research involved determining how far beyond the classical Rayleigh distance, $L_{\text {Pisoo, }}$ the localized wave diffraction length would reach. As discussed previously, a larger array leads to a longer diffraction length for a given localized wave. Hence, Ziolkowski

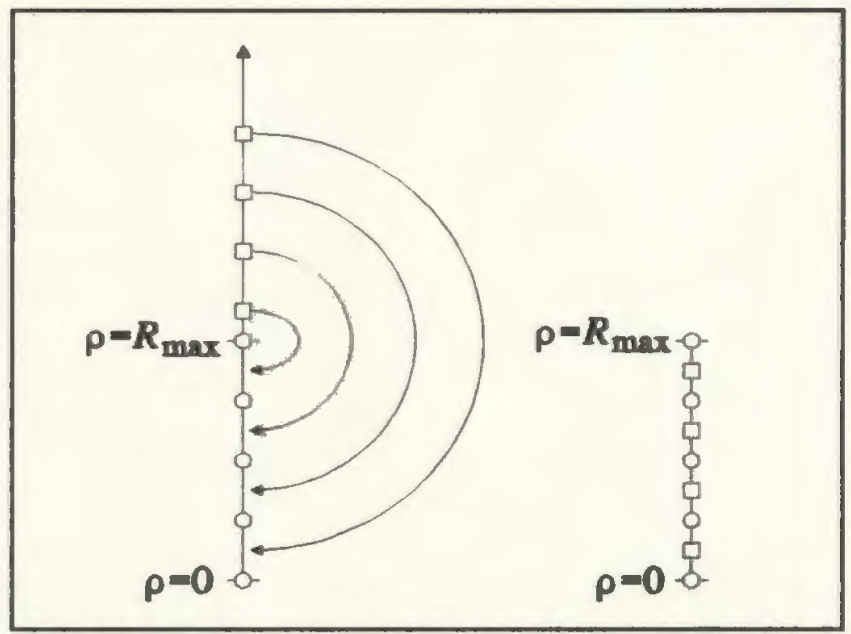

Figure 2.13: Elements are folded onto the aperture (left) to produce a folded array (right).

proposed squeezing a larger array into a

smaller one using a folded array scheme [1989] as depicted in figure 2.13. As shown in the figure, the aperture size is set to $R_{\max }$. Driving functions outside of the aperture can be folded onto the aperture using the conformal map $\rho \rightarrow R_{\max }^{2} / \rho$. Although the folded array produces better results than the simple array, the tradeoff is a much more 
complex driving source distribution. Hence it is much more difficult to derive the theoretical waists and Rayleigh distances in closed form as was done for the simple array. As Ziolkowski demonstrated, this tradeoff produced a ten-fold enhancement in the measured diffraction length.

As discussed in [Ziolkowski, 1991; Ziolkowski \& Lewis, 1990], the folded array was tested on a 25 element, $(5 \times 5)$ array. Array elements were spaced at $2.5 \mathrm{~mm}$ such that the array was $1.25 \mathrm{~cm}$ on a side with $A=1.5625 \mathrm{~cm}^{2}$. The array elements were driven with the folded scheme driving function

$$
\Phi(\rho, t)= \begin{cases}W(t) \psi_{\mathrm{MPS}}(\rho, z=0, t) & \rho=0 \\ W(t)\left[\psi_{\mathrm{MPS}}(\rho, z=0, t)+\left(R_{\max } / \rho\right)^{4} \psi_{\mathrm{MPS}}\left(R_{\max }^{2} / \rho, z=0, t-t_{d}\right)\right] & \rho \neq 0,\end{cases}
$$

where $t_{d}=\left[\left(z_{d}^{2}+\left(R_{\max }^{2} / \rho\right)^{2}\right)^{1 / 2}-\left(z_{d}^{2}+\rho^{2}\right)^{1 / 2}\right] / c$ is the position dependent delay time, $z_{d}$ is the constant delay distance $3.0 \mathrm{~cm}$, and $W(\mathrm{t})$ is a window function that removes the Mach cones characteristic to the MPS pulse (see figures 2.4-2.7).

The effective frequency values and diffraction lengths were calculated numerically. The radiated field effective frequency is given by, $f_{\text {rad }}=0.33 \mathrm{MHz}$. For comparison, the piston $\mathrm{CW}$ frequency is set to $f_{\mathrm{cw}}=1.52 f_{\text {rad }}=0.5 \mathrm{MHz}$ (Ziolkowski chooses this more conservative choice of $f_{\mathrm{cW}}$ over setting $f_{\mathrm{cw}}=f_{\mathrm{rad}}$ to illustrate the superior beam characteristics of the MPS). The LW radiated field Rayleigh distance can be calculated as $L_{\text {rad }}^{\text {MPS }}=0.66 L_{\text {Piston }}=3.44 \mathrm{~cm}$. The effective frequency of the measured field is given as $f_{\text {meas }}=1.435 \mathrm{MHz}$. From this, the measured field diffraction length is given by $L_{\text {areas }}^{\text {MPS }}=\left(f_{\text {meas }} / f_{\text {rad }}\right)^{2} L_{\text {rad }}^{\text {MPS }}=12.48 L_{\text {Pistoa }}=65.05 \mathrm{~cm}$. The beam divergence rate is 
$\theta_{\text {meas }}^{\mathrm{LW}}=2.02 \times 10^{-2}$. When compared to the piston beam, the measured efficiency ratio, $\Gamma_{\text {meas }}^{\mathrm{LW}} / \Gamma_{\text {meas }}^{\mathrm{CW}}$, of the MPS beam indicates an enhancement factor of 12.11 , and the beam divergence rate, $\theta_{\text {meas }}^{\mathrm{LW}} / \theta_{\text {meas }}^{\mathrm{CW}}$, indicates a decrease factor of 5.11 .

Both numerical simulations [Ziolkowski, 1991] and experiments [Ziolkowski \& Lewis, 1990] were conducted to confirm the predicted results given above. In the numerical experiments, $L_{\text {meas }}^{\mathrm{MPS}}=\left(f_{\text {mean }} / f_{\text {rad }}\right)^{2} L_{\text {rad }}^{\mathrm{MPS}}$ lay in the 50-60 cm region, $\Gamma_{\text {meas }}^{\mathrm{LW}} / \Gamma_{\text {meas }}^{\mathrm{CW}}$ was calculated to be 6.25 , while $\theta_{\text {mess }}^{\mathrm{LW}} / \theta_{\text {meas }}^{\mathrm{CW}}$ indicates a decrease factor of 3.66 . For the acoustic tank experiments, $L_{\text {Piston }}=3.0 \mathrm{~cm}, L_{\text {meas }}^{\text {MPS }}=\left(f_{\text {meas }} / f_{\text {mad }}\right)^{2} L_{\text {rad }}^{\text {MPS }}$ lay in the $50-60 \mathrm{~cm}$ region $\approx 25 L_{\text {Piston }}$, while $\theta_{\text {meas }}^{\mathrm{LW}} / \theta_{\text {meas }}^{\mathrm{CW}}$ indicates a decrease factor of 2.91 . The numerical and experimental data compare extremely well, while the experimental and predicted values compare reasonably.

Ziolkowski [1991] points out that the experimental results from both the simple and folded arrays show that LW arrays can be designed to effectively outperform CW arrays in the context of multi-time-derivative transmit/receive systems. In his experimentation, Ziolkowski used the MPS pulse, which is by no means the optimal LW pulse. The MPS pulse was only chosen because it has interesting propagation characteristics, and its closed form expression allows it to be easily analyzed and simulated. Further optimization work on localized waves is essential in determining the waves with optimal propagation characteristics. 


\section{Predicted Backscatter Spectrum}

\subsection{Backscatter Spectrum Calculation}

In what follows, the $e^{- \text {iks }}$ time dependence will be suppressed on field quantities, and, for reasons that will become evident, it will be assumed that $\omega<0$. As such, if $\mathbf{k}$ denotes a vector whose spherical polar components are $(k, \vartheta, \varphi)$, then the plane wave

$$
e^{i \mathbf{k} \cdot \mathbf{r}-i n s}
$$

is not travelling in the direction of $\mathbf{k}$, as $t$ increases. Writing

$$
e^{i \mathbf{k} \cdot \mathbf{r}-i \omega t}=\left[e^{-i \mathbf{k} \cdot \mathbf{r}-i|\omega| t}\right]^{*}
$$

it is evident that the plane wave $e^{-\mathbf{k} \cdot \cdot \cdot-i|\omega|^{\imath}}$ is travelling in the direction of $-\mathbf{k}$ as $t$ increases, where the spherical polar components of $-k$ are $(k, \pi-\vartheta, \varphi+\pi)$.

Morse and Ingard [1968] give an expression for the scattered field at a point $(r, \theta, \phi)$ due to a plane wave of unit amplitude and wave number $k$, incident on a nonrigid sphere of radius $a$ centred on the origin, in the direction of the positive $z$-axis. The medium outside the sphere is assumed to be homogeneous, isotropic, and have a velocity of wave propagation $c$. Symmetry considerations

(a)

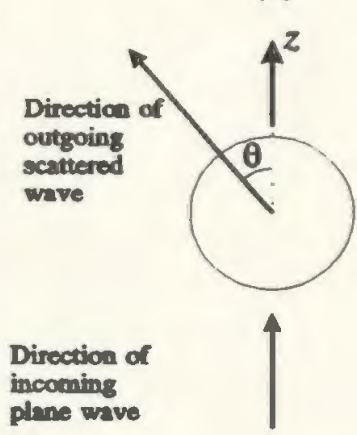

Figure 3.1: $\quad$ Symmetry relationship for scattering expression in (3.3). imply that this expression will also give the scattered field at a point whose Cartesian coordinates are $(0,0,-r)$, due to a plane wave travelling towards the centre of the sphere 
from a direction whose spherical polar angles are $\vartheta \& \phi$ (figure 3.1). According to (3.2) above, this result may be modified to obtain the field at $(0,0,-r)$ due to the plane wave

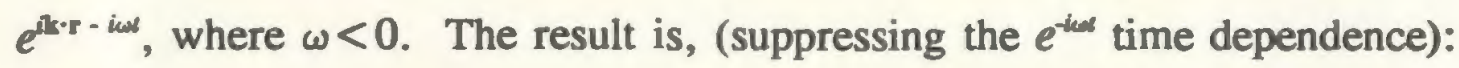

$$
-\left[\sum_{n=0}^{\infty}(i)^{n}(2 n+1) \frac{j_{n}(k a)+i \alpha_{n} j_{n}(k a)}{h_{n}^{(1) \prime}(k a)+i \alpha_{n} h_{n}^{(1)}(k a)} h_{n}^{(1)}(k r) P_{n}(\cos \vartheta)\right]
$$

In (3.3) the term $\alpha_{n}$ is given by

$$
\alpha_{n}=\frac{i \varrho c}{\varrho_{e} c_{e}}\left[\frac{j_{n}^{\prime}\left(k_{e} a\right)}{j_{n}\left(k_{e} a\right)}\right]
$$

where $\varrho$ is the density of the infinite medium surrounding the sphere, $\varrho_{e}$ is the density of the sphere material, $c_{e}$ is the speed of wave propagation in the sphere (so that $c_{e}=\sqrt{\varrho_{e} \kappa_{e}}$, where $\kappa_{e}$ is the compressibility of the sphere material), $k=|\omega| / c$, $k_{e}=|\omega| / c_{e}$, a prime superscript on a function denotes the derivative of that function, $j_{n}$ is the usual spherical Bessel function, $h_{n}^{(1)}$ is the spherical Hankel function of the first kind, and $\boldsymbol{P}_{n}$ is a Legendre polynomial. The expression in (3.3) may be compactly rewritten as

$$
\sum_{n=0}^{\infty} \gamma_{n} h_{n}^{(2)}(k r) P_{n}(\cos \vartheta)
$$

where

$$
\gamma_{n}=-(2 n-1)(-i)^{n}\left(\frac{j_{n}^{\prime}(k a)+i \alpha_{n} j_{n}(k a)}{h_{n}^{(2) \prime}(k a)+i \alpha_{n} h_{n}^{(2)}(k a)}\right)
$$

and where $h_{n}^{(2)}$ is the spherical Hankel function of the second kind. 
In principle then, one may obtain the scattered field at the point $(0,0,-r)$ on the negative $z$-axis, due to an arbitrary incident waveform, if it is composed of a weighted superposition of plane waves like (3.1). One merely takes the weighted superposition of (3.5) over each plane wave (i.e. Fourier) component of the incident waveform (the weighting being the spacetime Fourier transform of the incident waveform).

It will be assumed that the incident plane wave, $\psi(r, t)$, is a solution of the HWE for an infinite space filled with material such as that surrounding the sphere. The example used here will be the FWM solution discussed previously in chapter 2. The Fourier transform of the FWM is repeated here in (3.7) along with its support line projected onto the $k_{z}, \omega / c$

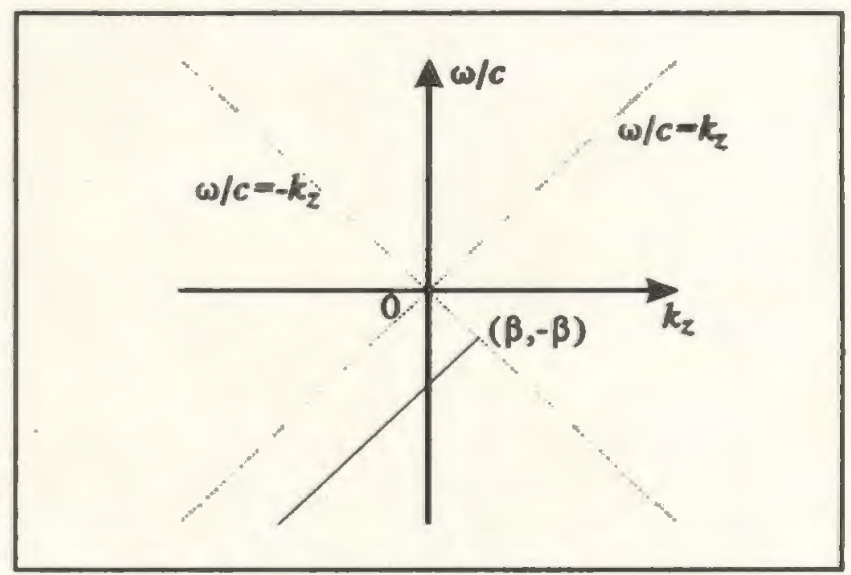

Figure 3.2: Projection of the support line of $\Psi_{\text {FWM }}(k, \omega, \beta)$, as given in (3.7) onto the $k_{z}$, $\omega / c$ plane (projection shown as solid line segment).

plane (figure 3.2)

$$
\Psi_{\mathrm{FWM}}(\mathrm{k}, \omega, \beta)=\frac{\pi^{2}}{i \beta} e^{-k^{2} z / 4 \beta} \delta\left[k_{z}-\left(\beta-\kappa^{2} / 4 \beta\right)\right] \delta\left[\omega+c\left(\beta+\kappa^{2} / 4 \beta\right)\right] .
$$

From figure 3.2 it is seen that the temporal Fourier transform of the FWM, $\mathscr{T}_{\{}\left\{\psi_{\mathrm{FWM}}\right\}(\mathbf{r}, \omega, \beta)$ will be an analytic signal, in that it contains only negative frequency components. The signal that is launched in a practical experiment is an approximation to $\operatorname{Re}\left\{\psi_{\mathrm{FWM}}\right\}(\mathbf{r}, t, \beta)$, which has the two sided temporal Fourier transform $1 / 2\left[\mathscr{F}_{i}\left\{\psi_{\mathrm{FWM}}\right\}(\mathbf{r}, \omega, \beta)+\left(\mathscr{F}_{\mathfrak{l}}\left\{\psi_{\mathrm{FWM}}\right\}(\mathbf{r}, \omega, \beta)\right)\right]$. 
As discussed above then, the scattered field at the point whose Cartesian coordinates are $(0,0,-r)$, due to an incident FWM of the form $(2.9)$, will be given by a weighted superposition of the expression (3.5), for a single plane wave, over the plane wave [Fourier] decomposition (2.8) of the FWM. That is, the scattered field is given by

$$
\begin{gathered}
\Lambda_{\beta}(r, \omega)=\frac{\pi^{2}}{i \beta} \frac{1}{(2 \pi)^{3}} \int_{\mathbf{R}^{3}} d \mathbf{k} e^{-k^{2} z^{\prime} / 4 \beta} \delta\left[k_{z}-\left(\beta-\kappa^{2} / 4 \beta\right)\right] \delta\left[\omega+c\left(\beta+\kappa^{2} / 4 \beta\right)\right] \\
\times \gamma_{n} h_{n}^{(2)}(k r) P_{n}(\cos \vartheta)
\end{gathered}
$$

where $\int_{\mathbf{R}^{\prime}} d \mathbf{k}$ denotes the integral over the three dimensional spatial Fourier transform domain.

The following identity, valid in the sense of generalized functions, is readily verified:

$$
\delta\left[\omega+c \beta+c \kappa^{2} / 4 \beta\right] \equiv \frac{\sqrt{\beta}}{c \sqrt{|\beta+\omega / c|}} u(-\omega / c-\beta) \delta[\kappa-\sqrt{4 \beta|\beta+\omega / c|}],
$$

where $u$, the unit step function, is needed to ensure $\omega+c \beta<0$. The volume integral $\int_{\mathbf{R}^{\prime}}$ in (3.8) may be broken down into separate integrations over cylindrical polar coordinate variables as $\int_{0}^{2 \pi} d \varphi \int_{\mathbb{R}} d k_{2} \int_{0}^{\infty} d \kappa \kappa$. With the aid of the identity (3.9) the $\kappa \& \varphi$ integrations may be performed to give 


$$
\begin{aligned}
\Lambda_{\beta}(r, \omega) & =\frac{u(-\beta-\omega / c)}{2 i c} e^{z_{(}(\beta+\omega / c)} \\
& \times\left.\int_{\mathbb{R}} d k_{z} \delta\left[k_{z}-(2 \beta+\omega / c)\right] \sum_{n=0}^{\infty} \gamma_{n} h_{n}^{(2)}(k r) P_{n}(\cos \vartheta)\right|_{\kappa=[\alpha \beta|\beta+\omega / c|]^{2 n}} .
\end{aligned}
$$

Care must be exercised with the remaining $k_{z}$ integral. With $\kappa=\sqrt{4 \beta|\beta+\omega / c|}$, it is seen that the remaining delta function will set $k_{z}$ so that $k$ becomes

$$
k=\sqrt{\kappa^{2}+k_{z}^{2}}=\left\{4 \beta|\beta+\omega / c|+(2 \beta+\omega / c)^{2}\right\}^{1 / 2}=|\omega| / c
$$

where the last equality followed from the step function $u(-\omega / c-\beta)$ in (3.10). Similarly, it can be shown that $\cos \vartheta=k_{z} / k=\{2 \beta \mathrm{c} /|\omega|\}-1$. Thus, (3.10) becomes

$$
\Lambda_{\beta}(r, \omega)=\left.\frac{u(-\beta-\omega / c)}{2 i c} e^{z_{0}(\beta+\omega / c)} \sum_{n=0}^{\infty} \gamma_{n}\right|_{k=|\omega| / c} h_{n}^{(2)}(|\omega| r / c) P_{n}\left[\frac{2 \beta c}{|\omega|}-1\right] .
$$

As was discussed chapter in 2, a LW finite energy solution to the HWE may be obtained by taking a weighted superposition over the arbitrary parameter $\beta$,

$$
\int_{0}^{\infty} d \beta F(\beta) \psi_{\mathrm{FWM}}(\mathbf{r}, t, \beta)
$$

with appropriate conditions on the integrability of $F$. In particular, the weighting

$$
F(\beta)= \begin{cases}4 \pi i \beta e^{-(B \beta-b)}, & \text { if } \beta>b / B \\ 0 & \text { if } 0 \leq \beta<b / B .\end{cases}
$$

is chosen to obtain the MPS superposition (the parameters $\tau$ and $T$ have been set to unity in equation (2.25)) [Ziolkowski, 1985].

With the waveform (2.25) incident on the sphere, the received signal at the point 
$(0,0,-r)$ on the negative $z$-axis is given by

$$
\begin{aligned}
\Lambda_{\mathrm{MPS}}(r, \omega)= & \int_{0}^{\infty} d \beta F(\beta) \Lambda_{\beta}(r, \omega) \\
= & \left.\frac{2 \pi B u(-\omega / c-b / B) e^{z^{2 \omega / c} / b}}{c} \sum_{n=0}^{\infty} \gamma_{n}\right|_{k=|\omega| / c} h_{n}^{(2)}(|\omega| r / c) \\
& \times \int_{b / B}^{|\omega| / c} d \beta e^{-\beta\left(B-z_{0}\right)} P_{n}((2 \beta c) /|\omega|-1)
\end{aligned}
$$

If the change of variable $\zeta=\{2 \beta c /|\omega|\}-1$ is made in (3.15), the result can be integrated by parts $n$ times $\left(P_{n}^{(n+1)} \equiv 0\right)$ to get

$$
\begin{aligned}
\Lambda_{\mathrm{MPS}}(r, \omega)= & \left.\frac{\pi B|\omega| u(-\omega / c-b / B)}{c^{2}} \sum_{n=0}^{\infty} \gamma_{n}\right|_{k=|\omega| / c} h_{n}^{(2)}(|\omega| r / c) \\
& \times\left(e^{-z_{(j /|\omega| / c-b / B)}}\left[\frac{P_{n}\left(\zeta_{0}\right)}{\sigma}+\frac{P_{n}^{\prime}\left(\zeta_{0}\right)}{\sigma^{2}}+\cdots+\frac{P_{n}^{(n)}\left(\zeta_{0}\right)}{\sigma^{n+1}}\right]\right. \\
& \left.-e^{-|\omega| B / c+b}\left[\frac{P_{n}(1)}{\sigma}+\frac{P_{n}^{\prime}(1)}{\sigma^{2}}+\cdots+\frac{P_{n}^{(n)}(1)}{\sigma^{n+1}}\right]\right)
\end{aligned}
$$

where

$$
\sigma=\frac{|\omega|}{2 c}\left(B-z_{0}\right) \quad \zeta_{0}=\frac{2 b c}{B|\omega|}-1
$$

\subsection{Backscatter Spectrum Analysis}

Closer examination of (3.16) reveals that the predicted backscattered spectrum is a complicated function of the distance from the sphere, $r$, the sphere radius, $a$, and the sphere's material parameters, $\varrho_{e}$ and $c_{e}=\sqrt{\varrho_{e} \kappa_{e}}$. For remote sensing purposes, the 
ability to extract material parameters from

the sphere's backscattered spectrum would be valuable. Here, emphasis will be placed on the extraction of the sphere radius. In figure 3.3 , the predicted backscattered spectrum is illustrated in a two dimensional plot for sphere radius. In this example, the material parameters $c_{e}=3.0 \times 10^{3} \mathrm{~m} / \mathrm{s}$ and $\varrho_{e}=7.8 \times 10^{3} \mathrm{~kg} / \mathrm{m}^{3}$, are used, similar to those of mild steel. The MPS parameters are set to $z_{0}=0.004$ $\mathrm{m}, \boldsymbol{B}=300$ and $b=750 \mathrm{~m}^{-1}$ for this and all subsequent examples and experiments. For particular values of $a$, as illustrated in figure figure 3.4 , the spectrum appears as a series of frequency peaks and dips

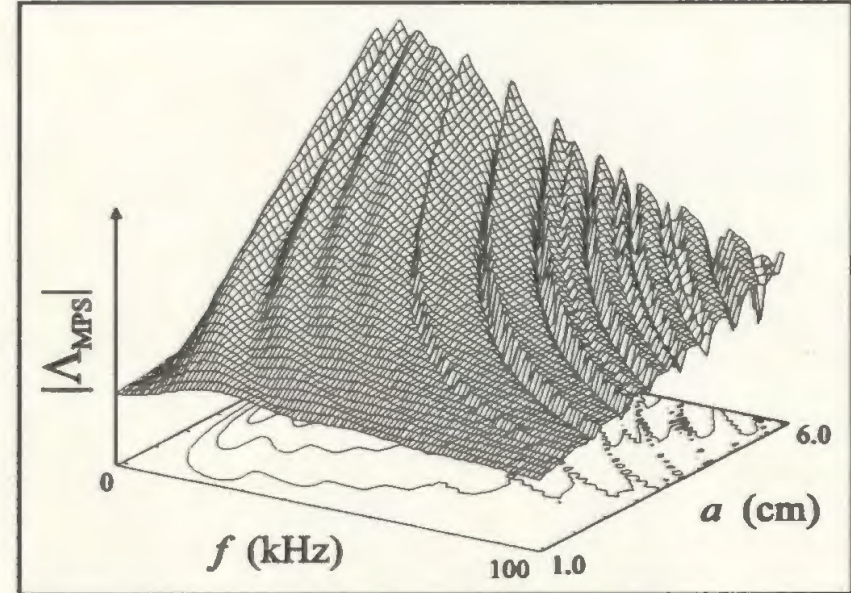

Figure 3.3: $\quad$ Surface plot of predicted backscattered spectra for varying sphere radius.

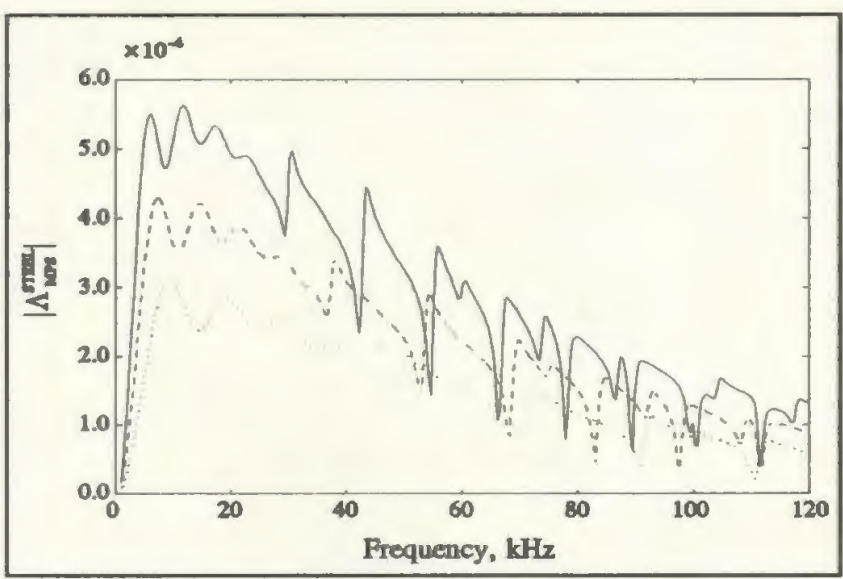

Figure 3.4: $\left|\Lambda_{\mathrm{MPS}}(r, \omega)\right|$ for $r=150 \mathrm{~mm}$, $c_{e}=3000 \mathrm{~m} / \mathrm{s}$, and $\varrho_{e}=7.8 \times 10^{3} \mathrm{~kg} / \mathrm{m}^{3}$ : $a=$ [50 (solid), 40 (dashed), and 30 (dotted)] $\mathrm{mm}$.

beyond approximately $30 \mathrm{kHz}$. The spectral spacing of these dips changes with sphere radius; as the size of the sphere increases, the spacing between adjacent dips decreases. Hence, the sphere radius may be extracted by examining the frequency of these spectral peaks and dips.

However, the variables $c_{e}$ and $\varrho_{e}$ also appear in (3.16); hence their effect on the spectral dip frequencies should be examined as well. In figure 3.5 , the backscattered 
spectrum is illustrated for varying $\varrho_{e^{-}}$. In this example the parameters $a=0.3 \mathrm{~cm}$ and $c_{e}=3.0 \times 10^{3} \mathrm{~m} / \mathrm{s}$ are used with $\varrho_{e}$ in the range $[3.0,12.0] \times 10^{3} \mathrm{~kg} / \mathrm{m}^{3}$. As the contour plot in this figure shows, a large change in $\varrho_{e}$ does not greatly affect the spacing of the spectral dips.

In figure 3.6 , the backscattered spectrum is illustrated for varying $c_{\ell}$. In this example, the sphere parameters $a=0.3 \mathrm{~cm}$ and $\varrho_{e}=7.8 \times 10^{3} \mathrm{~kg} / \mathrm{m}^{3}$ are used with of $c_{e}$ varying in the range [2.0, $4.0] \times 10^{3} \mathrm{~m} / \mathrm{s}$. This figure shows that as $c_{e}$ increases, the spacing of the spectral dips decreases. Hence, it seems that the backscattered spectrum changes with the

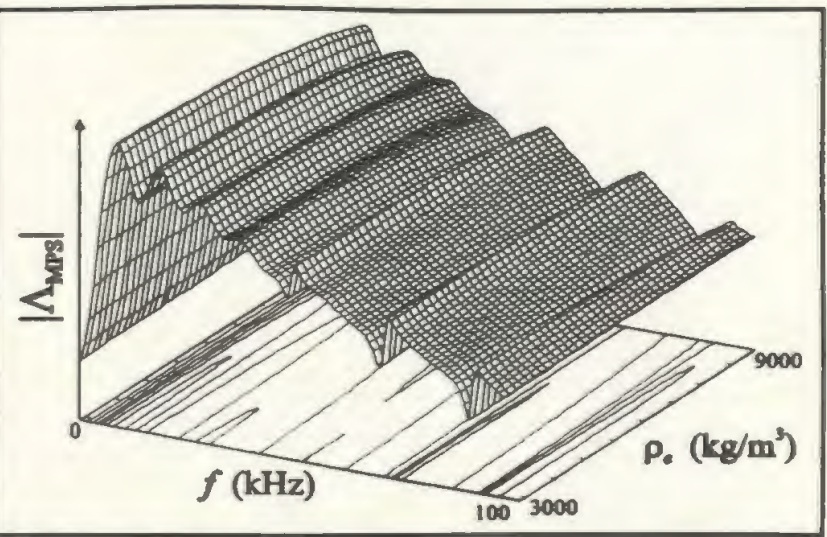

Figure 3.5: $\quad$ Surface plot of predicted backscattered spectra for varying sphere density.

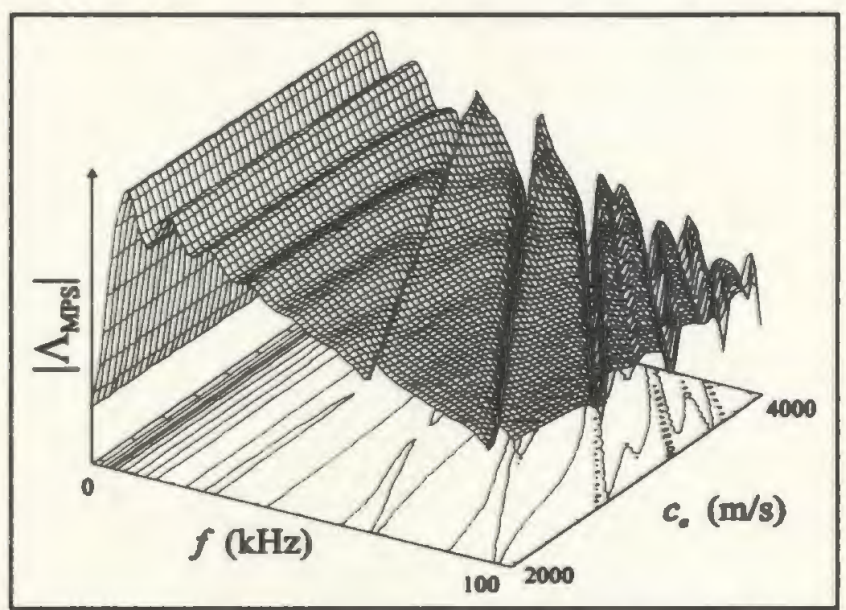

Figure 3.6: $\quad$ Surface plot of predicted backscattered spectra for varying $c_{e}$. sphere's speed of sound, yet doesn't change greatly with varying sphere density. This is illustrated more fully by fixing the frequency and changing both the sphere's density and speed of sound. The resulting surface plot is illustrated in figure 3.7. As this figure shows, the predicted backscattered spectrum does not change greatly as the density is changed yet changes abruptly as the speed of sound is changed.

It is notable that many metals and hard materials have similar $c_{e}$ values [Gray, 
1972] (table I). Hence it should be possible to extract the sphere size from the backscattered spectrum if the sphere is assumed to be a material with $c_{e}$ near $3100 \mathrm{~m} / \mathrm{s}$. The accuracy of the extraction will of course degrade as the actual value of $c_{e}$ varies away from the assumed value.

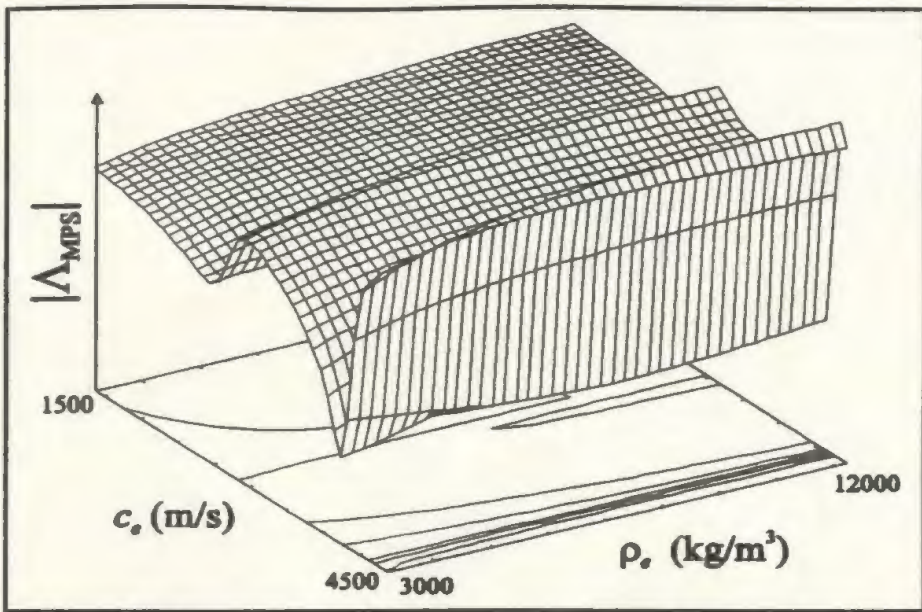

Figure 3.7: $\quad$ Surface plot of predicted backscattered spectra at $50 \mathrm{kHz}$ for varying $e_{e}$ and $c_{e}$.

\begin{tabular}{|c|r|}
\hline Material & $c_{e}(\mathrm{~m} / \mathrm{s})$ \\
\hline Stainless Steel & 3100 \\
\hline Aluminum (rolled) & 3040 \\
\hline Titanium & 3125 \\
\hline Nickel & 3000 \\
\hline Magnesium & 3050 \\
\hline Cast Iron & 2809 \\
\hline Pyrex Glass & 3280 \\
\hline
\end{tabular}

Table I: Speed of Sound in Various Rigid Materials 


\section{Experimental Methodology}

In order to obtain spherical backscatter signals for analysis, acoustic experiments were conducted in a large $(3 \times 4 \times 5 \mathrm{~m})$ tank. Three steel spheres with radii measuring 20,40 and $60 \mathrm{~mm}$, and a single aluminum sphere with a $40 \mathrm{~mm}$ radius, were used. The MPS pulses were launched using the double hydrophone system illustrated in figure 4.1 (next page).

The experimental setup is controlled using a single HP 9000 series PC and an IEEE-488 (GPIB) bus. This system allows for completely automated data aquisition. Digitized transmit signals are generated by the computer and sent via the GPIB to an arbitrary waveform generator and amplifier. Received signals are digitized and relayed back to the PC, again via the GPIB. A Kruger stepper motor/controller allows for movement of the transmitter through three axes.

\subsection{Transmitter System}

The transmitter (TX) system consists of a digital to analog converter (DAC), a power amplifier, and an ultrasonic transmitter. The DAC, a WaveTek model 275, has the ability to store a 2048 sample signal in memory and convert it using a definable sampling period (in the $267 \mathrm{~ns}-267 \mathrm{~ms}$ range). The WaveTek has a burst mode which allows a previously stored signal to be converted with a single GPIB command. The output analog signal consists of flat voltage steps which are smoothed by the filter in the power amplifier. The sampling frequency used throughout the experiments is $1 \mathrm{MHz}$.

The power amplifier, a $\mathbf{B} \& \mathrm{~K}$ model 2713 , has a linear frequency response and 


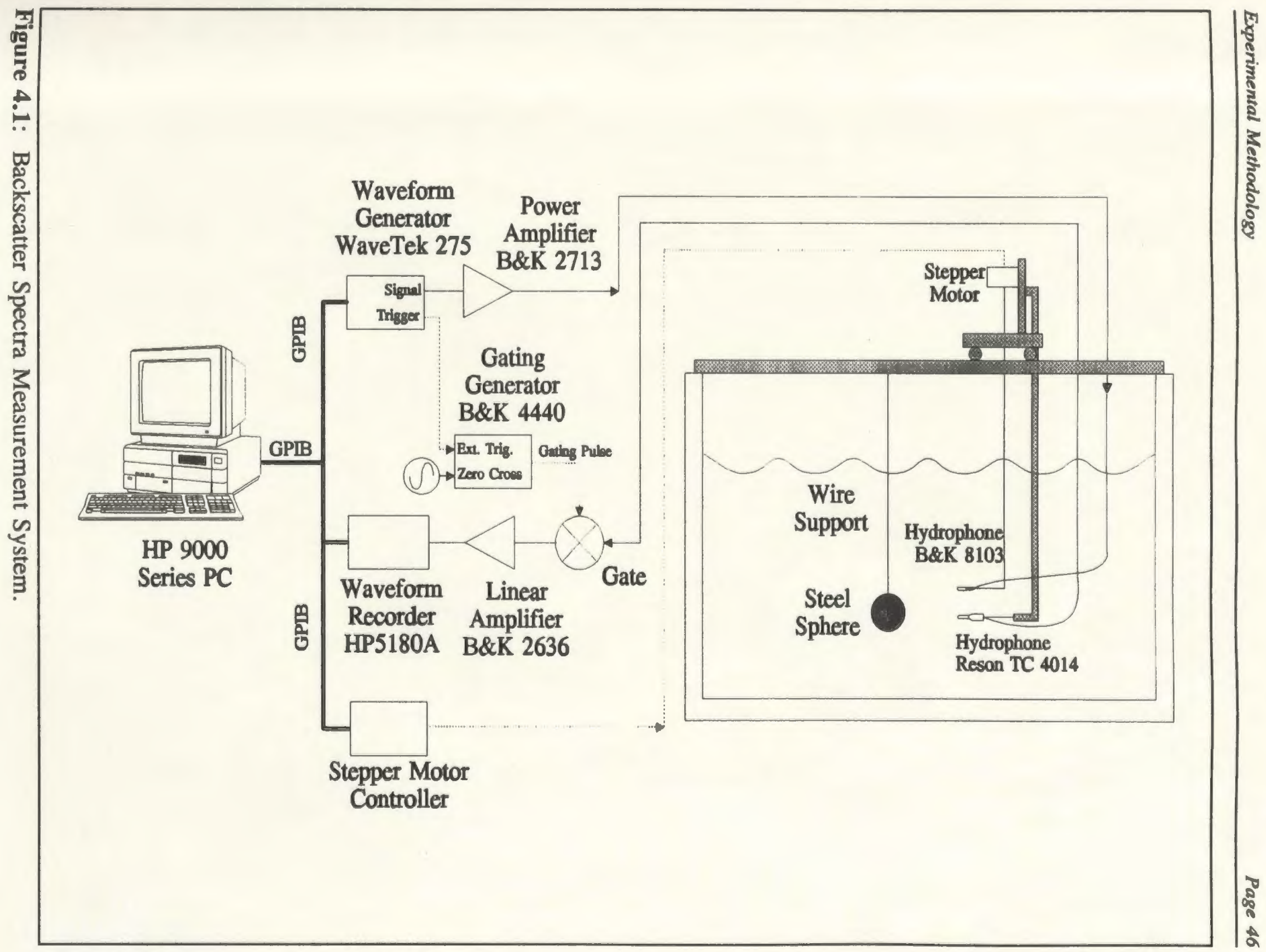


a bandwidth of $200 \mathrm{kHz}$. The $200 \mathrm{kHz}$ cutoff in the amplifier provides anti-aliasing filtering for the WaveTek analog signal.

The MPS pulses are launched synthetically, using superposition, by a Bruel and Kyjer hydrophone (B\&K 8103). A stepper motor is used to move the hydrophone to the required positions along the synthetic transmit aperture. The spheres are placed directly in front of the transmit aperture in line with the

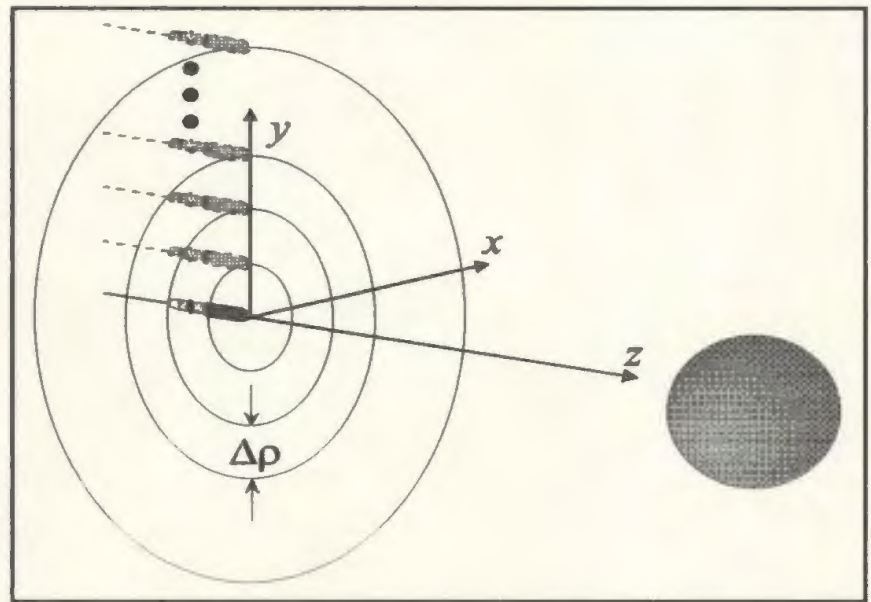

Figure 4.2: Sphere placed along the $z$-axis of the aperture.

array's $z$-axis (figure 4.2). The receiver hydrophone is placed near the centre of the transmit array, offset by about $1 \mathrm{~cm}$ from the $z$-axis. At this position, the receiver is assumed to be close enough to the centre of the array such that the signal it receives is approximately equal to the signal at the centre of the array (the backscattered signal).

In order to obtain a variety of spectra, the array was placed at distances of 150 , 250 , and $350 \mathrm{~mm}$ from the centre of each sphere. Because of the placement of the receiver, these distances represent the variable $r$ given previously in (3.16). At the distance $r=150 \mathrm{~mm}$, the spheres are located in the near field of the transducer array. In this case, the near field of the array simply refers to where the approximation $z / R \approx 1$, discussed previously in chapter 2 , is invalid (or where $z<$ size of aperture).

The frequency response of the transmit hydrophone, as measured $1 \mathrm{~m}$ away from the hydrophone, has a slope of approximately $40 \mathrm{~dB} /$ decade (figure 4.3 ) $10-110 \mathrm{kHz}$. In 
the time domain, this translates into a double time derivative as the signal passes through the hydrophone into the far field of the hydrophone. This phenomenon is similar to the disk transducer behaviour described by previously in chapter 2 in which the conversion from electrical energy to far field pressure is described

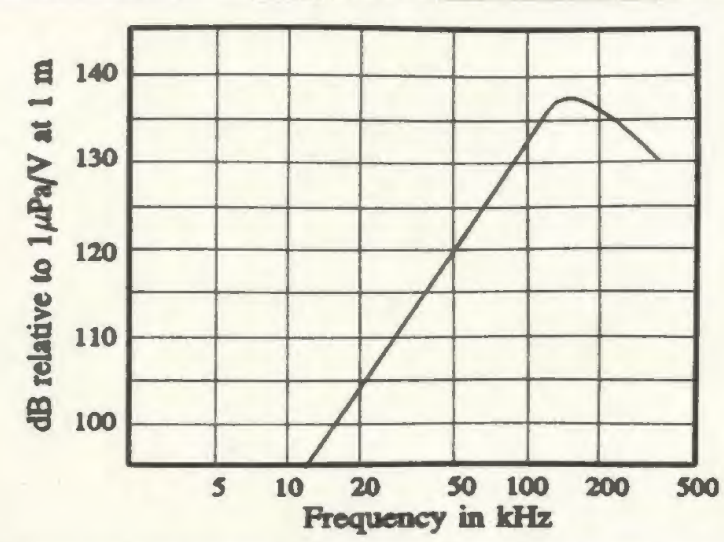

Figure 4.3: Frequency response of B\&K 8103 measured in water $1 \mathrm{~m}$ away from the hydrophone [Brüel and Kjær, 1986].

by two time derivatives. The transmitter is essentially omni-directional over the $x-y, y-z$ and $x-z$ planes below $110 \mathrm{kHz}$.

\subsection{Receiver System}

The receiver (RX) system consists of an ultrasonic receiver, a gating system, and a waveform recorder. The receiver hydrophone, a Reson TC4104, senses both the direct and reflected signals from the transmitting hydrophone and the sphere, respectively. The frequency response of the receiver is approximately flat below $400 \mathrm{kHz}$ (figure 4.4). The receiver is omni-directional over $360^{\circ}$ in the $x-y$ plane and $310^{\circ}$ in the $x-z, y-z$ plane below $200 \mathrm{kHz}$.

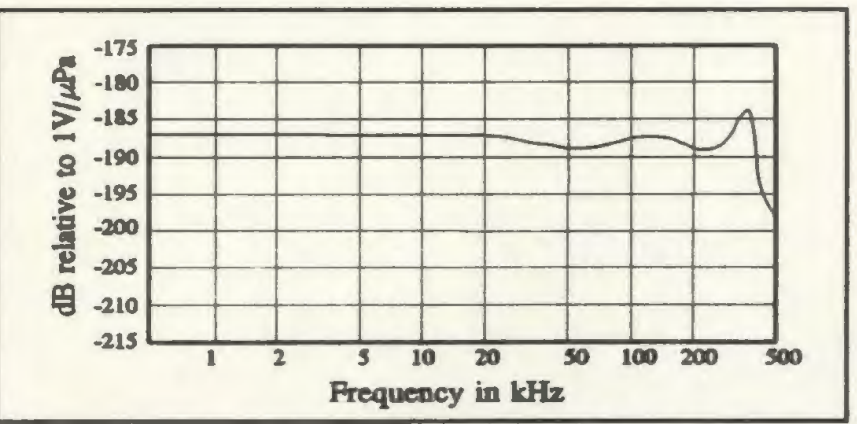

Figure 4.4: Frequency response of Reson TC-4104 [Reson System APS, 1988]

A gating system is used to remove a significant portion of the direct signal from 
the receiver signal so as to avoid saturation of the waveform recorder's input amplifiers. The gate consists of a gating generator (B\&K 4440) and an analog gate. The gating generator receives a trigger pulse from the DAC when the DAC starts sending its analog signal. Consequently, the generator produces an "on" voltage pulse that is fed into the analog gate. The delay and duration of the voltage pulse is controlled from the front panel of the gating generator. The waveform recorder converts the gated analog signal to digital form and relays it back to the PC via the GPIB.

The waveform recorder is used to convert the gated analog signals to digital form. The recorder was set up to receive 512 samples at a sampling rate of $1 \mathrm{MHz}$. Waveform recording is initiated by a triggering pulse sent by the DAC when it starts to transmit. The delay between when the recorder receives the trigger pulse and when is starts recording a waveform is also user definable.

\subsection{System Transfer Function}

As described in the previous two sections, the TX system imparts a double time derivative on an input signal in the range $0-110 \mathrm{kHz}$. On the other hand, the $\mathrm{RX}$ system essentially has a flat frequency response below $400 \mathrm{kHz}$. Hence, the TX/RX system in water will impart two time derivatives on any input signal in the $0-110 \mathrm{kHz}$ band. Analysis of system TX/RX transfer function was performed by inputting $(\sin 2 B t) / 2 B t$ pulses of bandwidth $B$ into the transmitter. A plot of the received signal from a $150 \mathrm{kHz}$ sinc pulse is shown in figure 4.5 .

Below approximately $110 \mathrm{kHz}$, the system transfer function, $H(\omega)$, can be 
approximated by

$$
H(\omega)=K \omega^{2},
$$

where $K$ is a constant. This confirms the two time derivative phenomenon expected from the total system. The transfer function block diagram is illustrated in figure 4.6.

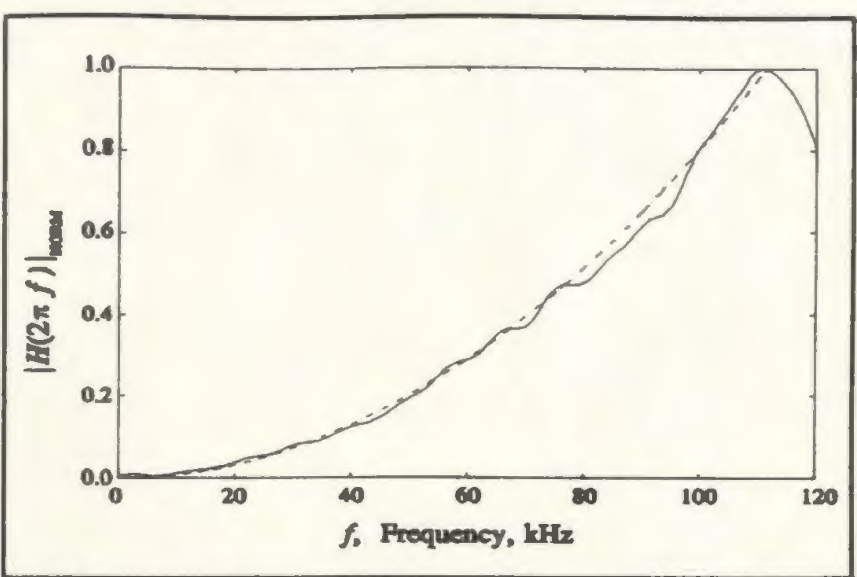

Figure 4.5: Magnitude Plot of the double hydrophone system transfer function, $H(\omega)$ (solid), and a least squares fitted second order polynomial, $K \omega^{2}$ (dotted).

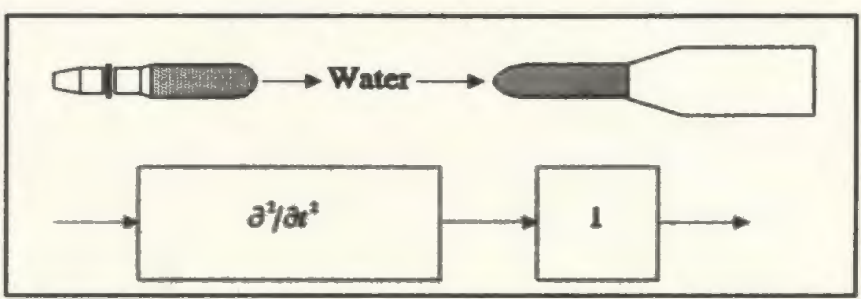

Figure 4.6: Hydrophone system response. allows us to reduce the number of elements in the transmit array by using discrete, equally spaced annular sections (c.f. figure 4.2). If the receiver hydrophone is placed along the $z$-axis $(\rho=0)$ then, assuming a flat band system transfer function, the backscattered signal may be reconstructed using the formula

$$
v_{\mathrm{MPS}}(r, t)=-\sum_{n=0}^{N} \varsigma_{n}(r, t) S(n)
$$

In (4.2), $v_{\text {MPS }}$ is the reconstructed backscattered signal, $\varsigma_{n}$ are the spherically reflected signals originating from each of the $N$ transmit elements, $\rho_{n}$ is the radial location of the $n^{t h}$ element, $\Delta \rho$ is the spacing between adjacent annular elements, $S(1)=\pi \Delta \rho^{2} / 4$ and $S(n)=\pi\left(\rho_{n}+1 / 2 \Delta \rho\right)^{2}-\pi\left(\rho_{n}-1 / 2 \Delta \rho\right)^{2}=2 \pi \rho_{n} \Delta \rho$. Thus a complete aperture can be synthesized 
by moving the transmit hydrophone to discrete positions along a single radius of the transmit aperture, corresponding to each discrete annular region. For these experiments, $N=30$ and $\Delta \rho=4 \mathrm{~mm}$, hence the aperture has a radius of $116 \mathrm{~mm}$.

In order to obtain the relationship between the reconstructed and predicted backscattered spectra, (4.2) should be weighted with the system transfer function, $H(\omega)$. Hence, if the MPS pulse is designed such that the effective maximum frequency, $f_{\max }$ (chapter 2) [Ziolkowski, 1991], of each driving function is below $110 \mathrm{kHz}$, then the backscattered spectrum, $\Lambda_{\mathrm{MPS}}$, and the Fourier transform of the reconstructed signal, $\Upsilon_{\mathrm{MPS}}$, are approximately related by

$$
\Upsilon_{\mathrm{MPS}}(r, \omega) \approx K \omega^{2} \Lambda_{\mathrm{MPS}}(r, \omega)
$$

in the range [0-120] $\mathrm{kHz}$. For the MPS parameters chosen, $f_{\max }=59.7 \mathrm{kHz}$.

\subsection{Direct Signal Cancellation}

The experiments discussed in this chapter are designed such that, theoretically, the direct and reflected signals do not overlap. However, practical experimentation showed that a small amount of residual direct signal overlapped the received signal, and in some cases completely overwhelmed the weak reflected signal. The residual direct signal is actually thought to be due to reflections from the conical shaped brass case that houses the receiver hydrophone. In order to remove this unwanted residual signal, a signal cancellation method is used. The residual plus spherically reflected signals are first measured by transmitting with the sphere present in the tank. The residual direct signal is then measured by transmitting with the sphere absent from the tank. The 
residual signal is thus removed from the residual/sphere signal by subtraction. The effectiveness of this method is illustrated in figures 4.7 and 4.8 on the following pages. Figure 4.7 shows the residual direct signal superimposed over the residual/sphere signal. The reflected signal is calculated to arrive at approximately $0.06 \mathrm{~ms}$. Figure 4.8 shows the reflected signal with the direct residual signal subtracted out. As expected, the reflected signal remains starting at approximately $0.06 \mathrm{~ms}$. Note here that the time axis on figures 4.7 and 4.8 are referenced to the start of waveform recording and not to the start of direct signal transmission.

\subsection{Background Noise}

The effect of background is reduced simply through signal averaging. The PC controller is capable of sending repeated signals through the system and averaging them. To demonstrate the effectiveness of this procedure, the background noise was measured by powering up the TX/RX amplifiers and

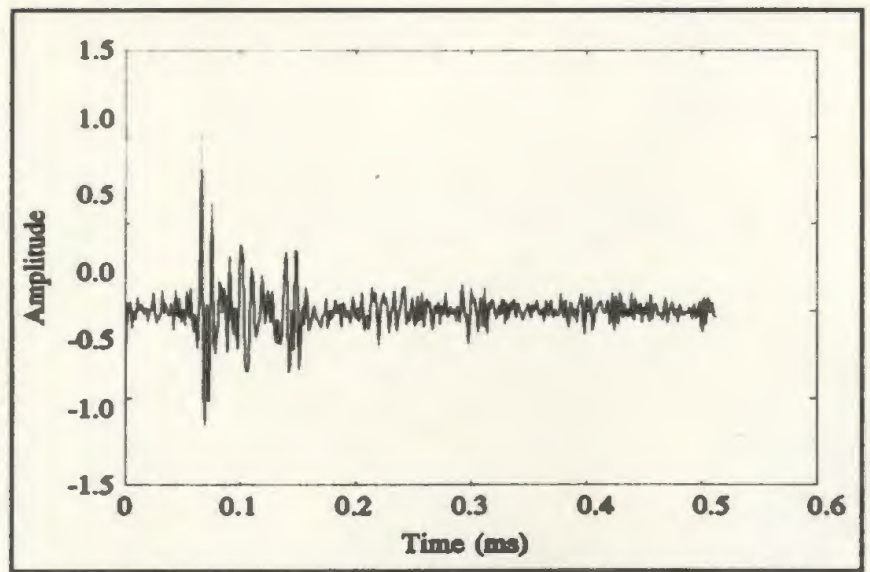

Figure 4.8: Spherically reflected signal obtained after the residual direct signal has been subtracted out. 
measuring the amount of random noise present. The noise signals were then averaged $M$ times to record the effective noise reduction. Figure 4.9 shows the effect of averaging 10 and 50 times. The FFT of the averaged signals shows a significant reduction of the noise power as the averaging is increased from 10 to 50 times. The ratio of signal energy between the $M_{1}=10$ and the $M_{2}=50$ signal is calculated using

$$
\frac{\mathscr{E}_{1}}{\mathscr{E}_{2}}=\frac{\sum_{l=0}^{N-1} n_{1}(l)}{\sum_{l=0}^{N-1} n_{2}(l)}
$$

where $\mathscr{E}$ is the energy, $n$ is the sample signal and $N$ is the number of samples taken. For this example, the ratio is 4.7642, which is close to the expected ratio for gaussian white noise (5.0). For

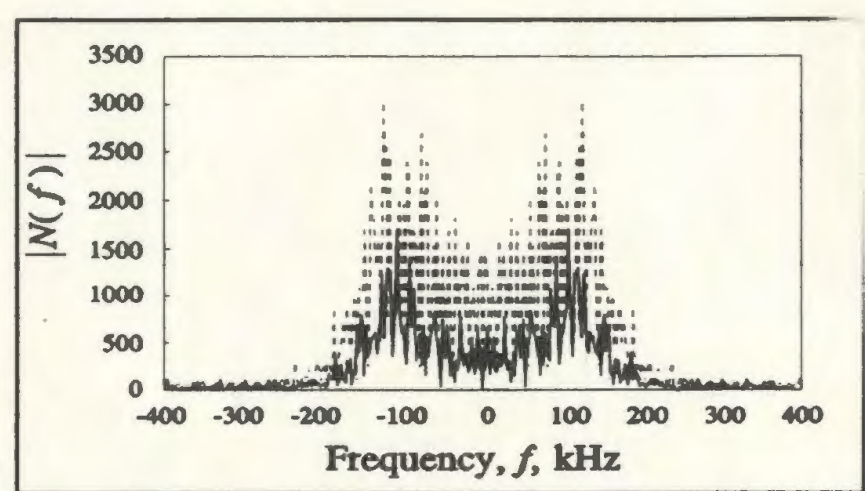

Figure 4.9: FFT of received background after 10 averages (dotted) and after 50 averages (solid)

these experiments, the signals were averaged 50 times each. A 512 point Hanning window was applied to the reconstructed sampled signals in order to smooth the backscattered signal FFTs. 


\section{Experimental Results}

In the figures which follow, the reconstructed backscattered, $\Upsilon_{\text {MPS }}$ spectra are compared to the predicted backscattered spectra, $\Lambda_{\text {MPs }}$. The predicted spectra has been weighted with the system transfer function, $H(\omega)$, in order to account for two time derivatives. In figures 5.1-5.3, the predicted versus measured spectra is shown for steel sphere radii 20,40 and $60 \mathrm{~mm}$ respectively for $r=15 \mathrm{~cm}$. In figure 5.4 , the predicted versus measured spectrum is shown for the $40 \mathrm{~mm}$ radius aluminum sphere for $r=15 \mathrm{~cm}$. Other plots of

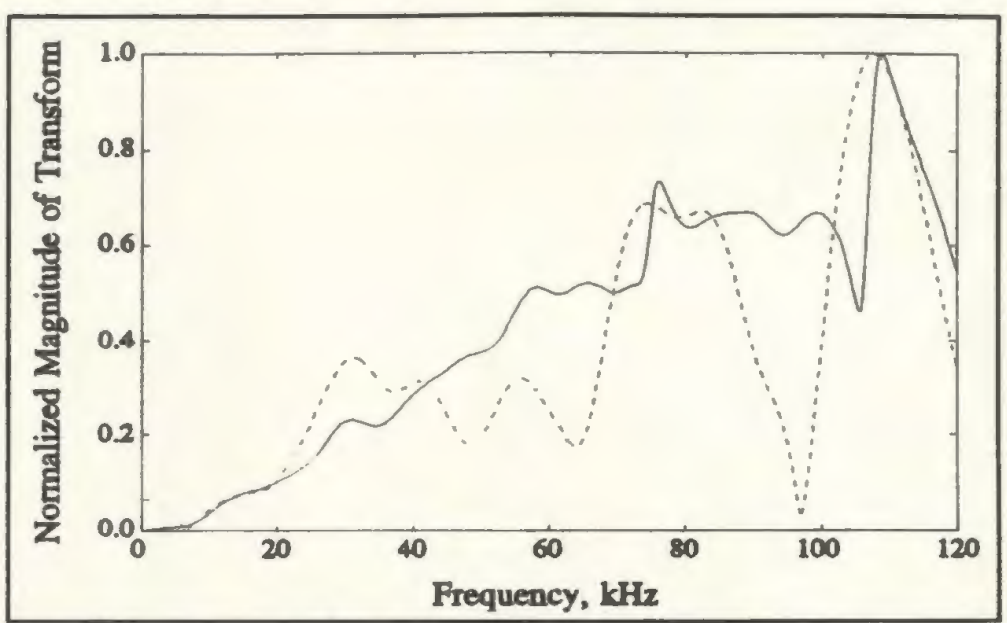

Figure 5.1: Comparison of $\left|\Upsilon_{\text {MPS }}\right|$ (dotted) with $\left|H \Lambda_{\text {MPS }}\right|$ (solid) for a $30 \mathrm{~mm}$ radius steel sphere at $r=150 \mathrm{~mm}$ from the array.

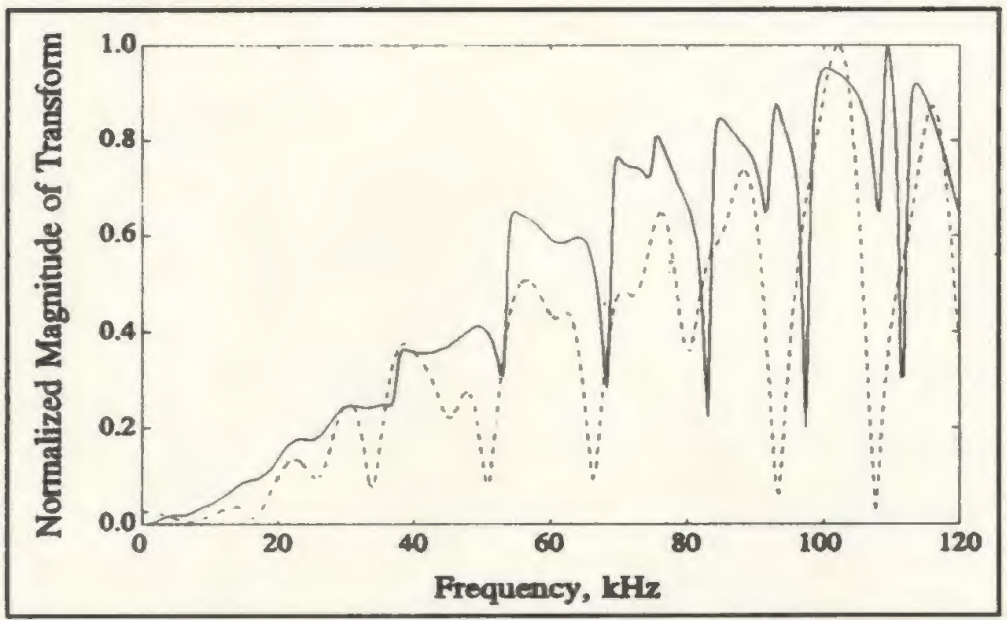

Figure 5.2: Comparison of $\left|\Upsilon_{\text {MPS }}\right|$ (dotted) with $\left|H \Lambda_{\text {MPS }}\right|$ (solid) for a $40 \mathrm{~mm}$ radius steel sphere at $r=150 \mathrm{~mm}$ from the array. measured backscattered spectra for the cases $r=25 \mathrm{~cm}$ and $r=35 \mathrm{~cm}$ are contained in appendix C. In all cases, both the predicted and measured spectra contain dips. Although the locations of the dips are different in the measured and predicted spectra, the average spacing between the dips are 
approximately the same. The differences between both spectra may be due to the following:

- An approximation to the MPS pulse is launched from the array. As such the measured backscattered spectra will also be an approximation.

- The material parameters used to generate the predicted spectra may not be the actual parameters of the spheres used in the experiments. Since accurate measurements of

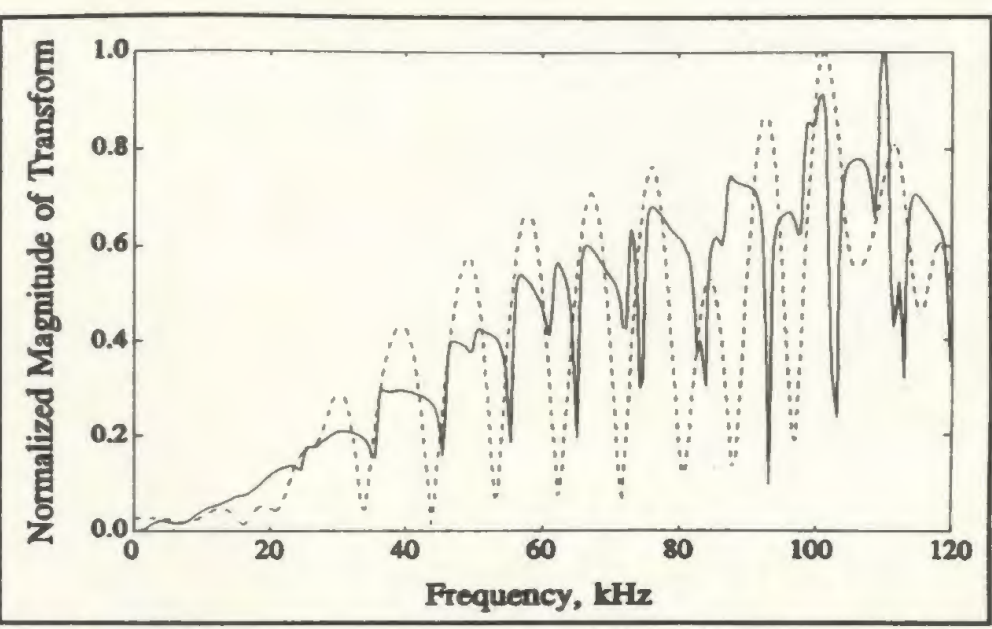

Figure 5.3: Comparison of $\left|\Upsilon_{\text {MPS }}\right|$ (dotted) with $\left|H \Lambda_{\text {MPS }}\right|$ (solid) for a $60 \mathrm{~mm}$ radius steel sphere at $r=150 \mathrm{~mm}$ from the array.

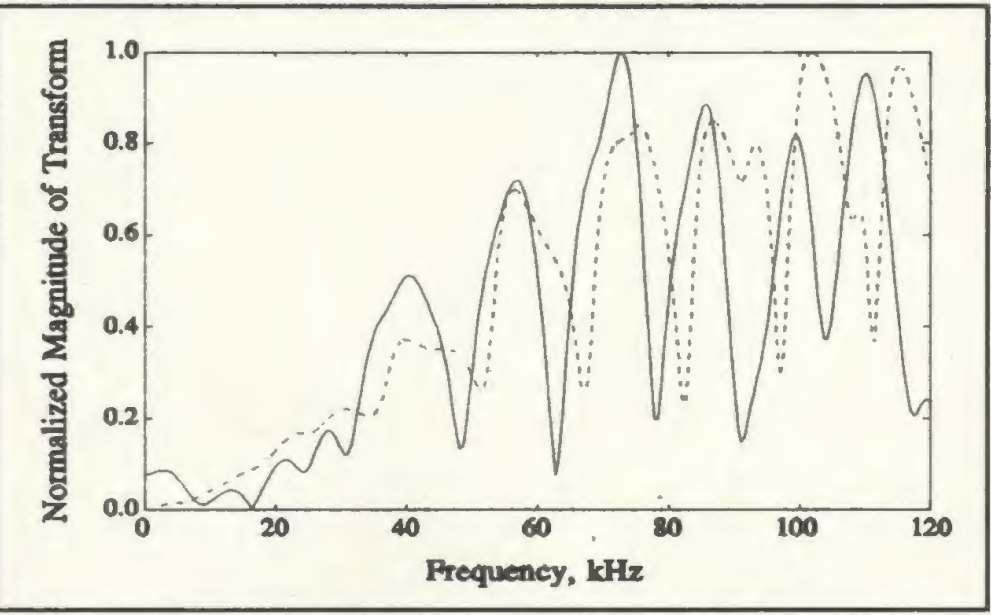

Figure 5.4: Comparison of $\left|\Upsilon_{\text {MPS }}\right|$ (dotted) with $\left|H \Lambda_{\text {MPS }}\right|$ (solid) for a $40 \mathrm{~mm}$ radius aluminum sphere at $r=150 \mathrm{~mm}$ from the array.

the material parameters of

the steel and aluminum were not available, estimates of $c_{e}$ and $\rho_{e}$ were made based on the grade of steel and aluminum used.

- The receiver hydrophone was slightly offset from the $z$-axis. Although it was hoped that this offset would not greatly affect the results, it may have contributed to the difference in locations of the dips in the measured versus predicted spectra. 
As was discussed in the previous chapter, a window function was applied to the measured reconstructed signals to impart spectral smoothing. It was determined that this window function has little effect on the location of the dips of the predicted spectra.

As was discussed in chapter 3 , the frequency of the dips in the backscattered spectra may be used to determine the size of the sphere. In this case, the average spectral spacing of the dips will be used, since they are consistent between measured and

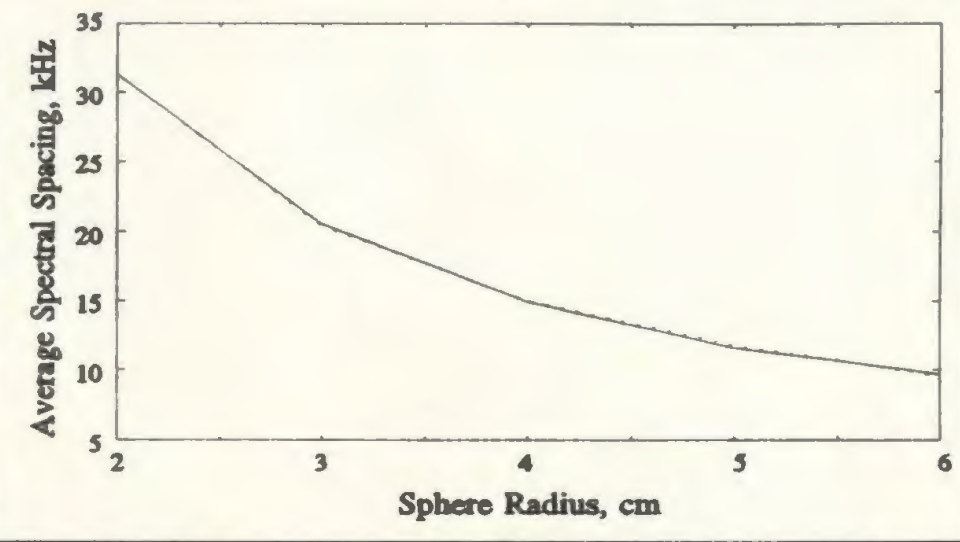

Figure 5.5: Average spectral spacing plot for steel spheres. Graph shows both extracted (solid) and modelled (dashed) data.

predicted spectra. The spectral dips of five steel sphere spectra $(2-6 \mathrm{~cm}$ radii) were located and the differences between adjacent dips were calculated and averaged. Since the dips seem to be most prominent between $30 \mathrm{kHz}$ and $120 \mathrm{kHz}$, only these dips were used in the calculation. The results are shown in figure 5.5 as the solid line. Since the curve appears to exhibit an inverse relationship, the extracted points were fitted with a $K_{1} / a+K_{2}$ curve via the least squares approach. The fitted curve is shown as the dashed line in figure 5.5. The maximum error in the fitted curve is $0.61 \%$ for points shown in the figure (radii of $2-6 \mathrm{~cm}$ ).

An identical analysis was performed on a set of aluminum sphere predicted backscattered spectra. As shown in figure 5.6, the modelled curve (dashed) agrees well with the predicted results (solid) with a maximum error of 1.65 percent for the points 
shown in the figure (radii of 2$6 \mathrm{~cm})$.

From the results above, it is seen that a method to extract the radius from a backscattered spectrum may be readily formulated. Using a number of spheres of known size, one may

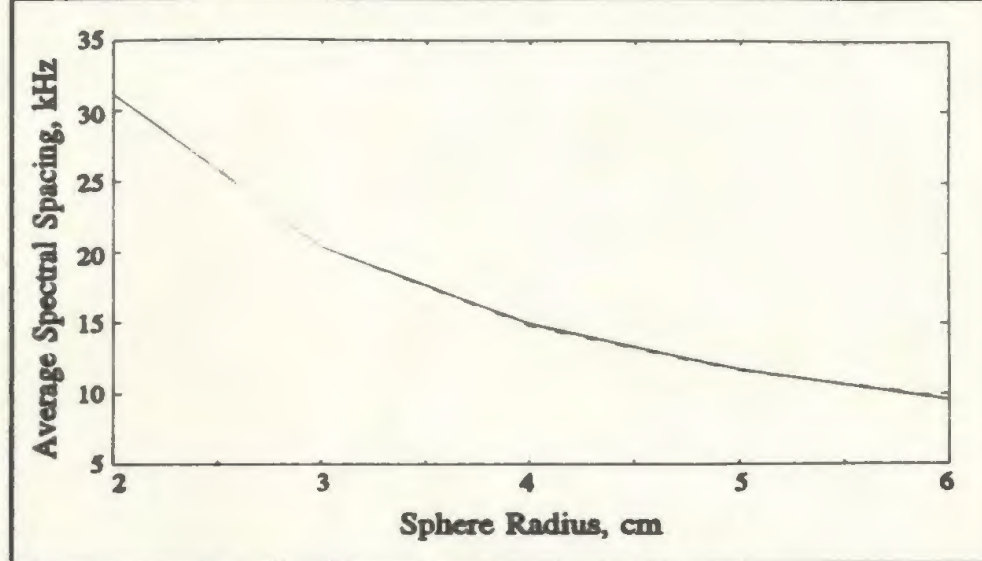

Figure 5.6: Average spectral spacing plot for aluminum spheres. Graph shows both extracted (solid) and modelled (dashed) data.

determine the constants in the $K_{1} / a+K_{2}$ curve from the dips in the backscattered spectra.

This calibration eliminates the need to determine $c_{e}$ or $\rho_{e}$ of the spheres. In order to extract the radius of an unknown sphere, one may simply examine the dips of its backscattered spectra and extract the sphere radius from the calibrated curve, $K_{1} / a+K_{2}$.

This method was tested on the measured backscattered spectra of the steel and aluminum spheres. The constants $K_{1}$ and $K_{2}$ were determined using the three steel sphere spectra obtained at $r=150 \mathrm{~mm}$. In order to automate the extraction procedure, a simple algorithm was formulated to determine the locations of the spectral dips. This algorithm numerically differentiates the input spectrum to locate where the slope of the spectrum changes abruptly. The algorithm was implemented as a MatLab function and is listed in appendix B. Table II shows the extraction results for each of the spheres at the indicated distances. The maximum error is $3.5 \%$ for the steel spheres and $6.5 \%$ for the aluminum sphere. The percent error for the aluminum sphere is somewhat higher than

${ }^{2}$ MatLab is ${ }^{\circ}$ copywrite 1984-1993 The Mathworks Inc. 
that of the steel spheres. This is to be expected since the modelled curve was fitted using the steel sphere spectra. However, the results are still favourable since the error seems to be within acceptable limits.

\begin{tabular}{|r||c||c|c||}
\hline $\begin{array}{c}\text { Actual } \\
\text { Size (cm) }\end{array}$ & $\begin{array}{c}\text { Dist. from } \\
\text { Array (cm) }\end{array}$ & $\begin{array}{c}\text { Estimated } \\
\text { Size (cm) }\end{array}$ & $\begin{array}{c}\text { Percent } \\
\text { Error }\end{array}$ \\
\hline \hline \multicolumn{4}{|c|}{ Steel } \\
\hline \hline 2.0 & 15 & 1.9941 & 0.3 \\
\hline 2.0 & 25 & 2.0044 & 0.2 \\
\hline 2.0 & 35 & 1.9975 & 0.1 \\
\hline 4.0 & 15 & 4.0968 & 2.4 \\
\hline 4.0 & 25 & 4.0939 & 2.3 \\
\hline 4.0 & 35 & 4.1403 & 3.5 \\
\hline 6.0 & 15 & 5.8445 & 2.6 \\
\hline 6.0 & 25 & 5.7954 & 3.4 \\
\hline 6.0 & 35 & 5.8632 & 2.3 \\
\hline \multicolumn{5}{|c|}{ Aluminum } \\
\hline 4.0 & 25 & 4.1773 & 4.4 \\
\hline 4.0 & 35 & 4.2610 & 6.5 \\
\hline \hline
\end{tabular}

Table I: Actual Vs. Extracted Sphere Radius 


\section{Qualitative Analysis of Two Ball Scattering}

In chapter three, the predicted MPS backscatter was derived and supported with the experimental spectra presented in chapter five. In this chapter, experimentally obtained two ball scattering spectra will be presented. The experimental setup used to obtain the data

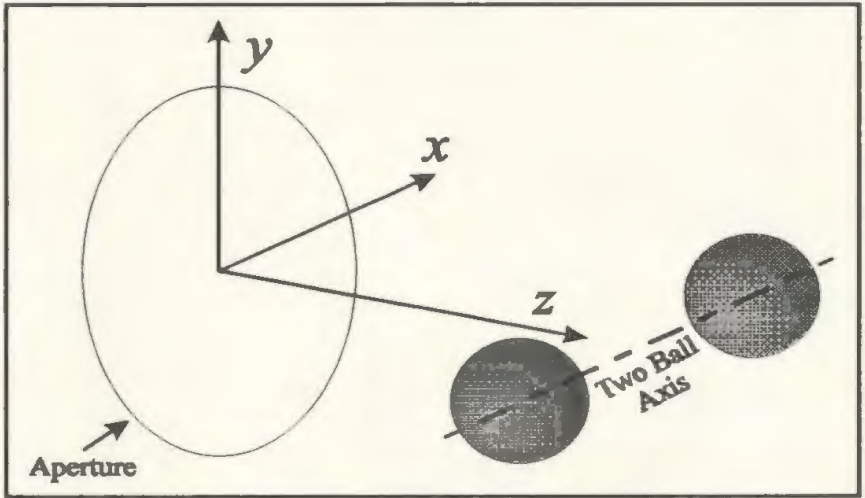

Figure 6.1: Placement of two spheres in from of acoustic array.

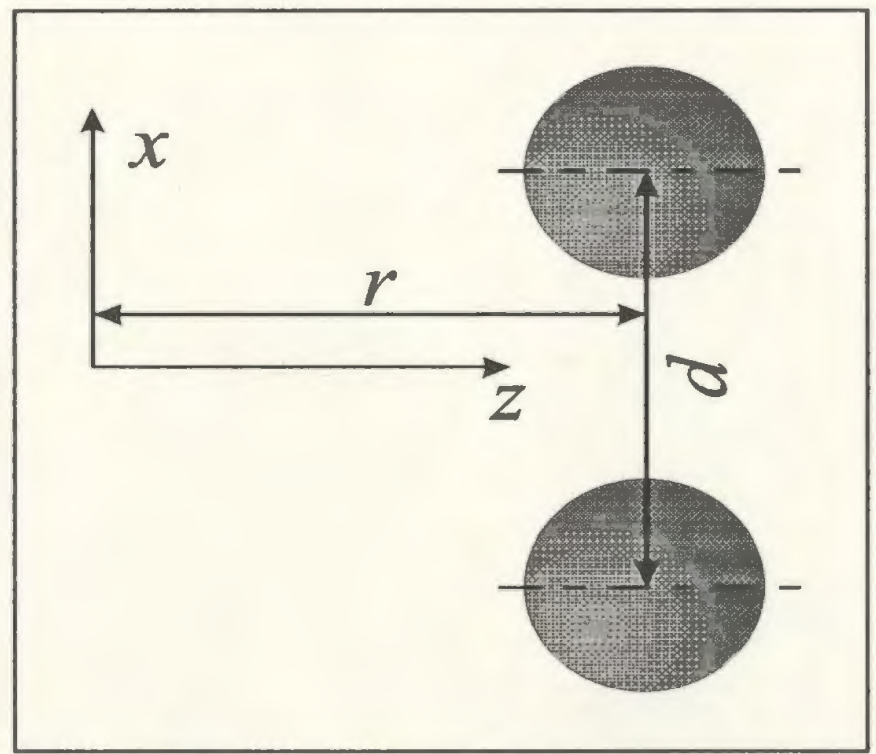

Figure 6.2: Two spheres, whose centres are separated by distance $d$, are placed at distance $r$ from the array. is somewhat different than that used in the single ball case. The symmetry of the single ball set up allowed the use of a single line of transducers to reconstruct the MPS backscatter. However, symmetry of the two-ball setup requires a complete half array to reconstruct the two ball backscatter (figure 6.1). The measured backscatter spectra obtained at $r=25 \mathrm{~cm}$ for $d=[4.0,8.0,14.0,20.0] \mathrm{cm}$ 
are illustrated in figures 6.3 to 6.6 respectively. Similar spectra obtained at $\mathrm{r}=35 \mathrm{~cm}$ are given in appendix $\mathbf{C}$.

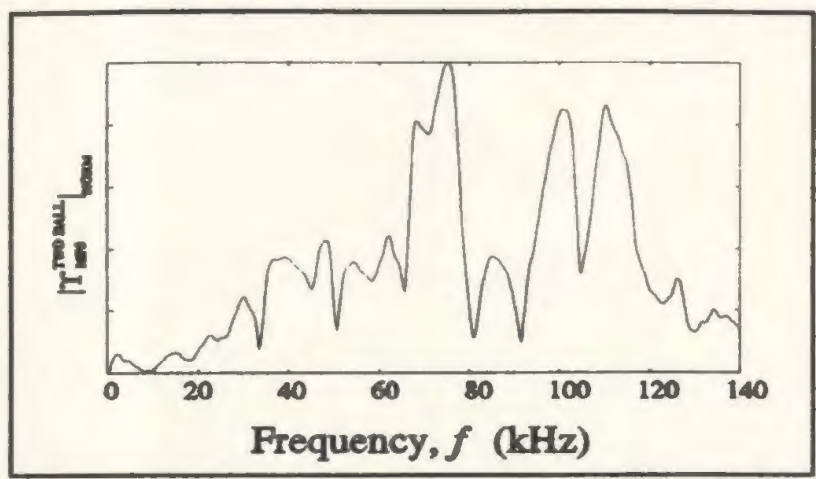

Figure 6.3: Two ball backscatter spectrum for $r=25 \mathrm{~cm}$ and $d=4 \mathrm{~cm}$.

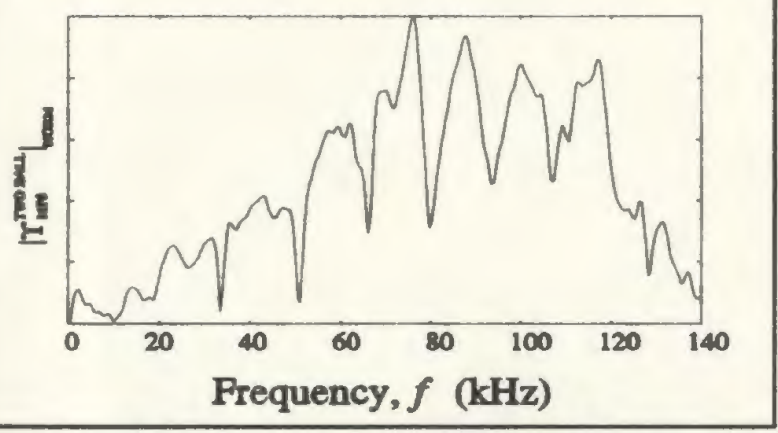

Figure 6.5: Two ball backscatter spectrum for $r=25 \mathrm{~cm}$ and $d=14 \mathrm{~cm}$.

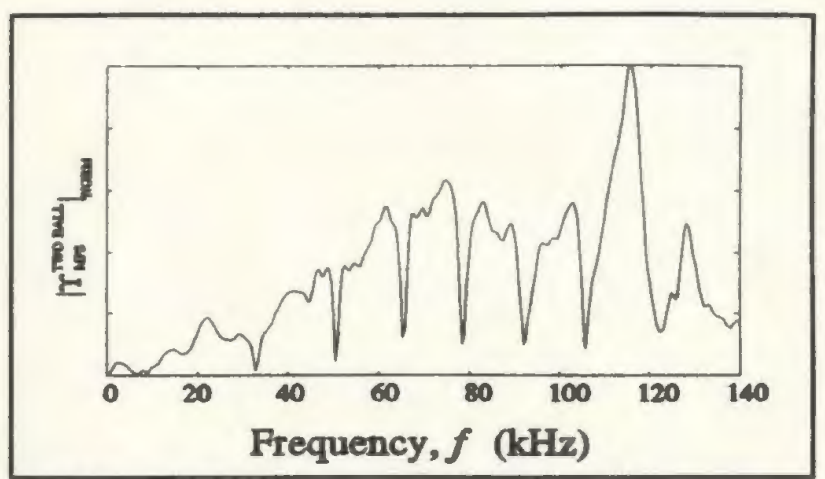

Figure 6.4: Two ball backscatter spectrum for $r=25 \mathrm{~cm}$ and $d=8 \mathrm{~cm}$.

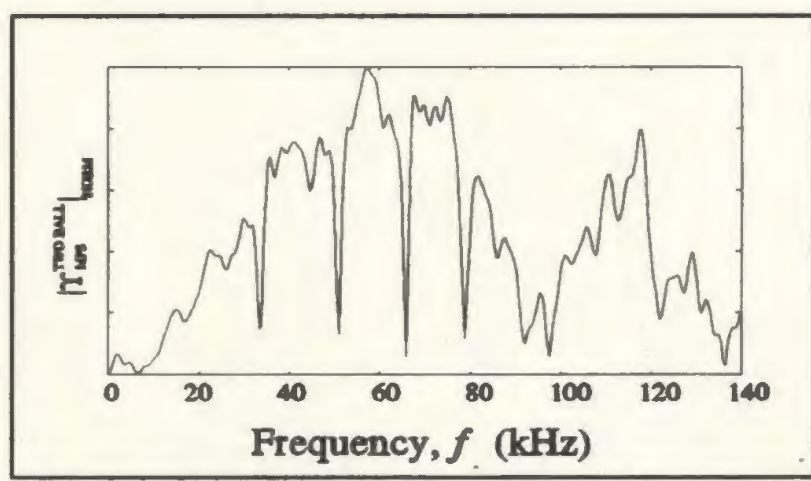

Figure 6.6: Two ball backscatter spectrum for $r=25 \mathrm{~cm}$ and $d=20 \mathrm{~cm}$.

As these figures illustrate, the backscattered spectra contain the characteristic frequency dips at locations similar to those of the single ball backscattered spectra. The dips also appear at spectral locations similar to those of the $40 \mathrm{~mm}$ radius single sphere backscattered spectrum, with slight differences.

The predicted backscattered spectrum may be obtained in a similar manner to the method outlined in chapter three. For instance, several authors have derived plane wave scattering from multiple spheres [Waterman \& Truell, 1961; Brunning and Lo, 1971; 
Peterson and Strom, 1973]. If the plane wave scattering expression is obtained, then it may be superposed over the plane wave decomposition of the LW; the result would be the two-sphere scattering expression. However, it may be difficult, if not impossible, to obtain the two ball LW scattering expression in closed form. As a first approximation, one may add the backscattered signals from two single spheres that are offset by angles of $\pm \theta$.

The practical significance of a two-ball backscattering expression is questionable due to its apparent complexity. For instance, the predicted spectra would only be valid for equal sized spheres with the same material parameters, with two-sphere axis perpendicular to that of the array. The analysis would not account for different sized spheres, spheres of differing materials, nor spheres whose axes are obliquely oriented to that of the array. In a practical sonar or radar system, one would launch a narrow waist LW in such a manner as to be able to discern single targets in a multi-target area using the single target backscatter expression. For instance, one may sweep an area using a pencil beam LW and obtain scattering signals from single targets if the targets are located far enough from one another to avoid spatial aliasing. In the case of closely spaced targets, the performance of a pencil beam LW system would depend on the size of the array and on the effective frequency of the LW pulse. Hence, the multiple sphere LW backscatter expression may not have any significance in a practical remote sensing system. However, further comments in this regard are deferred pending a complete analysis of the predicted two-sphere LW backscatter expression. This work will be completed at a later date. 


\section{Conclusions}

In the context of multi-time-derivative transmit-receive systems (such as that present in the forward propagating section of our experimental setup), the superior focusing properties of the MPS pulse over equivalent CW pulses has been demonstrated [6]. It thus seems natural to try to use the extended near-field characteristics of the MPS pulse, coupled with its broadband nature, to extract information from backscattered signals; the broadband nature of the signals allows for parameter extraction, while it is the enhanced propagation properties of the $\mathrm{LW}$ pulses that ensures that a relatively large amount of energy reaches the target. Other broadband signals might not have desirable propagation properties, and more focused monochromatic pulses would not have the desirable spectral width.

The sphere radii have been successfully extracted from the MPS backscattered spectra of several different sizes of steel and aluminum spheres, using a relatively naive algorithm. In this algorithm, the speed of sound in the sphere is assumed known (and is approximately the same for a wide variety of metals), and the radius is extracted from the spectrum. It may be possible to estimate both the material properties and the radius from the backscattered spectrum. Work on this is ongoing. 


\section{References}

Besieris, I. M., Shaarawi, A. M., \& Ziolkowski, R. W., "A bidirectional travelling plane wave representation of exact solutions of the scalar wave equation," $J$. Math. Phys., 30, 1254-1269 (1989).

Bowman, J. J., Senior, T. B. A., Uslenghi, P. L. E., "Electromagnetic and Acoustical Scattering by Simple Shapes," (North-Holland Publishing Company: Amsterdam), 1969.

Brittingham, J. B., "Focus Wave Modes in homogeneous Maxwell's equations: transverse electric mode," J. Appl. Phys., 54, 1179-1189 (1983).

Brüel and Kjær, "Instruction Manual, Hydrophones Types 8101, 8103, 8104, 8105," 1986.

Brunning, J. H., \& Lo, Y. T., "Multiple scattering of EM waves by spheres, part I and II," IEEE Trans. Ant. Prop., 19, 378-400 (1971).

Donnelly, R. K., \& Ziolkowski, R. W., "Designing Localized Waves," Proc. $R$. Soc. Lond. A, 440, 541-565 (1993).

Donnelly, R. K., \& R. W. Ziolkowski, R. W., "A method of constructing solutions of homogeneous partial differential equations: localized waves," Proc. $R$. Soc. Lond. A, 437, 673-692, (1992).

Donnelly, R., Power, D., Templeman, G., \& Whalen, A., "Graphical Simulation of Superluminal Acoustic Localized Wave Pulses," IEEE Trans. Ultrason., Ferroelec, Freq. Contr., To be published (1993).

Peterson, B., \& Strom, S., "T-Matrix for electromagnetic scattering from an arbitrary number of scatterers and representation of E(3)," Phys. Rev. D., 8, 3667-3677 (1973).

Power, D., Donnelly, R., \& Macisaac, R., "Spherical scattering of superpositions of localized waves," Phys. Rev. E. 8, 1410-1417 (1993).

Gray, D., ed., "American Institute of Physics Handbook," (McGraw Hill: New York), 1972.

Jones, D. S., "The Theory of Electromagnetism," (Pergamon: New York), 1964.

Lu, J., \& Greenleaf, J. F., "Experimental verification of nondiffracting X waves," IEEE Trans. Ultrason., Ferroelec, Freq. Contr., 39, 441-446 (1992). 
Morse, P., \& Ingard, K. U., "Theoretical Acoustics," (McGraw Hill: New York), 1968.

Overfelt, P. L., "Bessel-Gauss pulses," Phys. Rev. A, 44, 3941 (1991).

Reson System APS, "TC 4014 Reference Hydrophone," 1988.

Ristic, V., "Principles of Acoustic Devices," (Wiley: New York), 1983.

Waterman, P. C., \& Truell, R., "Multiple scattering of waves," J. Math. Phys., 2, 700734 (1961).

Wyld, H. W., "Mathematical Methods for Physics," (W. A. Benjamin Inc: Mass), 1976.

Ziolkowski, R. W, Lewis, D. K., \& Cook, B, D., "Evidence of localized wave transmission," Phys. Rev. Lett., 62, 147-150 (1988).

Ziolkowski, R. W., "Localized transmission of electro-magnetic energy," Phys. Rev. A, 39, 2005-2032 (1989).

Ziolkowski, R. W., \& Lewis, D. K., "Verification of localized wave transmission effect," J. Appl. Phys., 68, 6083-6086 (1990).

Ziolkowski, R. W., "Localized wave physics and engineering," Phys. Rev. A, 44, 39603984 (1991).

Ziolkowski, R. W., "Exact solutions of the wave equation with complex source locations," J. Math. Phys., 26, 861-865, (1985). 


\section{Appendix A: HP 9000 Controller Code}

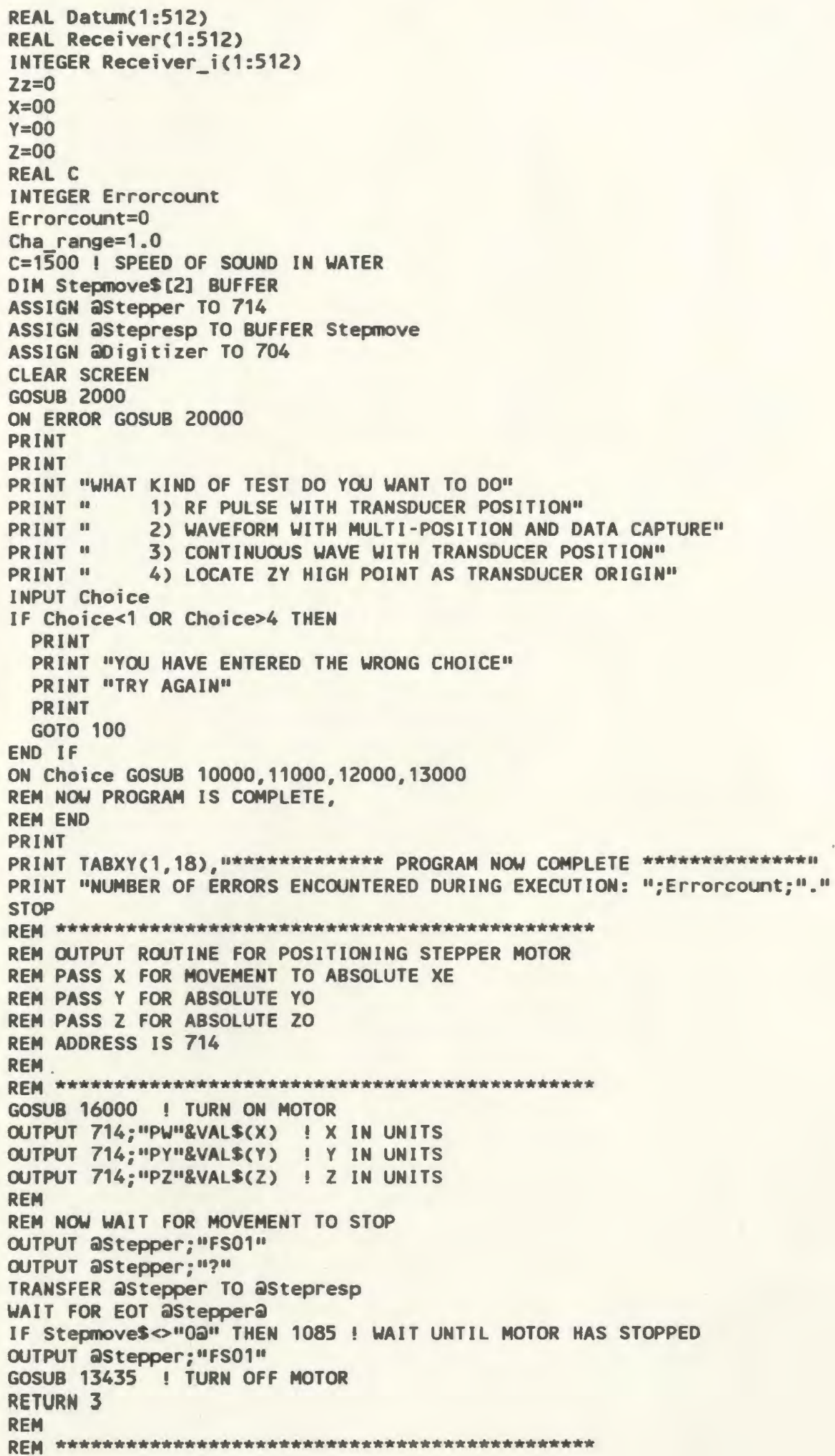


2001 REM SUBROUT INE TO RESET STEPPER MOTOR CONTROLLER

2010 REM SETS STEP RATE AT 500 STEPS PER SECOND

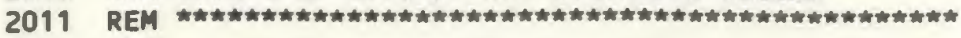

2012 REM

2020 OUTPUT $714 ; " A Z "$

2030 OUTPUT $714 ; "$ "AY"

2035 OUTPUT 714 ;"AW"

2040 OUTPUT 714;"R2500"

2050 OUTPUT 714;"RY500"

2055 OUTPUT 714;"RH500"

2060 RETURN

2070 REM

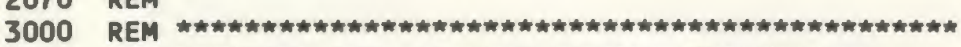

3001 REM POSITION THE DISTANCE BETWEEN TRANSDUCERS

3002 REM THIS IS THE $X$ AXIS

3010 REM

3020 INPUT "ENTER THE DISTANCE BETWEEN TRANSDUCERS IN CENTIMETERS $(<50) ", x$

3021 IF $x>50$ THEN

3022 PRINT

3023

3025

3027

3028

3029

3030

3032

3033

3034

3040

4000

4010

4020

4030

4040

4050

4060

4070

4080

4081

4082

4090

4100

4101

4110

4120

4130

4140

4150

4160

4165

4166

4170

4180

4190

4200

4210

4220

4230

4240

4250

4260

4270

4280

4290

4300

4301

4302 OUTPUT 704 ;"LV ",2

PRINT "PARAMETER OUT OF RANGE"

PRINT "TRY AGAIN"

GOTO 3020

END IF

$z z=x / 100$ ! OBSERVATION $z$ CO-ORDINATE

INPUT "ENTER HOLDOFF BEFORE THE START OF RECEPTION $(\mathrm{cm})$ ", HO

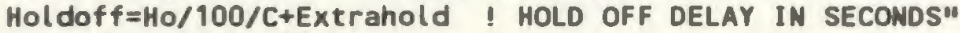

$X=-I N T(X \star 20000+.5) / 10$

GOSUB 1000

RETURN

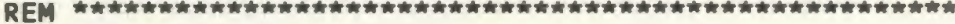

REM SET UP HP 5180A WAVEFORM RECORDER

REM THIS WILL BE SET UP TO TAKE 'SAMPLES' NUMBER OF SAMPLES

REM SPACED AT 1/'DFREQ' APARTK

REM CHANEL A HILL BE SELECTED IN dC COUPLING

REM BLOCK ONE UILL BE EXCLUSIVE MEMORY LOCATIONR

REM BLOCK SIZE WILL BE SET AT 'SAMPLES'

REM THE XYZ DISPLAY WILL BE ENABLED AS WELL IF A SCOPE IS

REM TO BE CONNECTED TO THE WAVEFORM ANALYSER

REM TRIGGER IS SET TO BE EXTERNAL FALLING EDGE

REM TRIGGER LEVEL IS SET AT 2 VOLTS WITH $100 \mathrm{mV}$ HYSTERISIS

REM POSITION OF TRIGGER HILL BE SET AT 'HOLDOFF'

REM LOCATION OF HP5180A IS AT HPIB LOCATION 4

REM VARIABLES (DFREQ, HOLDOFF, SAMPLES)

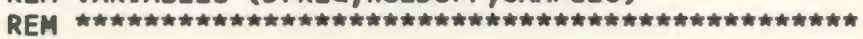

REM

REM SELECT CHANMEL

OUTPUT 704;"CH2 "

REM SELECT CHANNEL A RANGE (NOW SET TO 1)

OUTPUT 704: "BR ",Cha_range

REM SELECT dC COUPLIN̄G FOR CHANNEL A

OUTPUT 704: "BCO "

REM SELECT CHANNEL OFFSET (NOW SET TO ZERO)

OUTPUT 704 ; "BO ".

REM SELECT SWEEP MODE TO SINGLE SWEEP

OUTPUT 704: "SA1"

REM SELECT EXTERNAL SOURCE TRIGGER (VIA TRIGGER INPUT)

OUTPUT 704;"SE 1 "

REM SELECT POSITION OF TRIGGER AS SO MANY SECONDSN

OUTPUT 704; "PT", Holdoff

REM TIME BASE SELECT AS 1/DFREQ SECONDS BETWEEN SAMPLES

OUTPUT 704 : "IMM ",1/Dfreq

REM SPECIFY MEMORY RECORD LENGTH 'SAMPLES'

OUTPUT 704;"LE ".Samples

REM SELECT INTERNAL 20 MhZ CLOCK RATE

OUTPUT 704; "TEO "

4303 REM SELECT FALLING EDGE TRIGGERO 


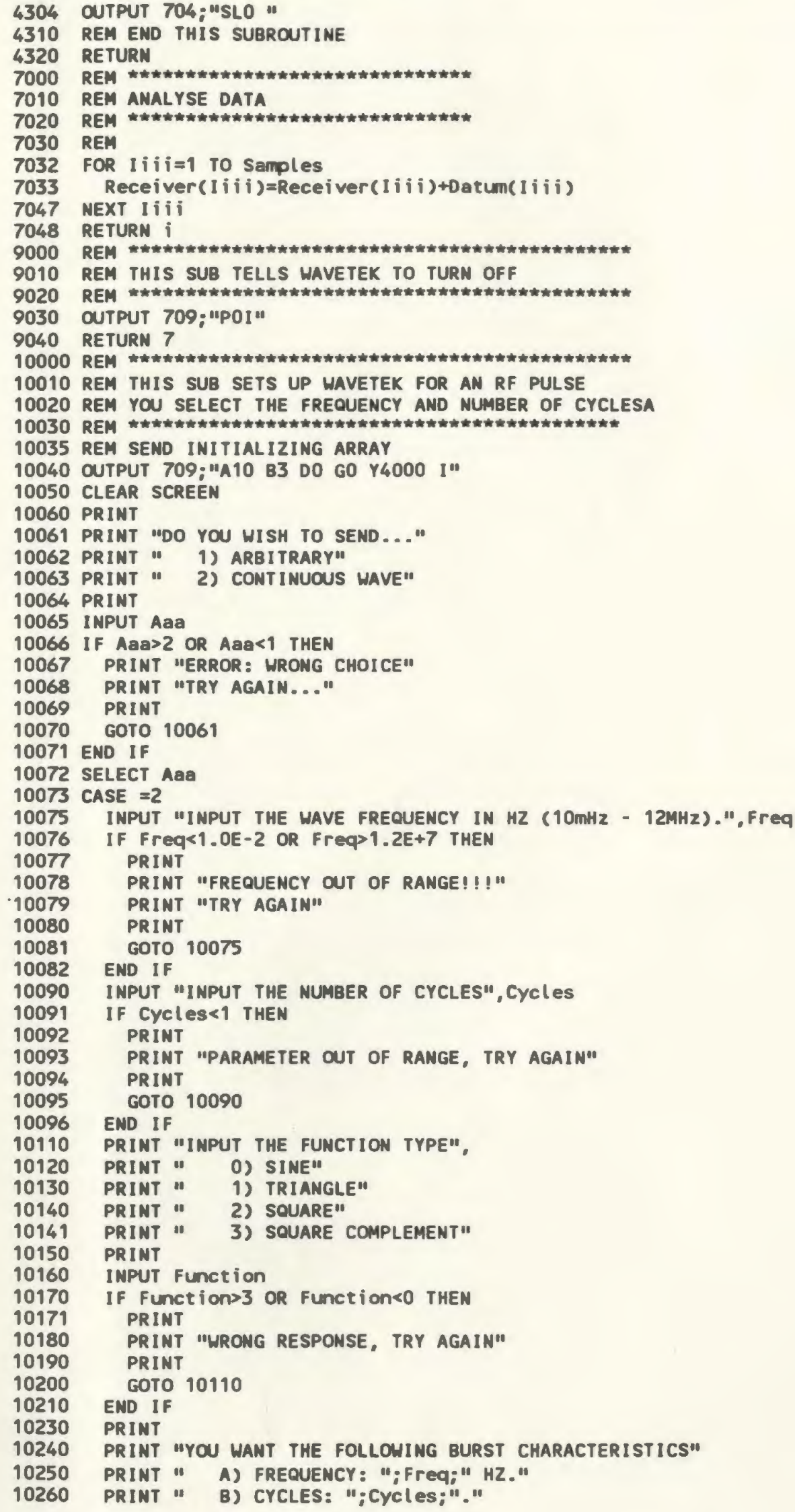




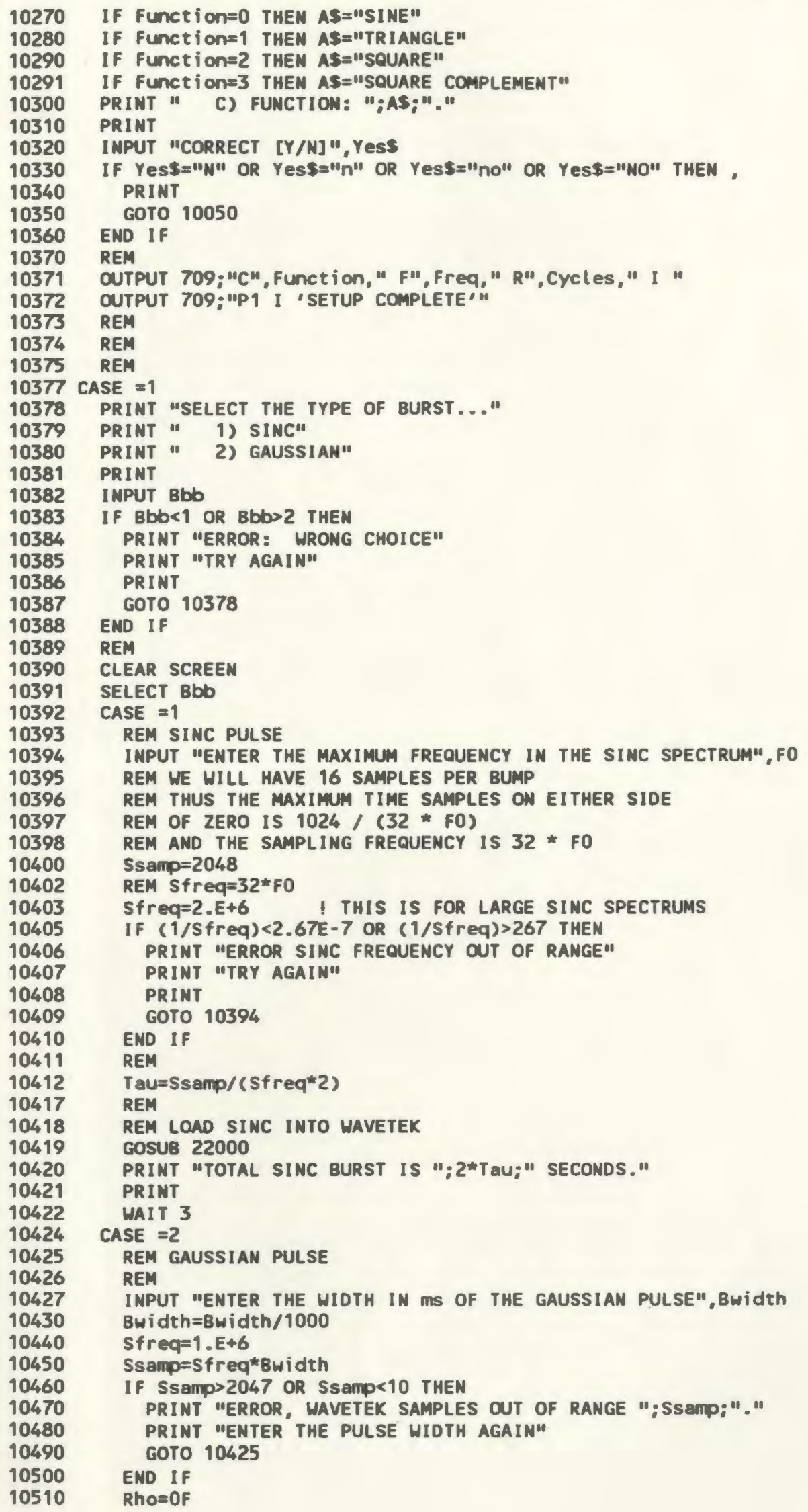




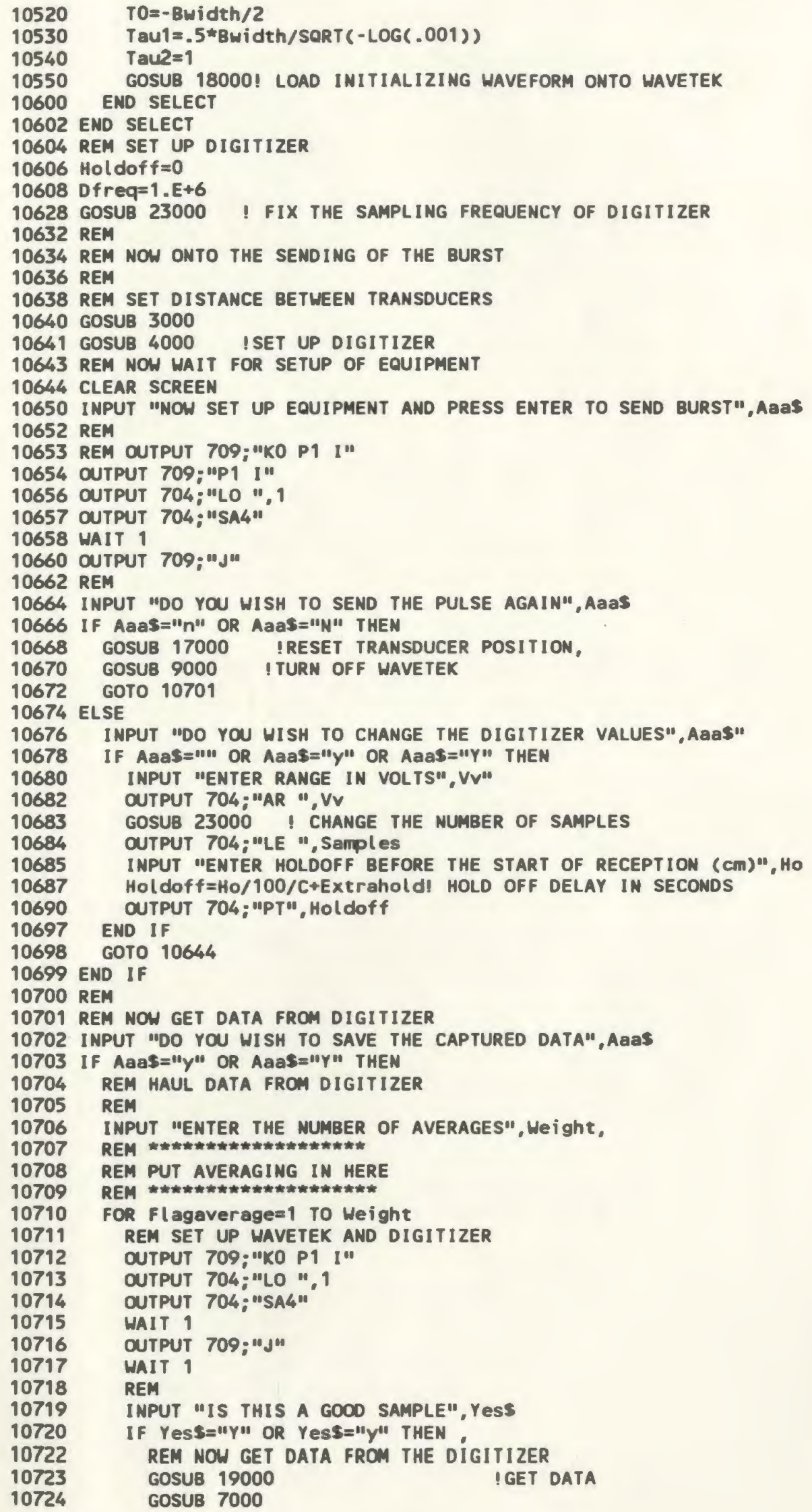




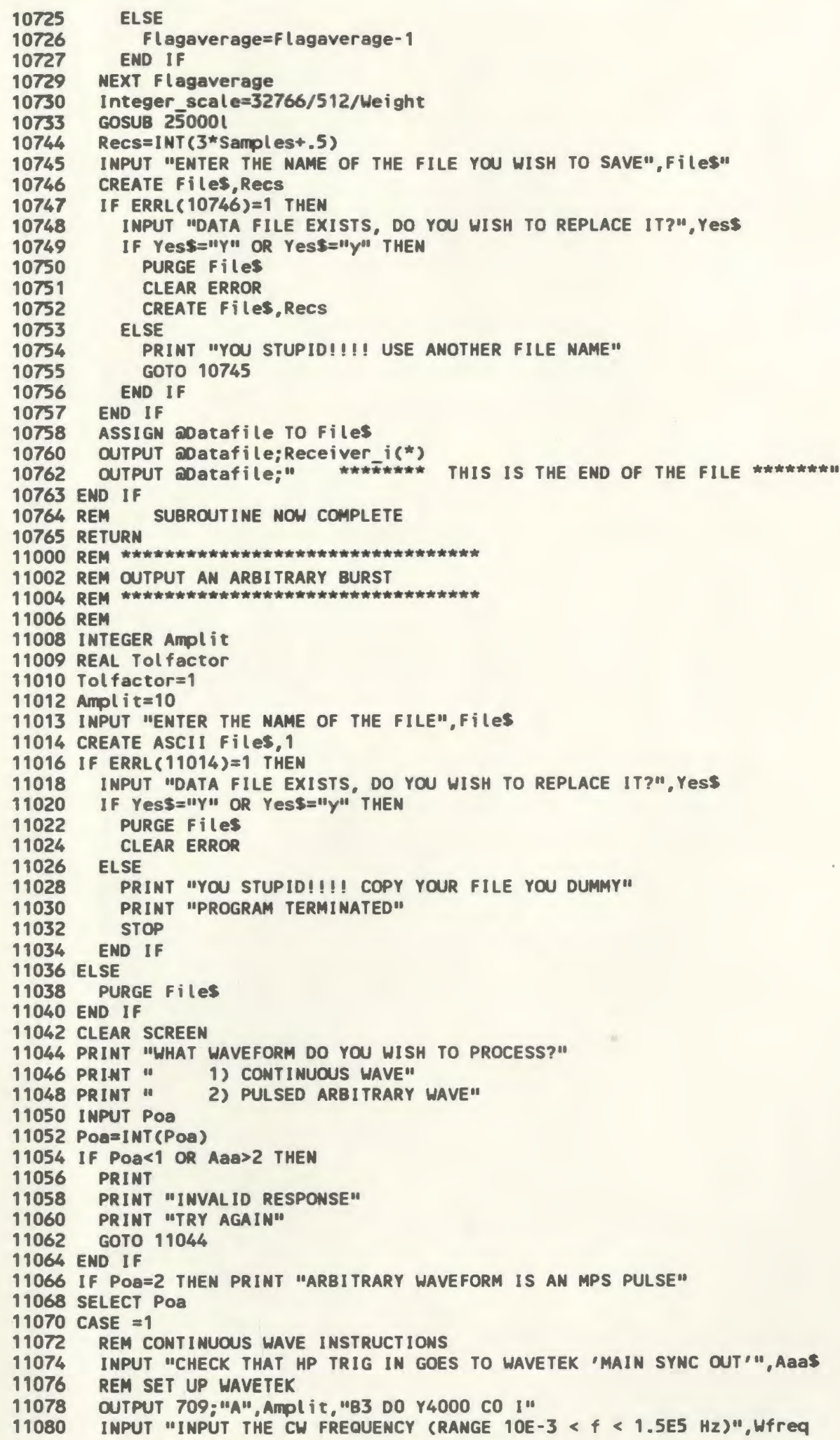


11082 If Wfreq<1.0E-2 OR Ufreq $>1.5 E+5$ THEN

11084 PRINT

11086 PRINT "WAVETEK FREQUENCY OUT OF RANGE ERROR"

11088 PRINT "INPUT FREQUENCY IN CORRECT RANGE"

11090 GOTO 11080

11092 END IF

11094 Msdcheck=1. OE -2

$11096 \quad F$ lag2 $=1$

11098 WHILE $F \log 2=1$

11100

11102

11104

11106

11108

11110

11112

11114

11116

11118

11120

11122

11124

11126

11128

11130

11132

11134

11136

11138

11140

11142

11144

11146

11148

11150

11152

11154

11156

11158

11160

11162

11164

11166

11168

11170

11172

11174

11176

11178

11180

11182

11183

11184

11185

11186

11187

11190

11191

11192

11193

11194

11196

11198

11200

Msd=Wfreq/Msdcheck

If Msd $=10$ THEN

Msdcheck=Msdcheck ${ }^{\star} 10$

ELSE

$F \log 2=0$

END IF

END UHILE

SELECT MSO

CASE 5 TO 10

Msd $=5$

CASE 2 TO 4.99999999999999

Msd $=2$

CASE 1 TO 1.99999999999999

Msd $=1$

END SELECT

Wf req=Msdcheck*Msd

PRINT "YOUR SELECTED FREQUENCY HAS BEEN ALTERED TO ";Hfreq; "Hz."

OUTPUT 709; "PO F", Wfreq, "I"

INTEGER NcYcle

INTEGER TCYcle

INTEGER Preamble

INPUT "ENTER THE MUMBER OF CYCLES YOU HISH TO SAMPLE" ,NCYCle

IF Ncycler1 OR Ncycle>5 THEN

PRINT "NUMBER OF CYCLES OUT OF RANGE"

PRINT "USE THE RANGE $[1,5] "$

GOTO 11142

END IF

INPUT "ENTER THE PREAMBLE CYCLES",Preamble

INPUT "ENTER THE TOTAL NUMBER OF CYCLES", TCYcle

IF Tcyclec(NcycletPreanble) THEN

PRINT "ERROR: YOU MUST ENTER A NUMBER GREATER THEN";NcYcle+Preamble GOTO 11154

END IF

OUTPUT 709;"R", Tcycle

Extrahold=Preamble/Wfreq

Btime=Ncycle/Wfreq

! SAMPLE NCYCLE CYCLES

CASE $=2$

INPUT "CHECK THAT HP TRIG IN GOES TO WAVETEK 'ARB SYNC OUT'",ABQS

REM BURST PARAMETERS

REM

REM CALCULATE hAVETEK PARAMETERS

REM S\$\$\$\$\$\$\$\$SSSS\$SS\$S\$\$SS\$\$\$\$\$\$\$\$\$\$\$\$\$\$\$\$\$\$\$\$\$

REM SSSS\$SS\$S\$\$\$S\$S\$\$\$\$S\$\$\$\$\$\$\$\$\$\$\$\$\$\$\$\$\$\$\$\$\$\$

REM SSS CHANGE ARBITRARY HP PARAMS HERE \$\$\$

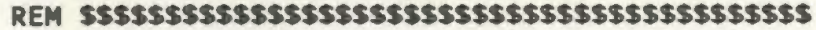

REM SSSS\$S\$\$S\$S\$\$S\$S\$\$\$\$\$SS\$\$\$\$\$\$\$\$\$\$\$\$\$S

Extrahold $=0$

Ssamp $=200$

Sfreq $=2 . E+6$

Rho $=0$

REM

Bt ime=Ssamp/sfreq

Dfreq $=1 . E+6$

REM NOU SET UP THE HAVEFORM RECORDER

11204 Sampl et ime=8 t ime $2_{2.0}$

11208 Shortsamples=Dfreq ${ }^{\star}$ Samplet ime

11210 Samples=2^(INT(LGT(Shortsamples)/LGT(2)+1))

11212 if Samples $<512$ THEN Samples $=512$ ) 
11214 PRINT "NUMBER OF SAMPLES HILL BE ";Samples;"."

11215 PRINT "REMEMBER TO CHANGE THE DIMENSIONING OF DATUM AND RECEIVER"

11216 PRINT "TO RELECT THIS NUMBER"

11218 WAIT 2

11219 REM NOW GET READY FOR CALIBRATION

11220 CLEAR SCREEN

11222 PRINT "YOU MAY NOW CALIBRATE YOUR RECEIVER PRE-AMP"

11224 PRINT "AND TRANSMITTER PONER AMP POWER SETTINGS."

11226 PRINT "YOU CAN NOW ENTER THE TRANSDUCER SEPARATION FOR YOUR CALIBRATION"

11228 GOSUB 3000 I POSITION $X$

11231 IF $P O a=2$ THEN

$11232 \mathrm{Rn}=22$

11235 GOSUB 21000

ILOAD INITIALIZING WAVEFORM ONTO WAVETEK

11236 END IF

11239 GOSUB 4000

11240 OUTPUT 709;"KO P1 ["

11241 OUTPUT 704:"LO ", 1

SET ARB ADDRESS TO 0

11242 OUTPUT $704 ; "$ "SA4"

11243 WAIT 1

11244 OUTPUT $709 ; " J "$

11245 WAIT 1

11246 Weight $=1$

11248 GOSUB 19000 IGET MEAN AND VARIANCE

11249 CLEAR SCREEN

11250 PRINT "THE MEAN IS ";Meany

11251 PRINT "THE VARIANCE IS ":VarYN

11252 Vartol=VarYV

11254 INPUT "DO YOU HISH TO RE-SEND THE BURST FOR NEW CALIBRATION [Y/N].", YES\$

11255 If Yes\$="Y" OR Yes\$="Y" OR Yes\$="YES" OR Yes\$="Yes" OR YeS\$="Yes" OR YeS\$=" THEN

11256 REM PRINT "THE HAVETEK AMPLITUDE IS SET FOR";AMpl it; "V PEAK TO PEAK"

11257 PRINT "THE DIGITIZER IS SET FOR";Cha range;"V PEAK TO PEAK"

11258 INPUT "DO YOU HISH TO CHANGE THIS", Yes2S

11259 If Yes2\$="Y" OR Yes2\$="y" OR Yes2\$="YES" OR Yes2\$="yes" OR Yes2\$="Yes" THEN

11260 REM INPUT "ENTER THE AMPLITUDE BETHEEN 1 AND 10" AmplitR

11261 INPUT "ENTER THE PEAK RANGE IN VOLTS",Cha_range"

11262 REM IF Amplit $<1$ OR Amplit $>10$ THENL

11263 REM PRINT "INVALID RESPONSE, TRY AGAIN"

11264 REM GOTO 11256

11265 REM END IF

11266 REM OUTPUT 709;"A",AMplit

11267 GOSUB 4000

11269 END IF

11270 GOTO 11240

11272 END If

11274 REM

11275 REM SET THE VARIANCE TOLERANCE

11276 REM

11277 INPUT "ENTER THE TOLERANCE FACTOR FOR SEQUENCE REJECTION",TOlfactor"

11280 REM NOW ONTO THE TEST

11281 REM HE MUST NOW ASK QUESTIONS

11282 REM ABOUT THE SIZE OF THE ARRAY

11283 REM

11284 CLEAR SCREEN

11286 REM PRINT "YOU WILL NOW BE ASKED QUESTIONS "

11288 REM PRINT "ABOUT THE SIZE OF THE RECEIVING ARRAY "

11290 REM PRINT "THIS ARRAY HILL BE ROUND "I

11292 REM PRINT

11294 REM INTEGER Rside

11296 INTEGER TSIdes

11298 REM INPUT "ENTER THE MUMBER OF TRANSDUCERS ALONG A RADIUS", Rs ide

11300 REM INPUT "ENTER THE SPACING BETHEEN TRANDUCERS (cm)",Rspace

11302 REM IF Rside*Rspace>45 THEN

11304 REM PRINT "ERROR: YOU HAVE TOO MANY TRANSOUCERS OR TO A TOO LARGE SPACING"

11306 REM PRINT "RE-ENTER THE RECEIVER VALUES."

11308 REM GOTO 11298

11310 REM END IF

11322 REM PRINTF

11324 PRINT "YOU HILL NOW BE ASKED ABOUT THE TRANSMITTING ARRAY"

11326 INPUT "ENTER THE MUMBER OF TRANSDUCERS ALONG A RADIUS", Tside 
11328 INPUT "ENTER THE DISTANCE BETWEEN TRANSDUCERS $(\mathrm{cm}) "$ "Tspace

11331 IF Tside*Tspace>45 THEN

11332 PRINT "ERROR: YOU HAVE TOO MANY TRANSDUCERS OR TO A TOO LARGE SPACING"G

11334 PRINT "RE-ENTER THE TRANSMITTER VALUES."

11336 GOTO 11322

11338 END IF

11340 CLEAR SCREEN

11341 REM Rspace=Rspace/100

11342 REM DELETE THIS NEXT LINE IF YOU WANT MORE THAN ONE RECEIVER IN ARRAY

11343 Rspace $=0$

11344 Rside $=0$

$11346 \mathrm{~T}$ space=Tspace $/ 100$

11347 PRINT "YOU HAVE ENTERED THE FOLLOWING ARRAY PARAMETERS..."

11348 PRINT

11349 REM PRINT "RECEIVING ARRAY:"

11350 REM PRINT " A) TRANSDUCERS ALONG RADIUS: ";Rside

11351 REM PRINT " $\quad$ B) ELEMENT SPACING: "; Rspace;"m."

11354 REM PRINT

11356 PRINT "TRANSMITTING ARRAY"

11358 PRINT " D) TRANSDUCERS ALONG SIDE: "; Tside

11360 PRINT " E) ELEMENT SPACING: "; Tspace;"m."

11362 INPUT "IS THIS CORRECT [Y/n]",AaAS

11364 IF Aaas $[1 ; 1]=" N "$ OR Aaas $[1 ; 1]=" n "$ THEN 11284

11366 GOSUB 9000 ! TURN OFF WAVETEK

11368 REM

11370 CLEAR SCREEN

11375 Numtest=Tside

11376 CLEAR SCREEN

11381 PRINT "THERE WILL BE";NUntest;"POINTS TO PROCESS."

11382 REM

11383 REM BATTERY OF TESTS

11384 REM

11386 Recs=INT $\left(3.5^{\star}\right.$ Samol es *Tside+ .5)

11388 CREATE Files, Recs

11390 ASSIGN aDatafile TO Files; FORMAT OFF

11392 INPUT "ENTER THE NUMBER OF AVERAGES", Weight

11393 Integer scale $=32766 / 512 /$ Weight

11395 Badcount $=0$

11396 Badaver $=0$

11398 REM

11399 REM THIS IS HHERE THE OUTER LOOP STARTED

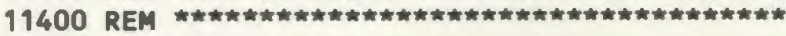

11401 REM FOR Rr=0 TO (Rside-1)*Rspace STEP Rspece

11402 REM

11403 REM I HAVE ARBITRARILY CHOSEN THE OBSERVATION RHO CO-ORDINATE

11404 REM AS BEING IN THE OBSERVATION X DIRECTION

11405 REM THIS IS JUST THE CONVENTION FOR THIS EXPERIMENT

11406 REM SO IF SOURCE $=\left(x^{\prime}, y^{\prime}, z^{\prime}\right)$ AND OBSERVATION $=(x, y, z)$

11407 REM THEN RR=SQRT $\left(x^{\wedge} 2+y^{\wedge} 2\right)^{\prime}$

11408 REM OUR 2 IS ACTUALLY $2 Z$

11409 REM $z^{\prime}$ SET AT ORIGIN O

11410 REM $y=0$ AND $x=R r=R H O$

11411 REM $X^{\prime}, Y^{\prime}=X X, Y Y$

11412 REM ALSO Rn CAN BA CALCULATED AS SQRT $\left(\left(x-x^{\prime}\right)^{\wedge} 2+\left(y-y^{\prime}\right)^{\wedge} 2+\left(z-z^{\prime}\right)^{\wedge} 2\right)$

11413 REM WE WILL BE CALCULATING RN AS

11414 REM AN APPROXIMATE AT THE OBSERVATION RHO

11415 REM EQUAL TO ZERO. IN THIS WAY

11416 REM THE WAVE WILL BE THE SAME

11417 REM REGARDLESS OF THE OBSERVATION CO-ORDINATES

11418 REM SO Rn=SQRT $\left(X x^{\wedge} 2+Y y^{\wedge} 2+Z z^{\wedge} 2\right)$

11419 FOR $X X=0$ TO Tspace*Tside STEP Tspace

11420 REM CALCULATE RHO HERE AND OUTPUT MPS

11421 REM THE NEXT LINE SHOULD CHANGE YY=0 TO $X X$ FOR SQUARE ARRAY)

11422 REM OTHER WISE ROUND ARRAY

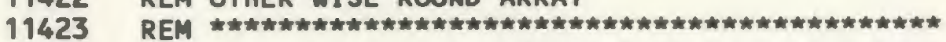

11424 REM THIS IS WHERE THE EXTRA LOOP STARTED BEFORE

11425 REM

11426 REM FOR $Y Y=0$ TO O STEP Tspace

11427 REM 
11428

11429

11430

11431

11432

11433

11434

11435

11436

11437

11438

11439

11440

11441

11442

11443

11444

11445

11446

11447

11448

11449

11450

11451

11452

11453

11454

11455

11456

11457

11458

11459

11460

11461

11462

11463

11464

11465

11466

11467

11468

11469

11470

11471

11472

11473

11474

11475

11477

11478

11479

11480

11481

11482

11483

11484

11485

11486

11487

11489

11490

11491

11492

11494

11495

11496

11497

11498

11499
REM NOTE THAT SIMCE $\times$ IS ARBITRARILY CHOSEN

REM AS THE DIRECTION ON WHICH THE

REM OBSERVATION RHO WILL LIE

REM THEN THERE IS SYMMETRY ABOUT THE

REM $x, x^{\prime}$ AXIS FOR THE SQUARE ARRAY

REM SO WE NEEO ONLY GO FROM O TO Yymax ON THE

REM TRANSMITTER SIDE

REM AND TAKE CARE OF THE REST IN THE DATA PROCESING

REM SECTION

REM

PRINT TABXY (1,3), "PROCESSING FOR RECEIVER SPACE ";Rr;" cm. "

PRINT TABXY $(10,4)$, "TRANSMITTER X SPACE "; XX;" cm." "

PRINT TABXY $(10,5)$, "TRANSMITTER Y SPACE ";YY;" cm. "

REM

REM Rn IS THE ABSOLUTE DISTANCE BETWEEN"

REM RECEIVER AND TRANSMITTER AT $x, y=0$

REM WHICH IS SQRT( $\left.x^{\prime \wedge} z+y^{\prime \wedge} z+\left(z-z^{\prime}\right)^{\wedge} 2\right)$

$R n=S O R T\left(X x^{\wedge} 2+Y Y^{\wedge} 2+Z z^{\wedge} 2\right)$

REM

REM MUST CALCULATE THE SOURCE CO-ORDINATEY

REM RHO WHICH IS SIMPLY SORT( ( $\left.\left.x^{\prime \wedge} 2+y^{\prime \wedge} 2\right)\right)$;

SThO=SQRT $\left(X X^{\wedge} 2+Y Y^{\wedge} 2\right)$ ! SOURCE CO-ORDINATE RHO

REM

REM $d x=$ TSPACE, $d y=T S P A C E$

REM WE MUST NOW CALCULATE THE RADIAL DISTANCE

REM BETWEEN THE TRANSMITTER AND RECEIVER

REM THE $Z$ DISTANCE (VARIABLE $X$ ) IS OF COURSE $Z Z$

REM THE PERPENDUCULAR RHO DISTANCE (VARIABLE $Z$ )

REM IS SQRT $\left(\left(x-x^{\prime}\right)^{\wedge} 2+\left(y-y^{\prime}\right)^{\wedge} 2\right)$

REM WHICH IS SQRT $\left((R r-X X)^{\wedge} 2+Y Y^{\wedge} 2\right)$

REM

Trho=SQRT $\left((R r-X X)^{\wedge} 2+(Y Y)^{\wedge} 2\right)$

REM

REM SO Trho IS THE PERPENDICULAR DISTANCE BETWEEN TRANSDUCERS"

$Z=-$ INT $(2000000$ *T rhot .5)/10

I SET STEPPER MOTOR 2 DISTANCE

GOSUB 1000

GOSUB 4000

GOSUB 21000

REM

REM DETERMINE THE VALUE OF THE VARIANCE FOR THIS EXPERIMENTN

REM

PRINT TABXY $(10,9)$, "DETERMINING THE VARIANCE FOR SAMPLE REJECTION

her aver $=60$

Vartol $\overline{2}=0$

F lagaverage $=10$

WHILE Flagaverage $<=$ Number_aver

REM SET UP WAVETEK AND DIGITIZER

OUTPUT 709:"KO PI I"

OUTPUT 704;"LO ",1

OUTPUT $704 ; "$ "SA4"

WAIT 1.5

OUTPUT $709 ; " \mathrm{Jn}$

WAIT 1

REM

REM NOW GET DATA FROM THE DIGITIZERR

GOSUB 19000

I GET DATA

PRINT TABXY $(10,8)$ "CURRENT SAMPLED VARIANCE IS";VarY

If Badsample_aver $=1$ THEN

Badaver $=$ Badaver +1

PRINT TABXY(1,13), "BAD VARIANCE SAMPLE NUMBER";Badaver;"HAS OCCURED" ELSE

Vartol 2=Vartol2+Vary/Number_aver

$F$ lagaverage $=F$ lagaverage +1

END If

END WHILE

Vartol $=$ Vartol 2

REM

PRINT TABXY $(10,9)$ " "VARIANCE FOR THIS AVERAGE SEqUENCE IS";Vartol;"

REM RESET THE VALUE OF RECEIVER ARRAY 


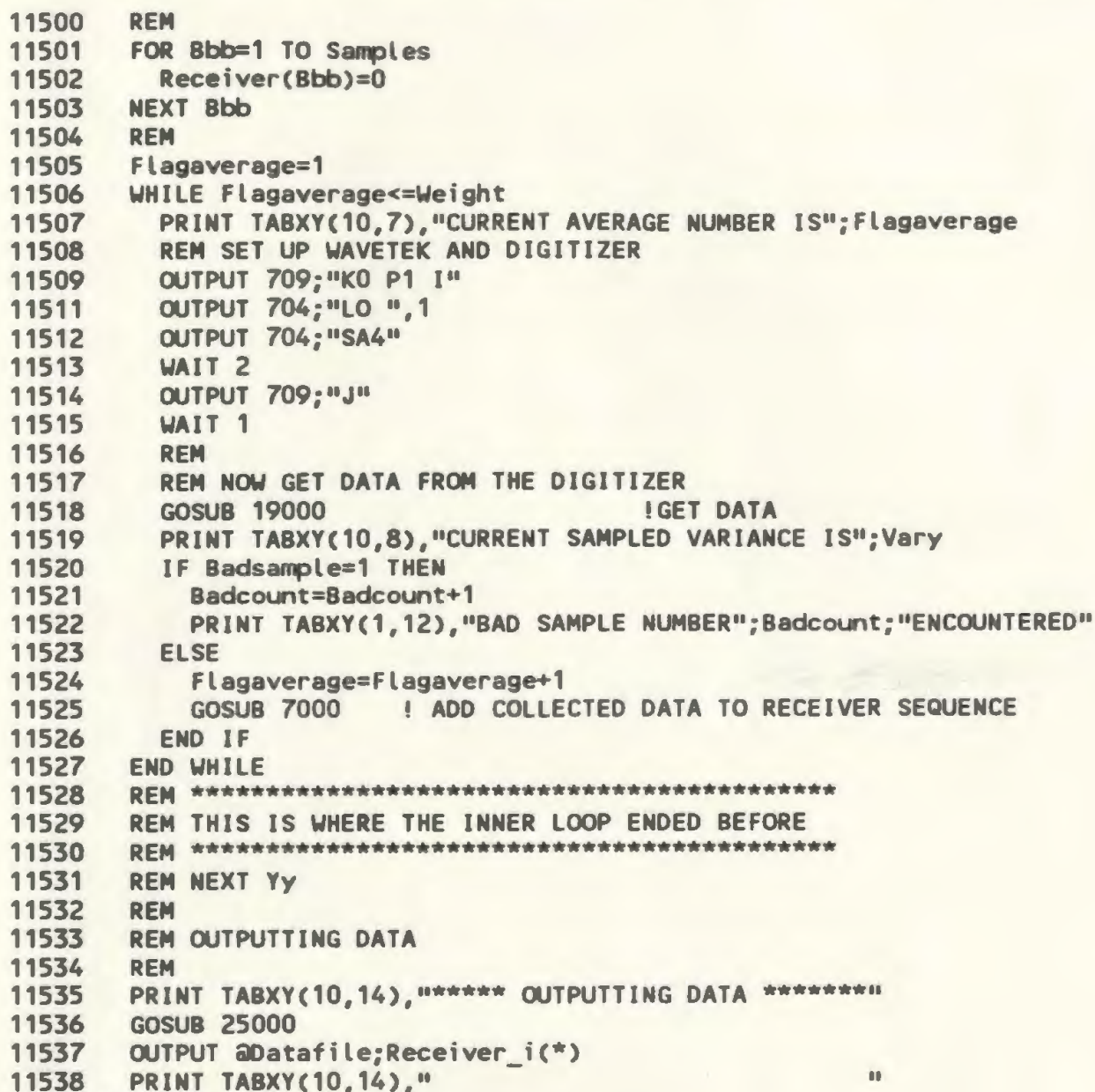

11542 NEXT $X X$

11543 REM

11544 REM THIS IS WHERE THE OUTER LOOP ENDED

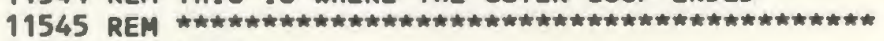

11546 REM NEXT Rr

$11549 X=0$

$11550 Y=0$

$11551 Z=0$

11552 GOSUB 1000 ! RESET THE AXIS

11553 GOSUB 9000 ! TURN OFF WAVETEK

11554 RETURN 0

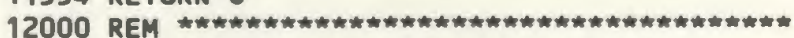

12005 REM OUTPUTS A CONTINUOUS HAVEFOR*

12010 REM

12015 REM

12020 OUTPUT 709;"A1 80 DO Y4000 I"

12025 CLEAR SCREEN

12030 INPUT "INPUT THE FREQUENCY IN HZ (10mHZ - 12MHZ)",sfreq

12035 If sfreq<1.0E-2 OR Sfreq $1.2 E+7$ THEN

12040 PRINT

12045 PRINT "WRONG RESPONSE, TRY ARAIN"

12050 PRINT

12055 GOTO 12030

12060 END IF

12065 PRINT "INPUT THE FUNCTION"

12070 PRINT " O) SINE"

12075 PRINT " 1) TRIANGLE"

12080 PRINT " 2) SQUARE"

12085 PRINT

12090 INPUT sfunc

12095 IF sfunc $>2$ OR Sfunc<0 THEN 


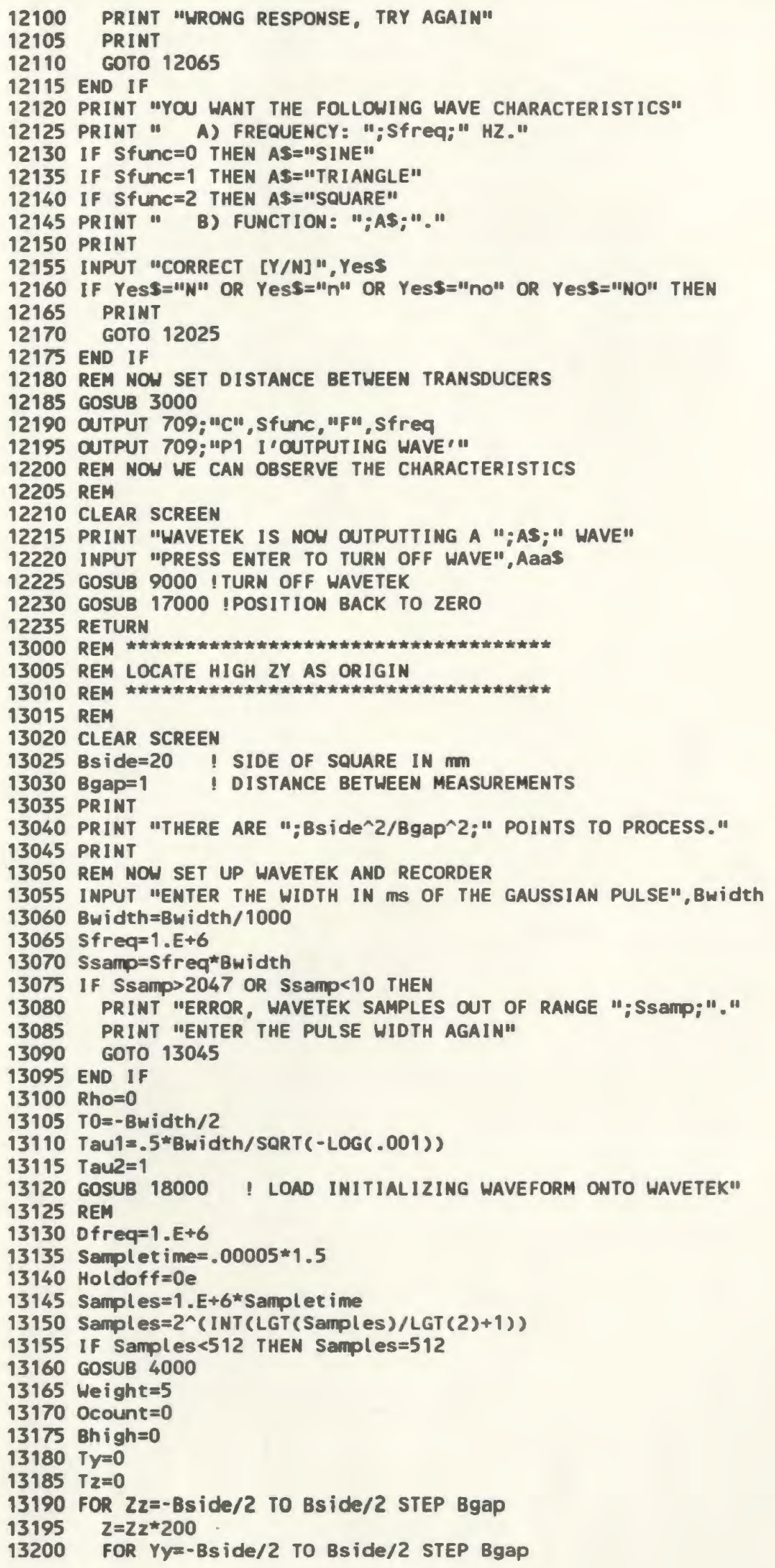




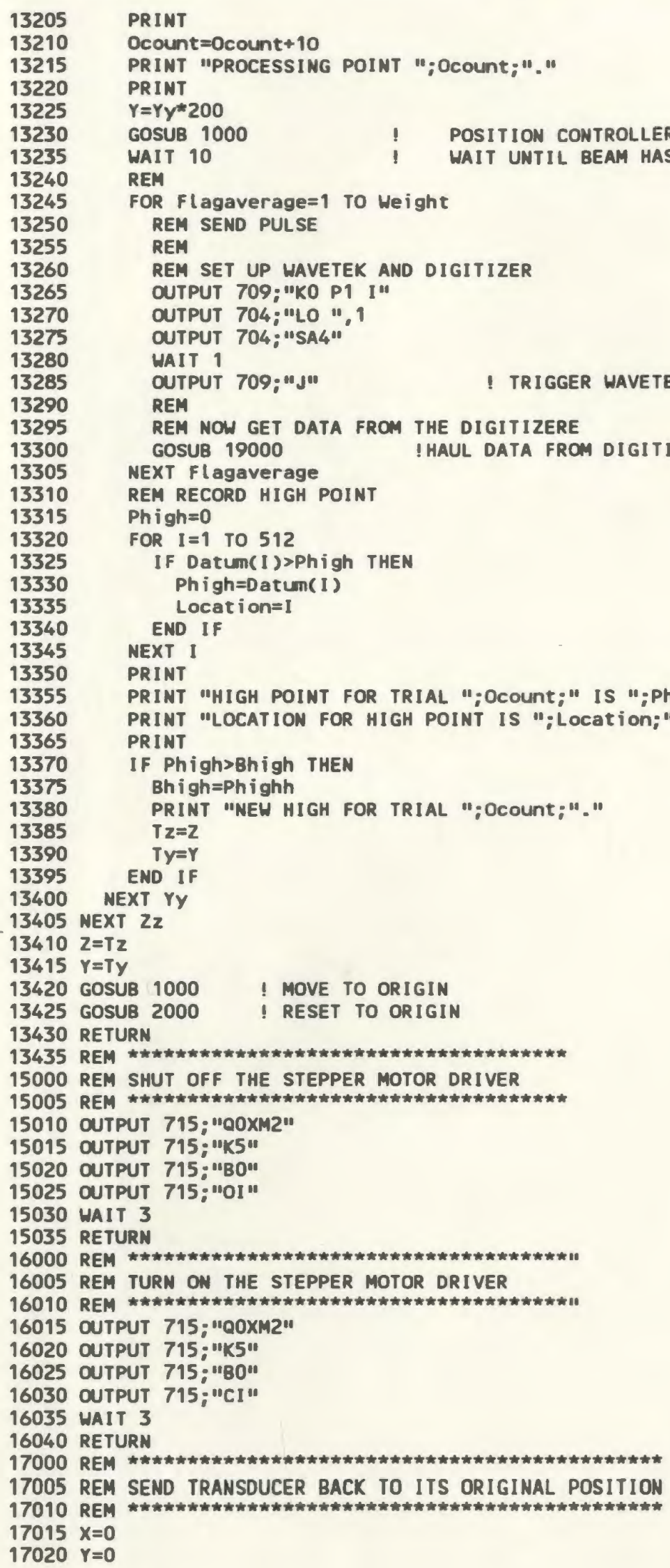




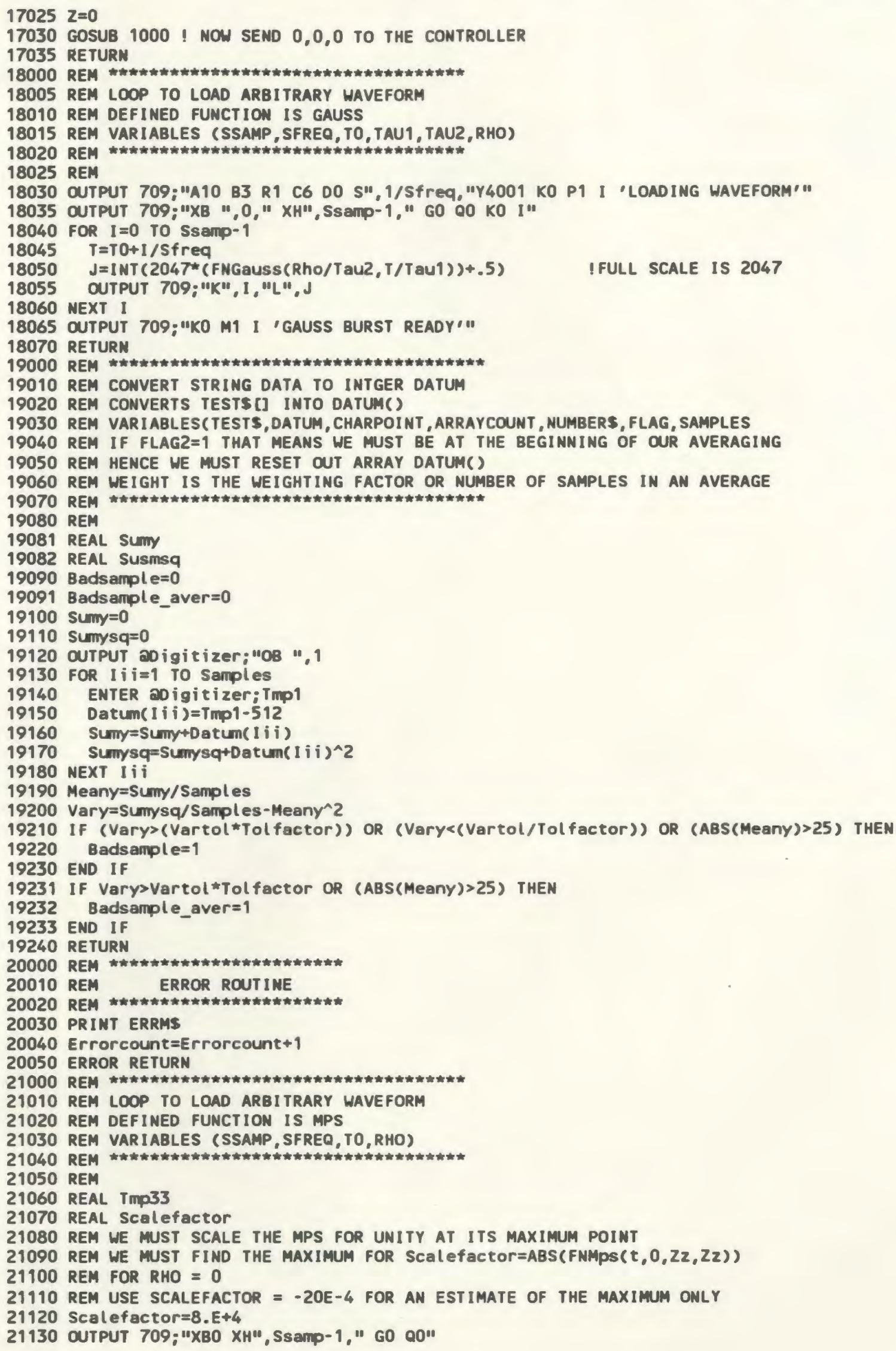


21140 OUTPUT 709;"A10 B3 R1 C6 DO S",1/Sfreq,"Y4001 KO P1 I "LOADING WAVEFORM""

21150 FOR I $\mathrm{i} i=0$ TO Ssamp-1

$21160 T=($ I i i-Ssamp/2)/Sfreq

21170 Tmp33=FNMps (T, Srho)e

$21180 \mathrm{~J}=$ INT (2047* (Tmp33/Scalefactor) +.5)

21190 OUTPUT 709: "K", I I i, "L", J

21200 NEXT Ii

21210 OUTPUT 709;"KO M1 I 'MPS BURST READY""

21220 RETURN 7

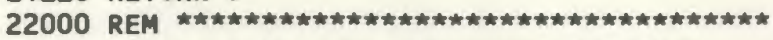

22010 REM LOOP TO LOAD ARBITRARY WAVEFORM

22020 REM DEFINED FUNCTION IS SINC

22030 REM VARIABLES (SSAMP, SFREQ, TAU, FO)

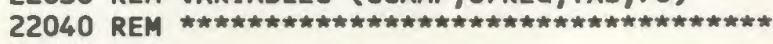

22050 REM

22060 OUTPUT 709; "XBO XH", SsaMP-1," GO QO I"

22070 OUTPUT 709;"A10 B3 R1 C6 DO S", 1/Sfreq,"Y4001 KO P1 I 'LOADING WAVEFORM""

22080 FOR $I=0$ TO Ssamp- 1

$22090 \quad T=I /$ Sfreq

22100 Tmp33=FNSinc $(T, F O, T$ au $)$

$22110 \quad J=I N T(2047 *(T m p 33)+.5)$

22120 OUTPUT 709; "K", I, "L",J

IFULL SCALE IS 2047

22130 NEXT I

22140 OUTPUT 709;"KO M1 I 'SINC BURST READY'"

22150 RETURN

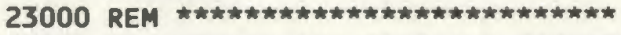

23010 REM CHANGE THE SAMPLING FREQUENCY

23020 REM OF DIGITIZER

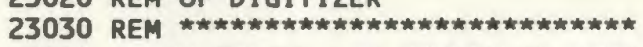

23040 INPUT "ENTER THE AMOUNT OF TIME YOU WISH TO SAMPLE IN ms", Sampletime

23050 Samplet ime=Samplet $\mathrm{ime} / 1000$

23060 Shortsamples=Samplet ime ${ }^{\star} D$ freq

23070 Samples $=2^{\wedge}$ ( INT (LGT (Shortsamples)/LGT (2)+1))

23080 If Samples $<512$ THEN Samples $=512$ )

23090 IF Samples>16900 THEN

23100 PRINT "ERROR, TOO MANY SAMPLES."

23110 PRINT "TRY A SMALLER SAMPLE TIME"

23120 PRINT

23130 GOTO 23040

23140 END IF

23150 RETURN

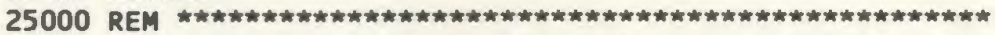

25010 REM * CONVERT RECEIVER REAL DATA INTO INTEGERS $\star * *$

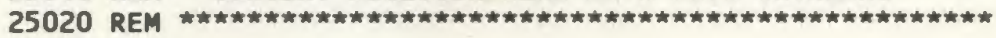

25030 REM

25040 FOR C $c c=1$ TO Samples

25050 Receiver_i(CCC)=INT (Integer_scale*Receiver(Ccc)+.5)

25060 NEXT CCC

25070 RETURN

29000 ENDU

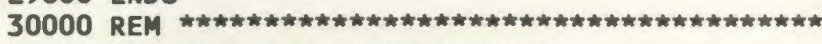

30010 REM DEFINE THE GAUSS PULSE

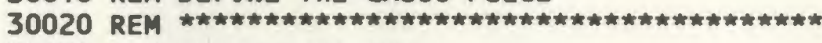

30030 DEF FNGaUSS(R1,T1)

30040 Arbit $=-\operatorname{EXP}\left(-T 1^{\wedge} 2\right) \star \operatorname{EXP}\left(-R 1^{\wedge} 2\right)$

30050 RETURN Arbit

30060 FNEND

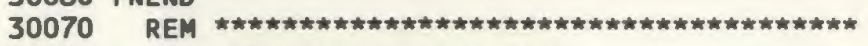

30080 REM DEFINE THE MPS PULSE

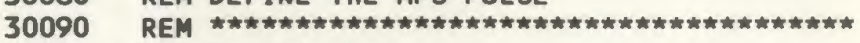

30100 DEF FNMPS(T1, Rho)

30110 REM T1 IS THE TIME ABSOLUTELY

30120 REM RHO IS THE SOURCE ( $\left.x, \wedge 2+y^{\prime} \wedge 2\right)$

30130 REM OZ IS THE OBSERVATION $Z$ CO-ORDINATE

30140 REM Rnn IS SORT $\left(\left(x-x^{\prime}\right)^{\wedge} 2+\left(y^{-} y^{\prime}\right)^{\wedge} 2+\left(z-z^{\prime}\right)^{\wedge} 2\right)$

30150 COMPLEX EYe

30160 REAL Arbit, A, B, Alph, C, 20,Beta, Zmct, Rphi)

30170 COMPLEX S,Dzs,Dzf,Dts,Dtf, F,Ph im

$30180 \quad$ EYe $=C M P L X(0,1)$ 


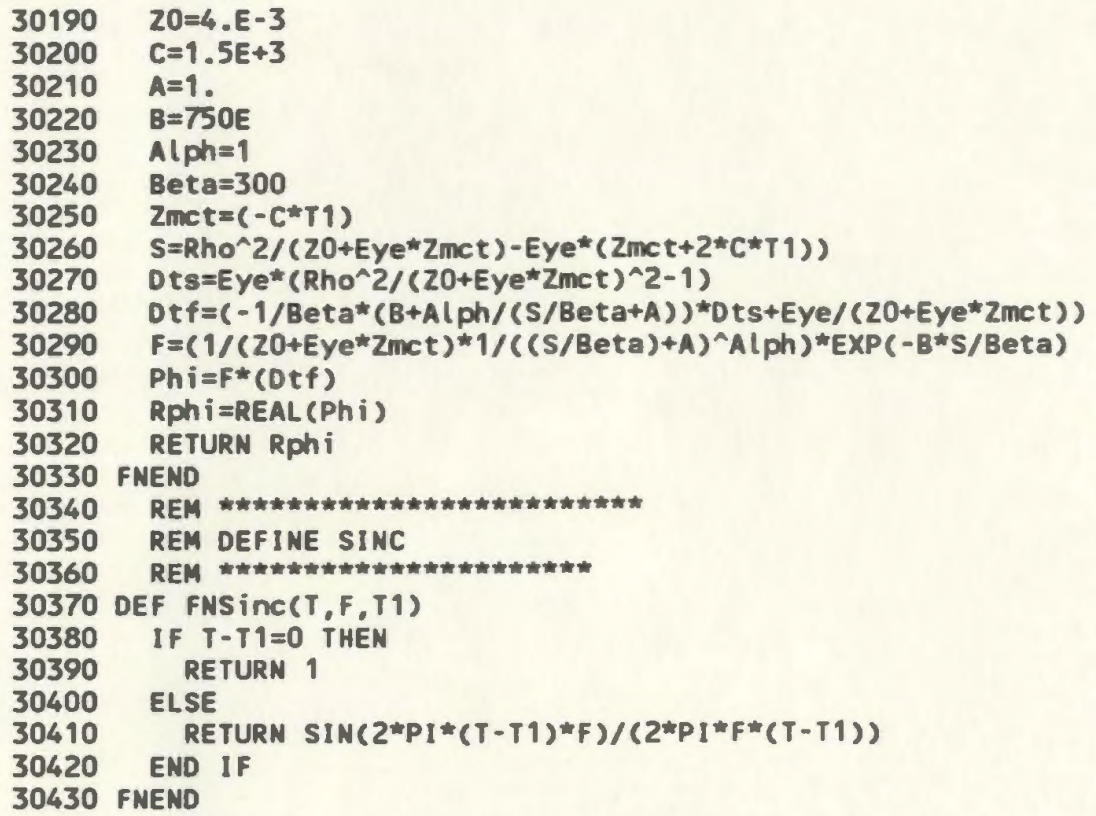




\section{Appendix B: Matlab Algorithm for Extraction of Sphere Radius}

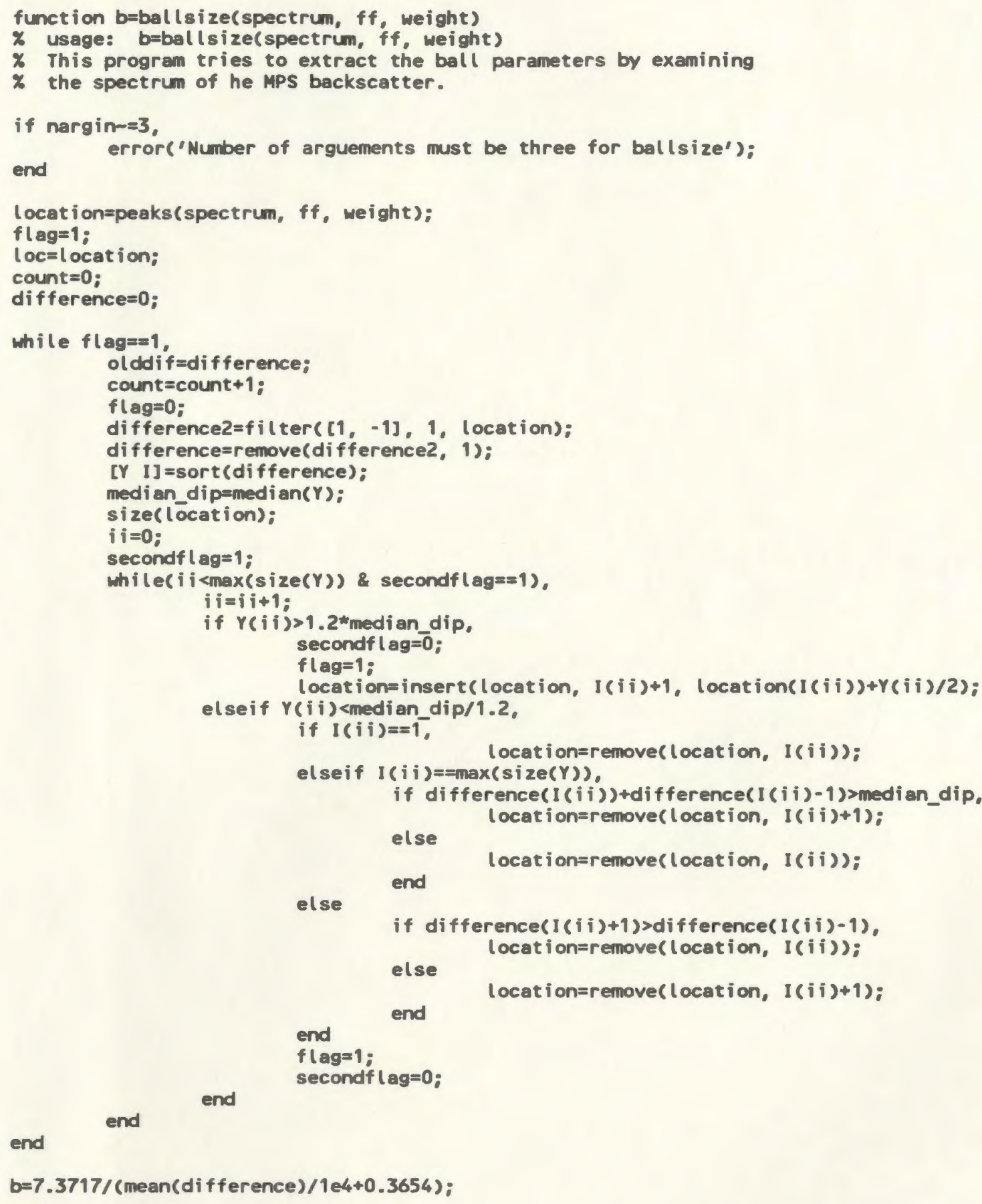




\section{Appendix C \\ Measured Backscattered Spectra}

Plots not included in text 


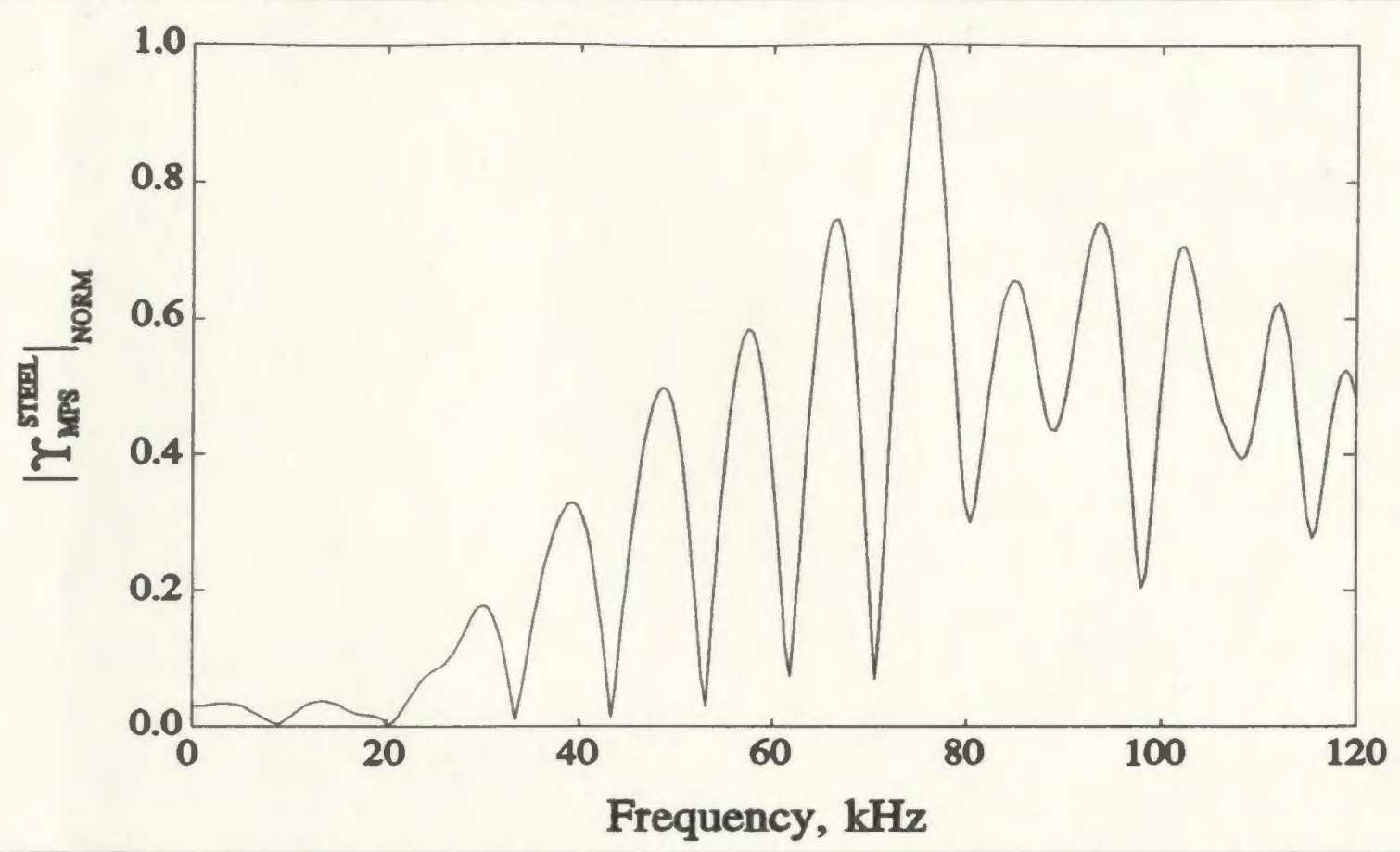

Figure C.1: $\left|\Upsilon_{\text {MPs }}\right|$ for a $60 \mathrm{~mm}$ radius steel sphere at $r=250 \mathrm{~mm}$ from the array.

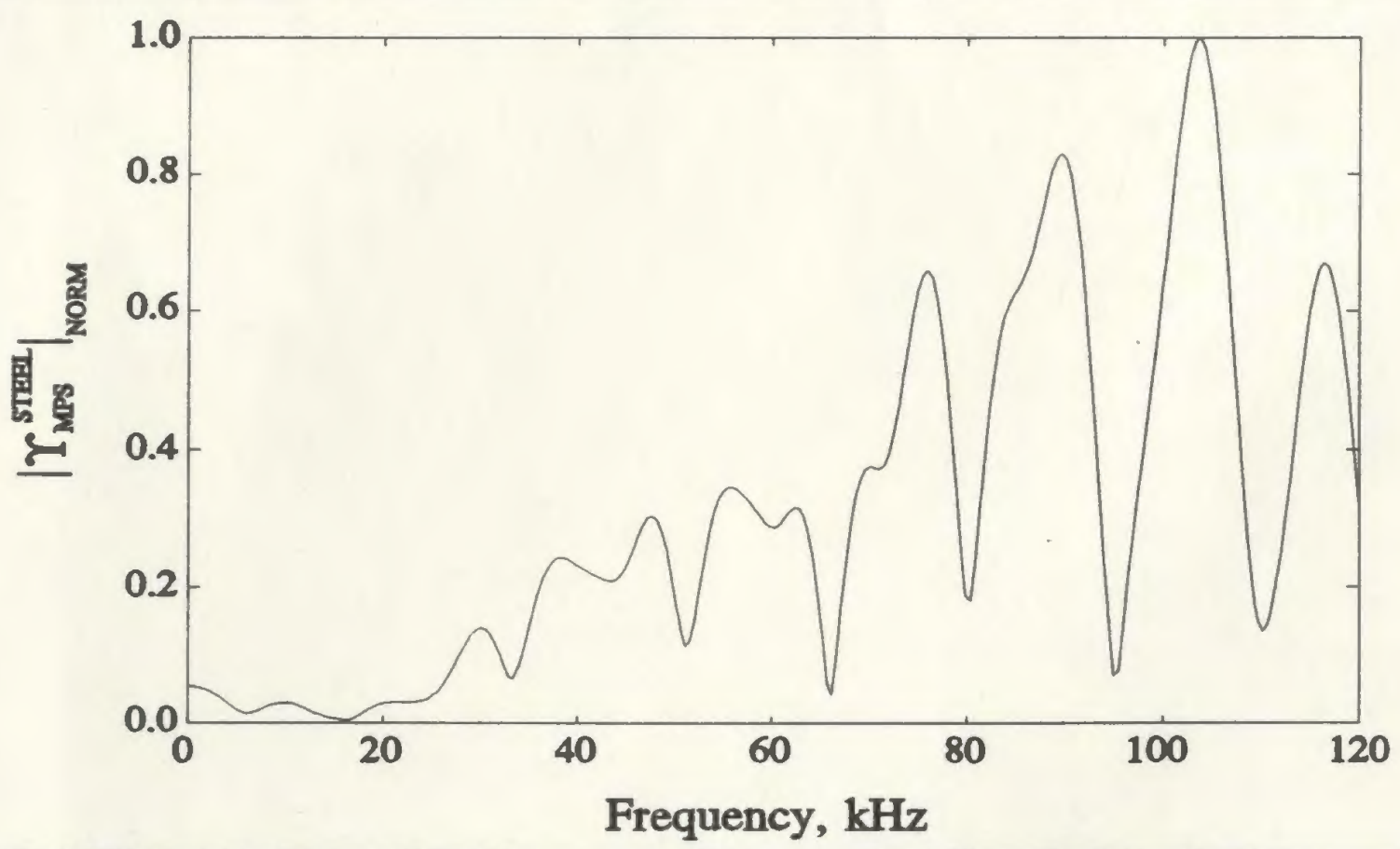

Figure C.2: $\left|\Upsilon_{\text {MPS }}\right|$ for a $40 \mathrm{~mm}$ radius steel sphere at $r=250 \mathrm{~mm}$ from the array. 


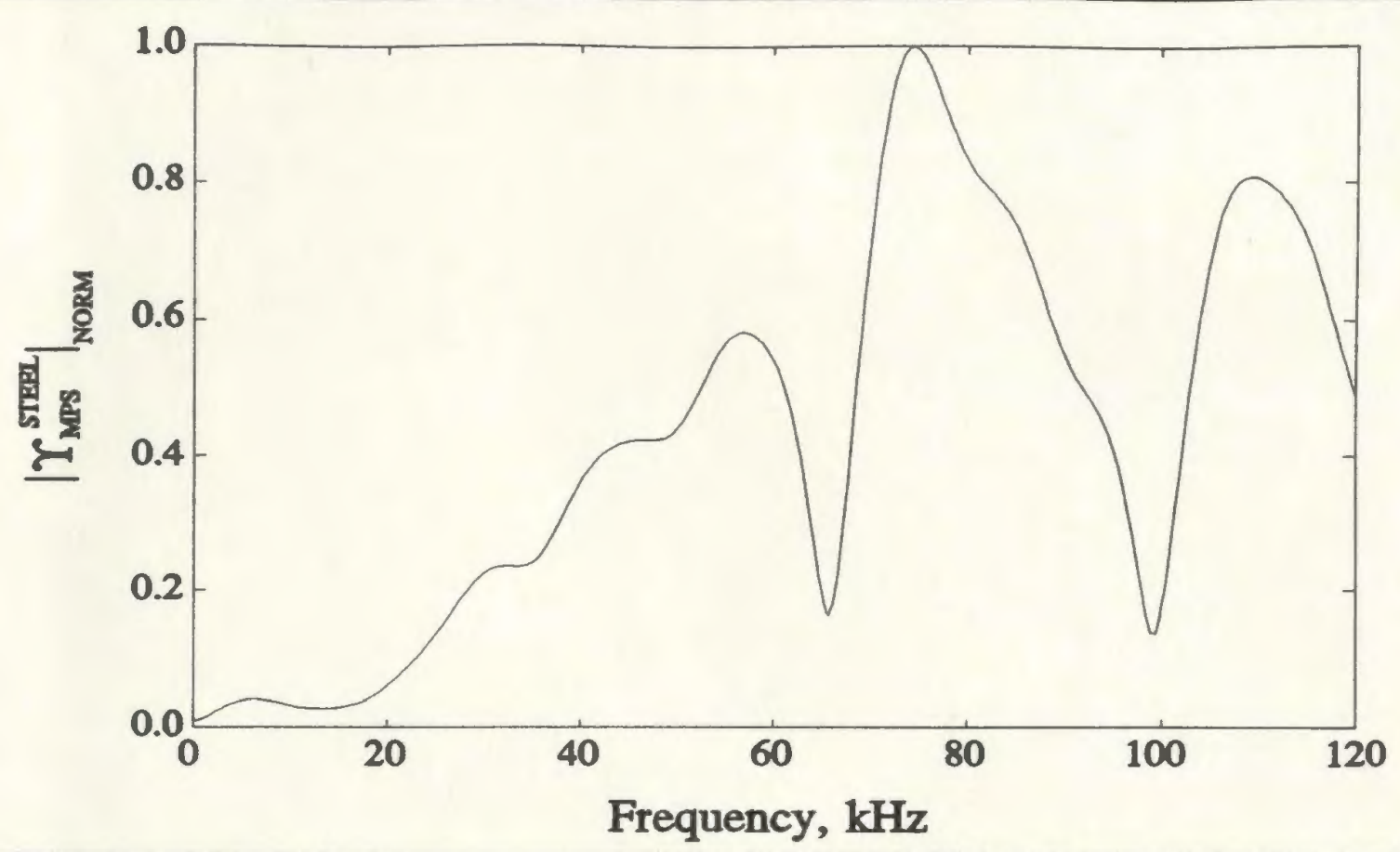

Figure C.3: $\left|\Upsilon_{\text {MPS }}\right|$ for a $30 \mathrm{~mm}$ radius steel sphere at $r=250 \mathrm{~mm}$ from the array.

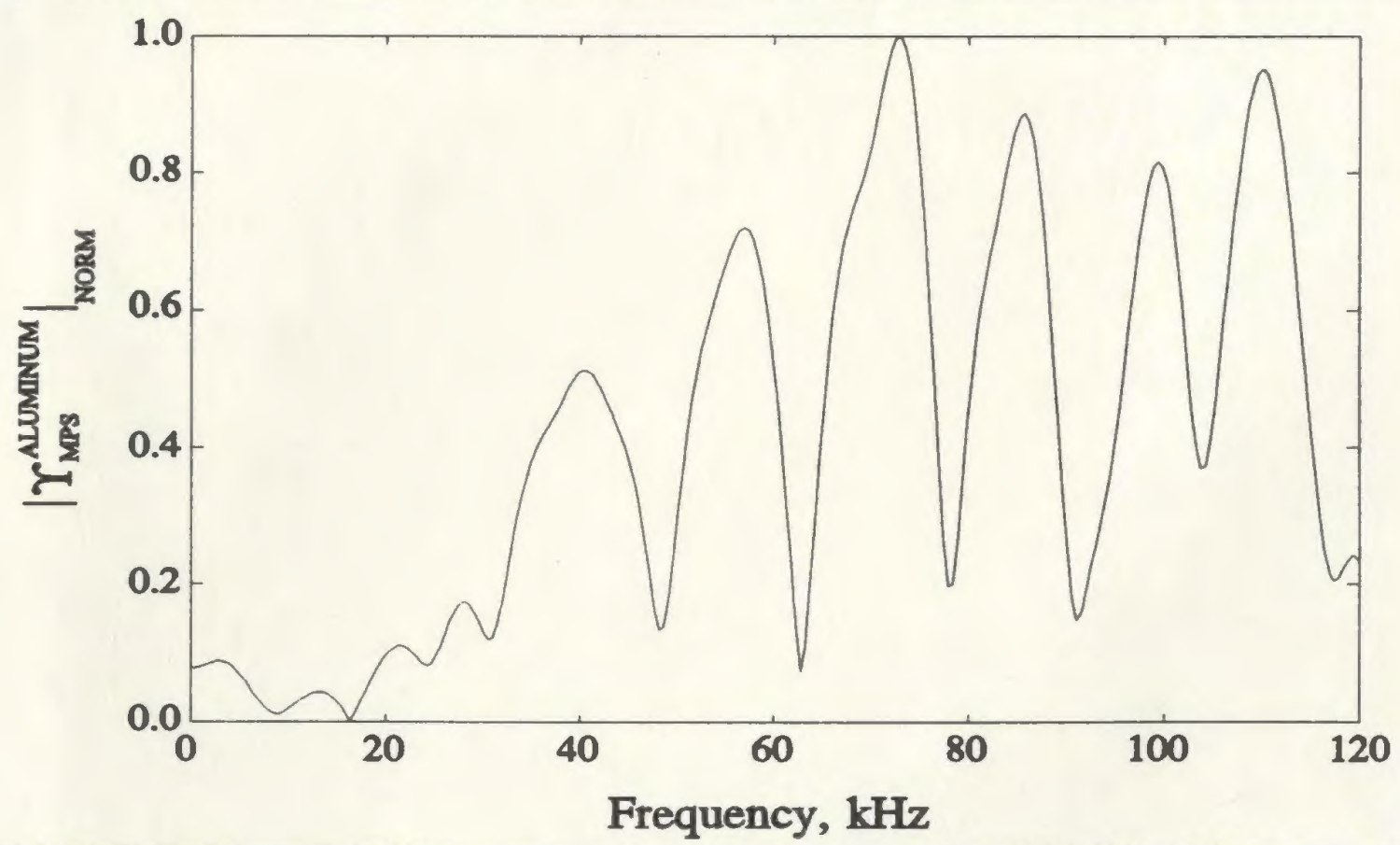

Figure C.4: $\left|\Upsilon_{\text {MPS }}\right|$ for a $40 \mathrm{~mm}$ radius aluminum sphere at $r=250 \mathrm{~mm}$ from the array. 


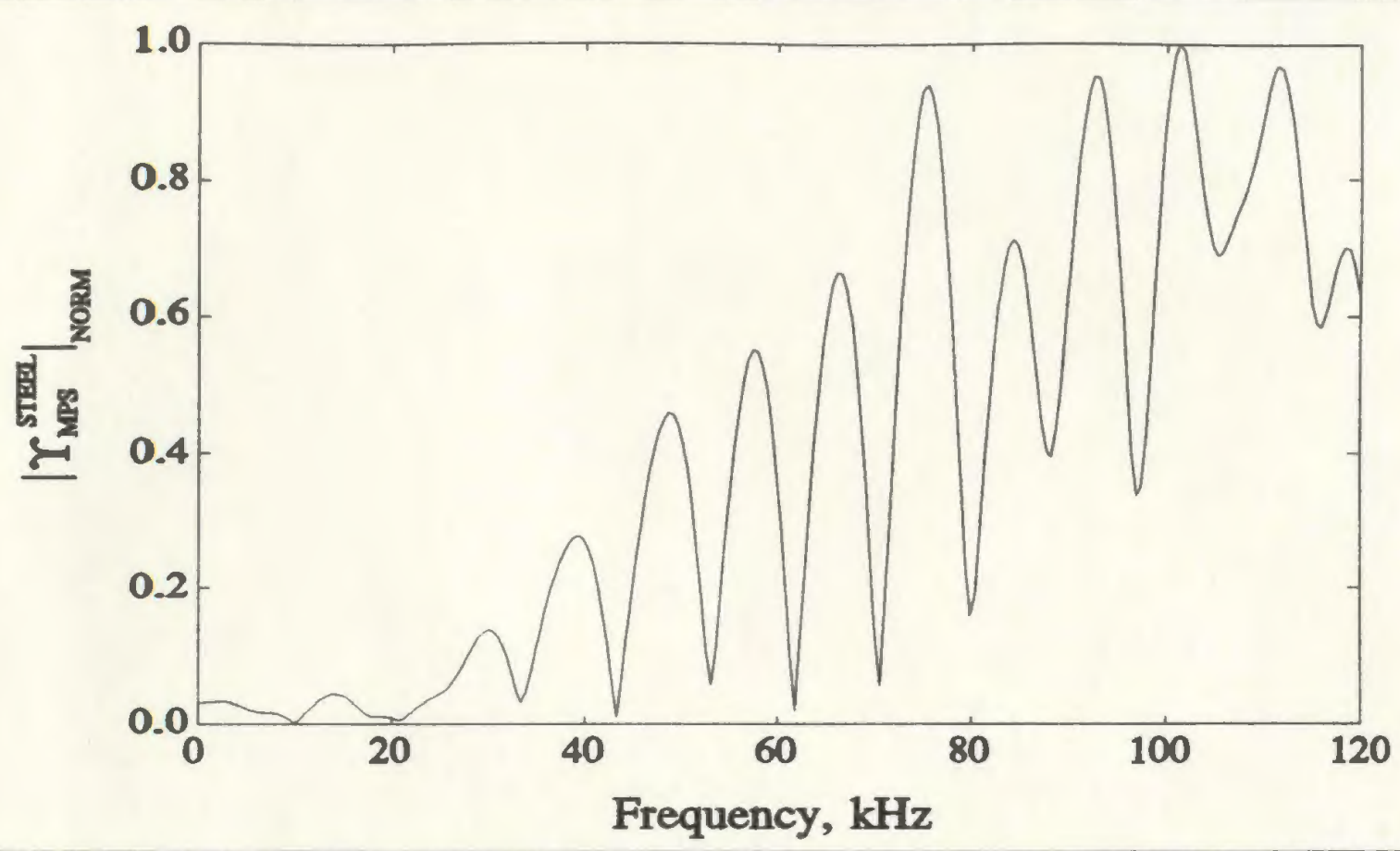

Figure C.5: $\left|\Upsilon_{\text {MPS }}\right|$ for a $60 \mathrm{~mm}$ radius steel sphere at $r=350 \mathrm{~mm}$ from the array.

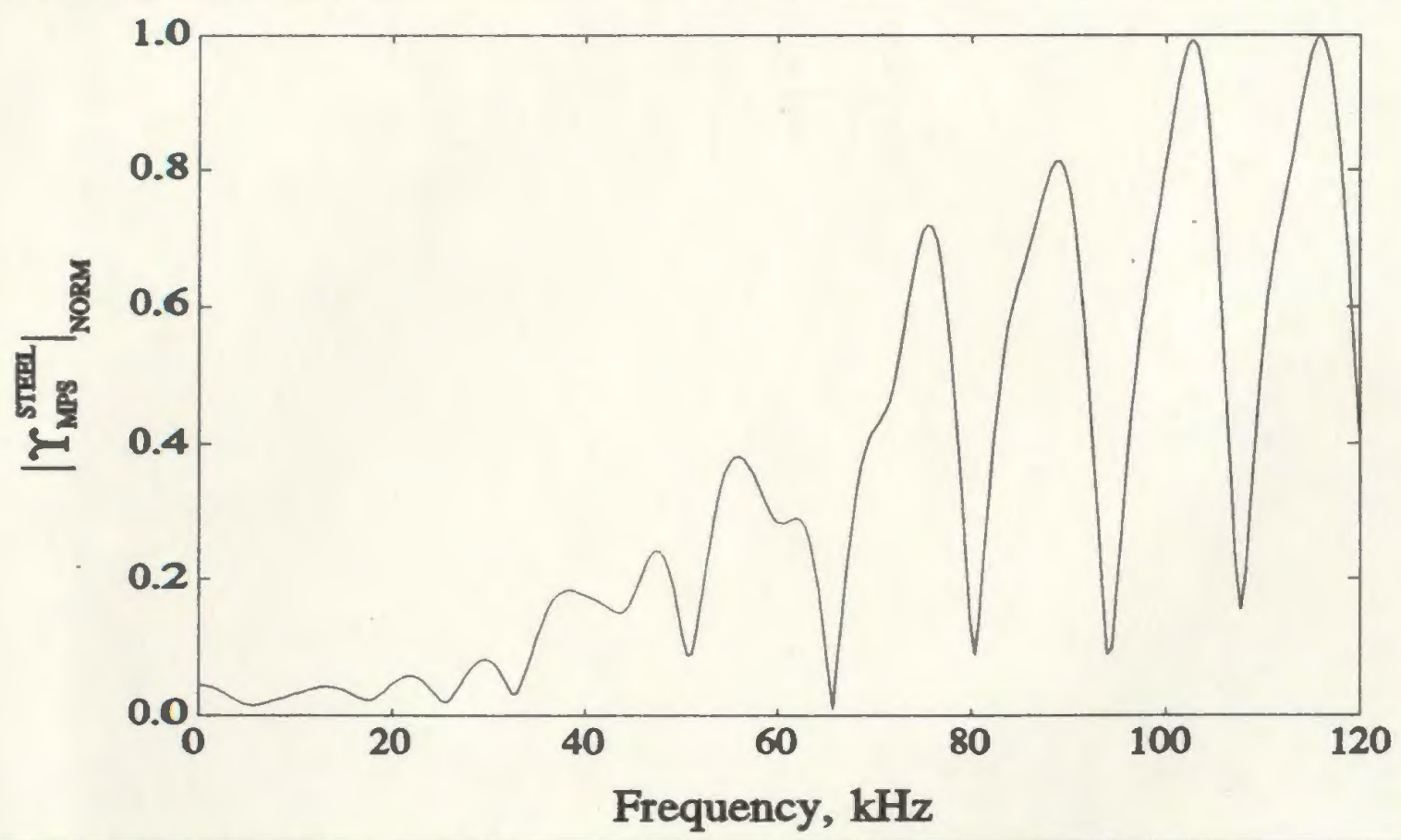

Figure C.6: $\left|\Upsilon_{\text {MPS }}\right|$ for a $40 \mathrm{~mm}$ radius steel sphere at $r=350 \mathrm{~mm}$ from the array. 


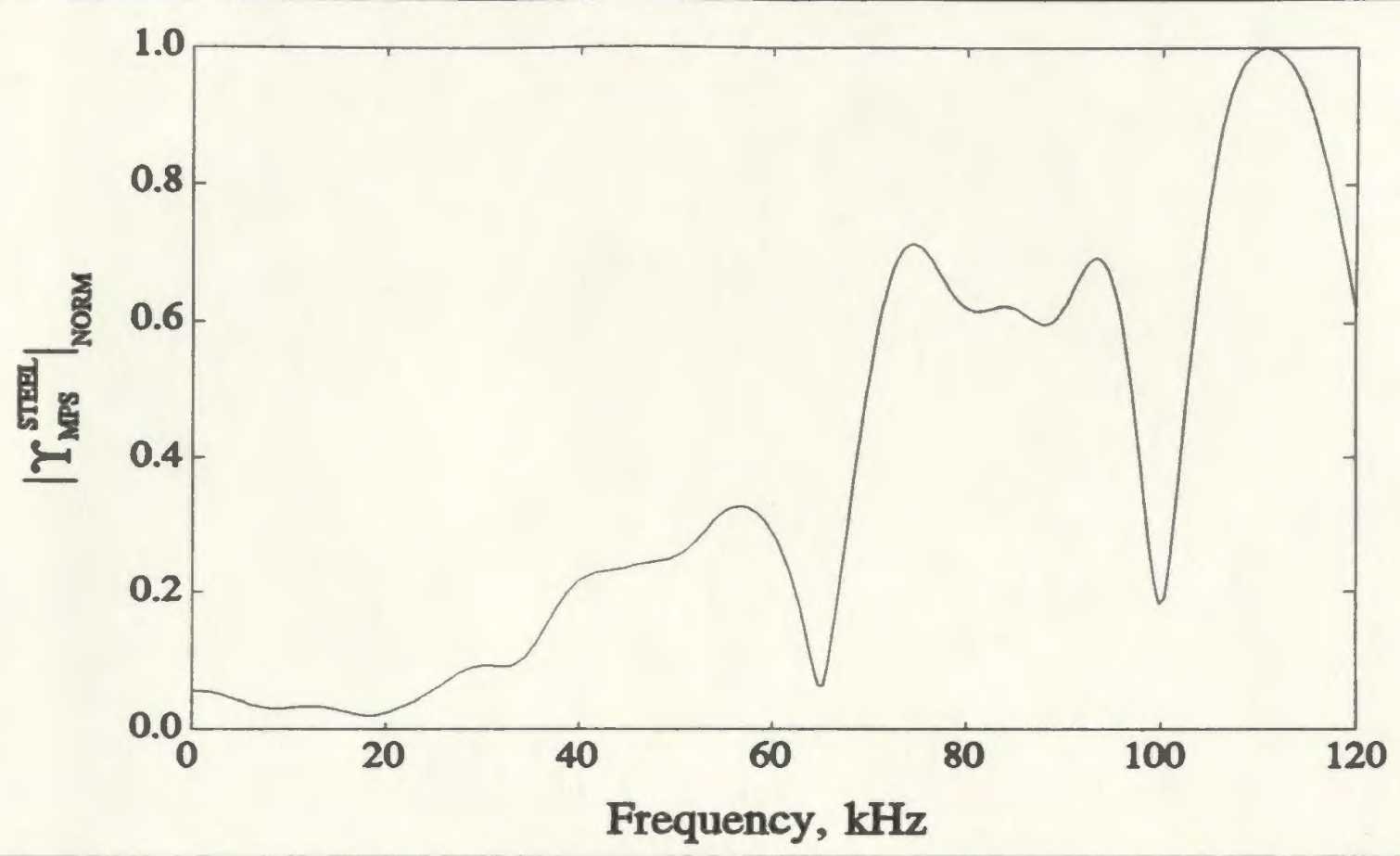

Figure C.7: $\left|\Upsilon_{\text {MPS }}\right|$ for a $30 \mathrm{~mm}$ radius steel sphere at $r=350 \mathrm{~mm}$ from the array.

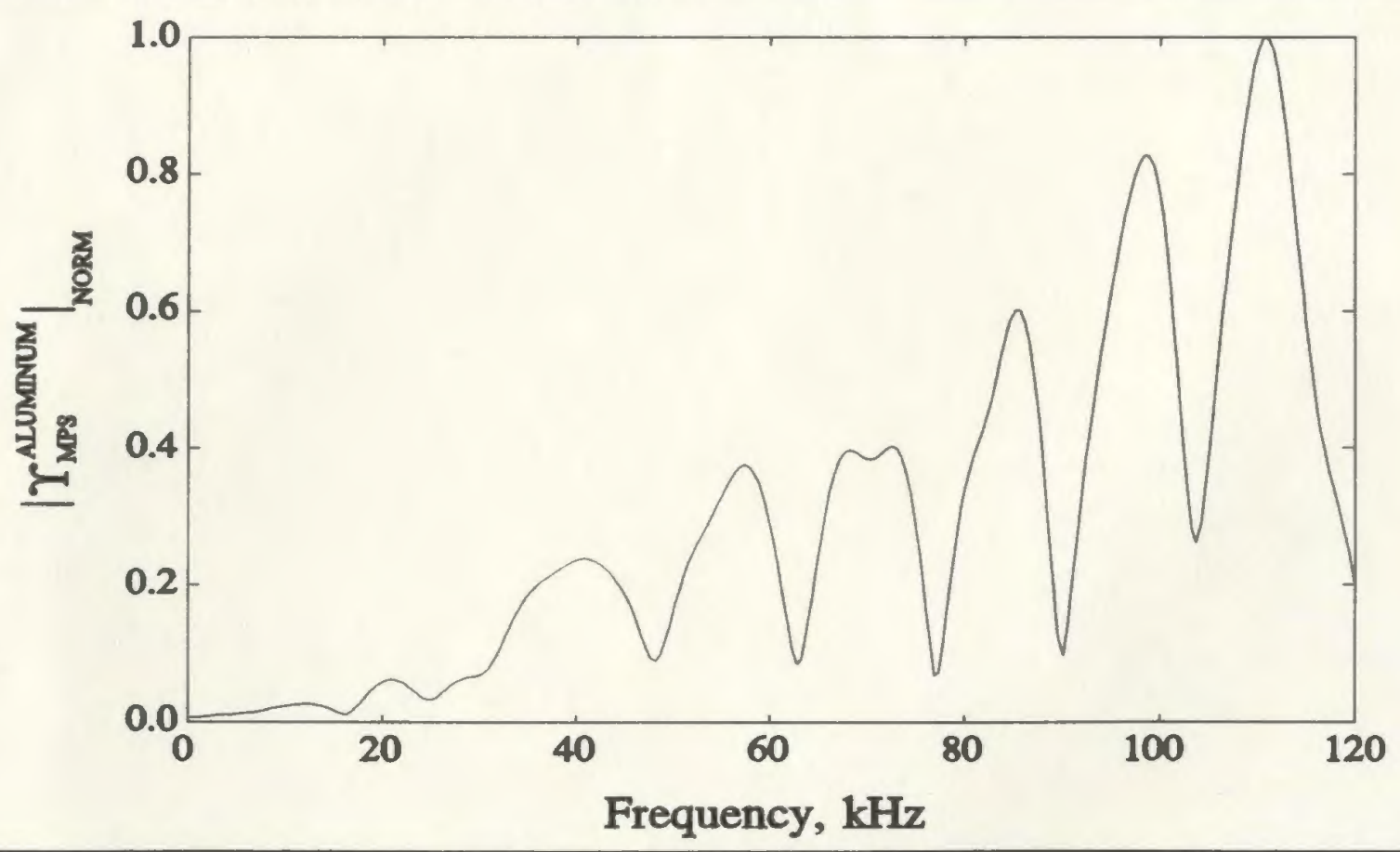

Figure C.8: $\left|\Upsilon_{\text {MPS }}\right|$ for a $40 \mathrm{~mm}$ radius aluminum sphere at $r=350 \mathrm{~mm}$ from the array. 


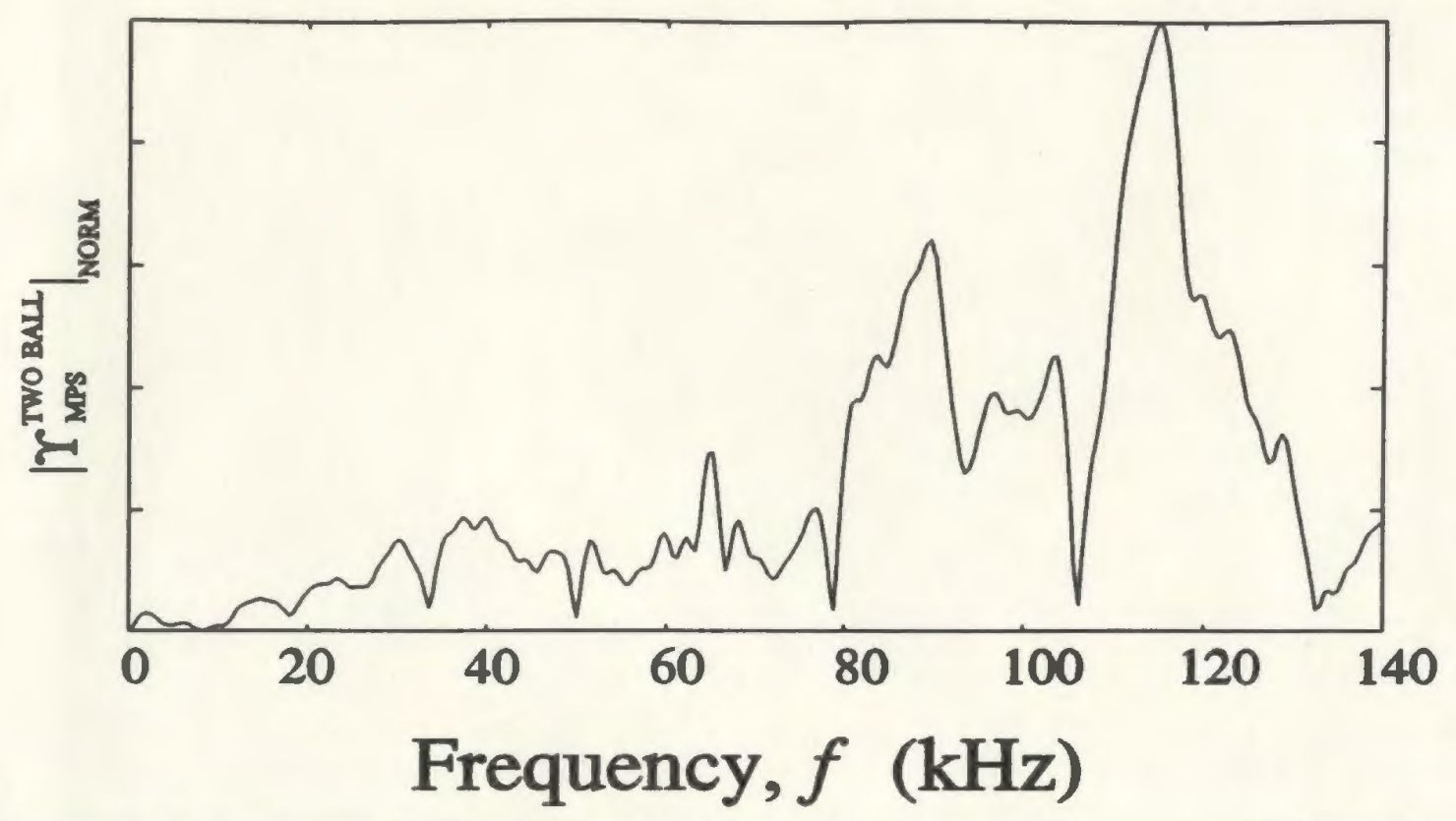

Figure C.9:: Two ball backscatter spectrum for $r=35 \mathrm{~cm}$ and $d=4 \mathrm{~cm}$.

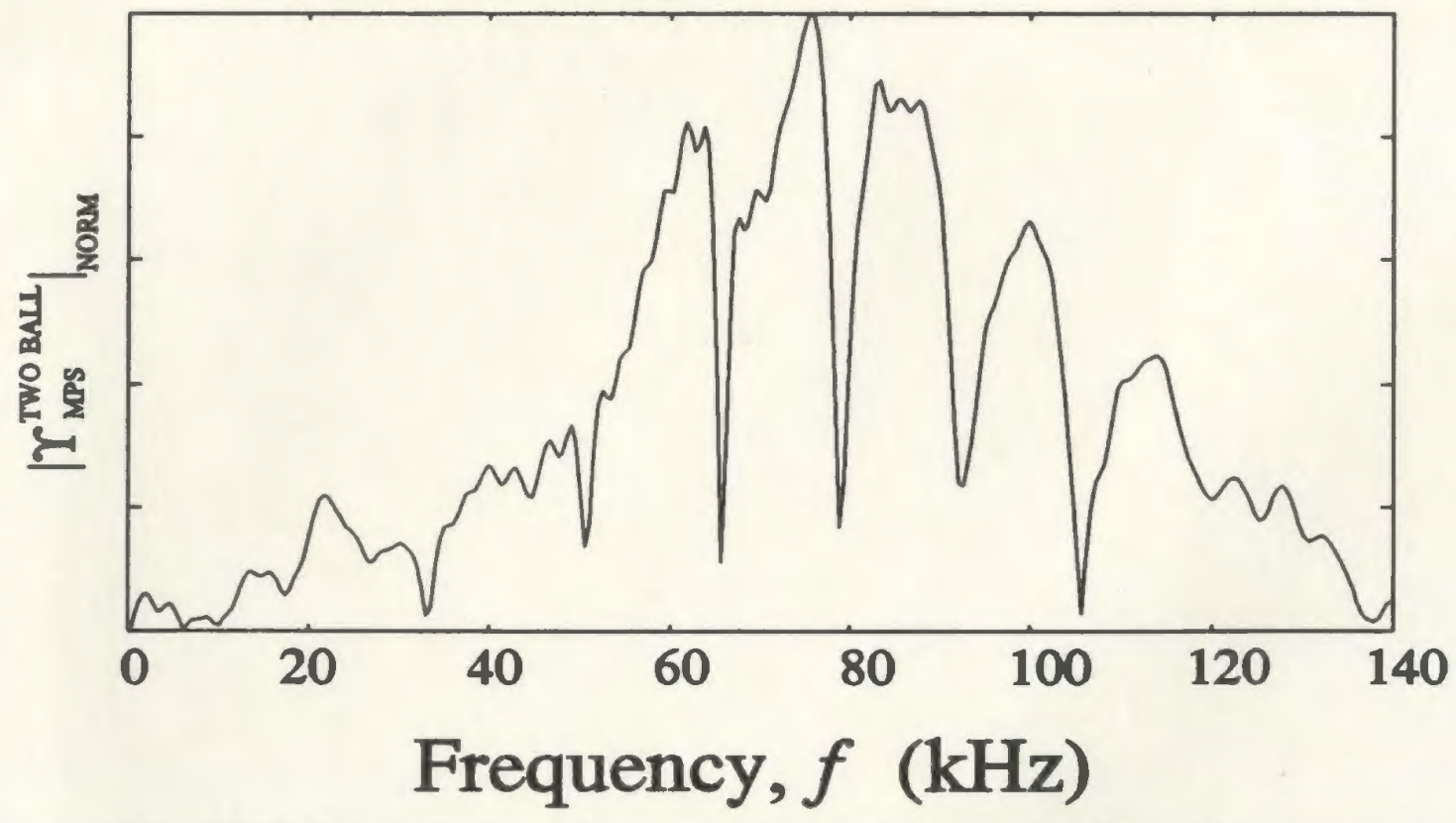

Figure C.10: Two ball backscatter spectrum for $r=35 \mathrm{~cm}$ and $d=8 \mathrm{~cm}$. 


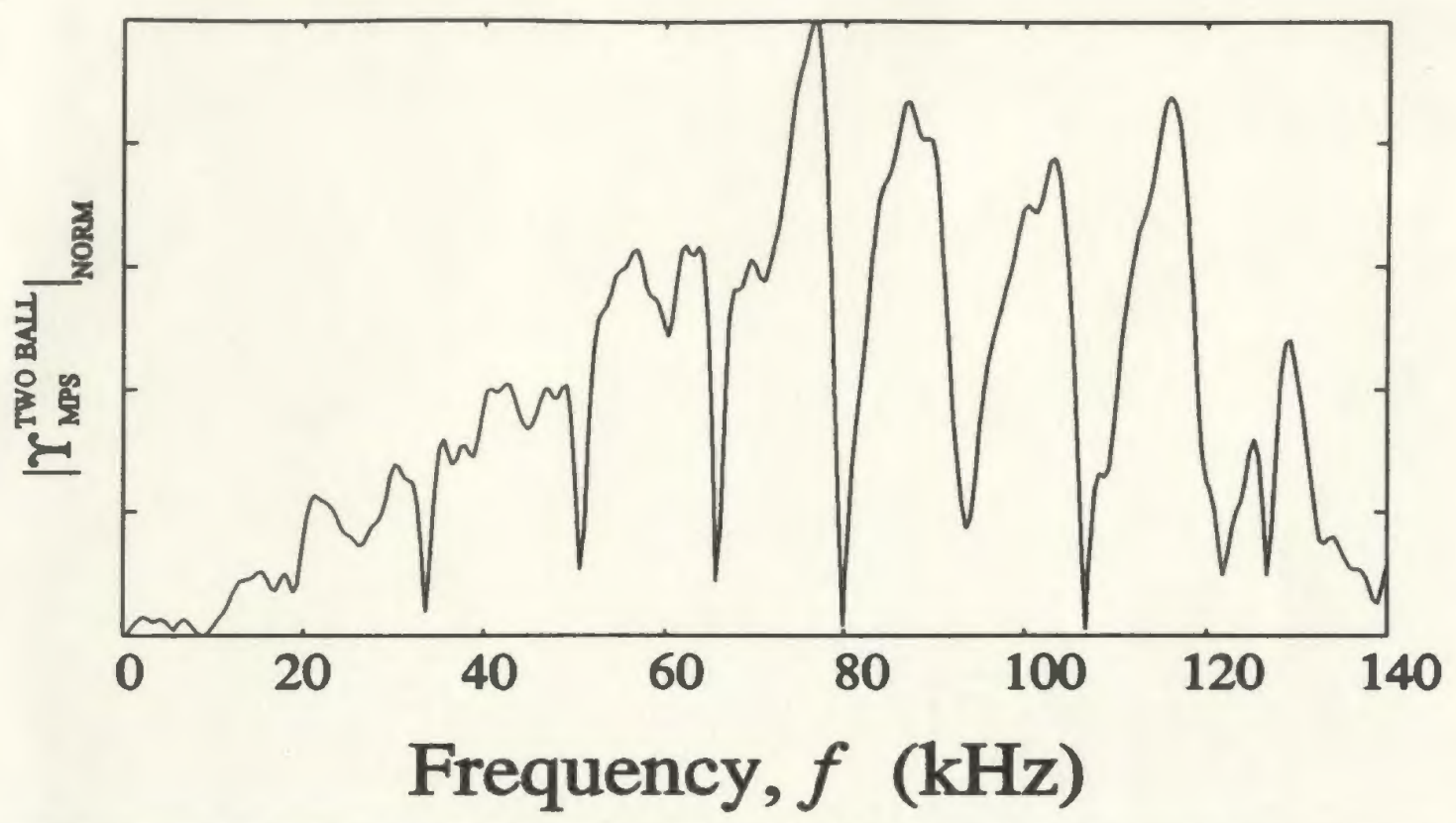

Figure C.11: Two ball backscatter spectrum for $r=35 \mathrm{~cm}$ and $d=14 \mathrm{~cm}$.

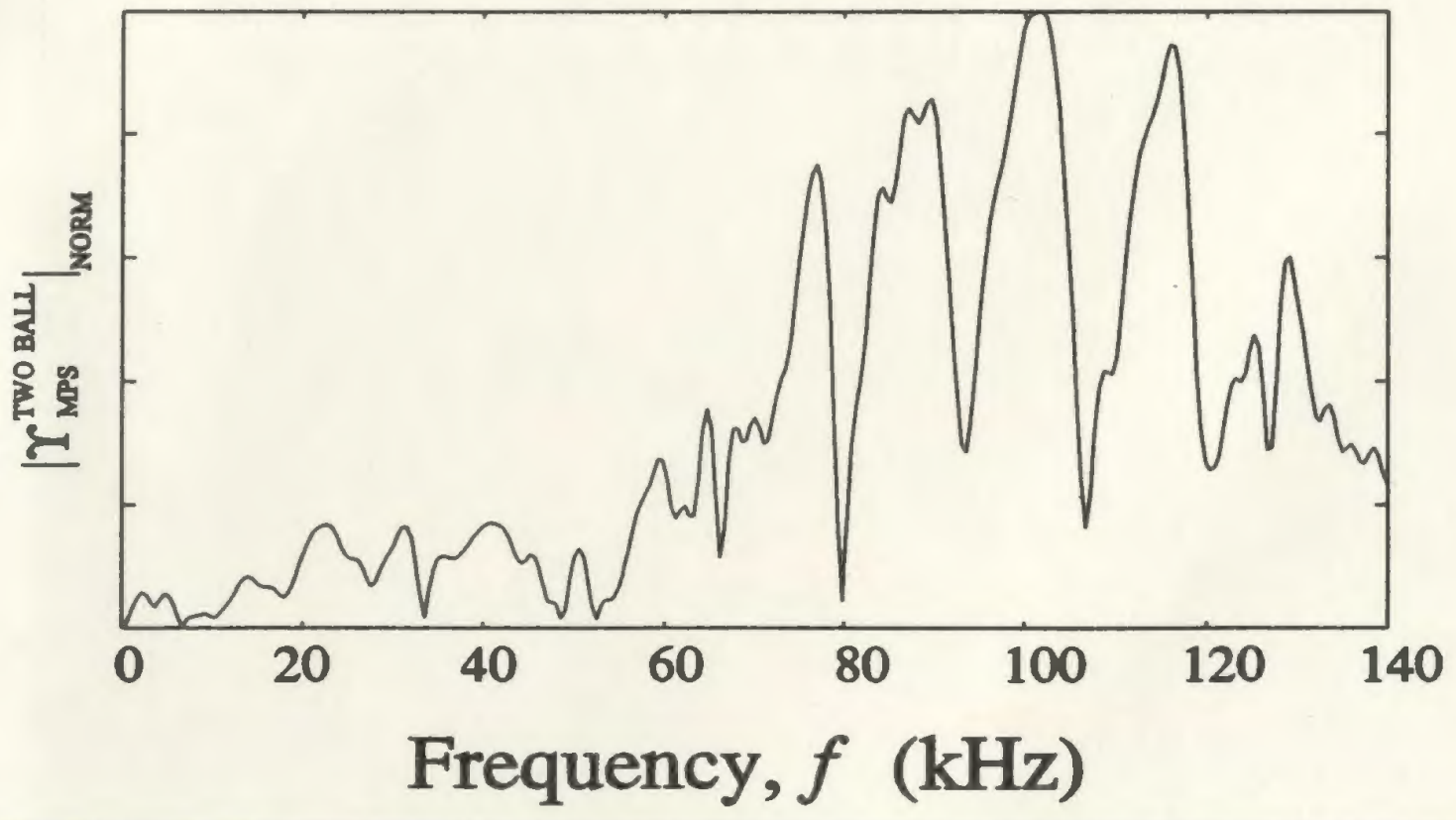

Figure C.12: Two ball backscatter spectrum for $r=35 \mathrm{~cm}$ and $d=20 \mathrm{~cm}$. 


